\title{
Non-target Analyses of Novel Psychoactive Substances; Inherent Variation in GCMS Relative Abundances and Gas Phase Hydrogen Deuterium Exchange Isomer Discrimination
}

Kristin Michelle Kelly

kckelly@mix.wvu.edu

Follow this and additional works at: https://researchrepository.wvu.edu/etd

Part of the Analytical Chemistry Commons

\footnotetext{
Recommended Citation

Kelly, Kristin Michelle, "Non-target Analyses of Novel Psychoactive Substances; Inherent Variation in GCMS Relative Abundances and Gas Phase Hydrogen Deuterium Exchange Isomer Discrimination" (2019). Graduate Theses, Dissertations, and Problem Reports. 3923.

https://researchrepository.wvu.edu/etd/3923

This Dissertation is protected by copyright and/or related rights. It has been brought to you by the The Research Repository @ WVU with permission from the rights-holder(s). You are free to use this Dissertation in any way that is permitted by the copyright and related rights legislation that applies to your use. For other uses you must obtain permission from the rights-holder(s) directly, unless additional rights are indicated by a Creative Commons license in the record and/ or on the work itself. This Dissertation has been accepted for inclusion in WVU Graduate Theses, Dissertations, and Problem Reports collection by an authorized administrator of The Research Repository @ WVU. For more information, please contact researchrepository@mail.wvu.edu.
} 


\title{
Non-target Analyses of Novel Psychoactive Substances; Inherent Variation in GCMS Relative Abundances and Gas Phase Hydrogen Deuterium Exchange Isomer Discrimination
}

\section{Kristin Michelle Kelly}

\author{
Dissertation submitted to the \\ Eberly College of Arts and Sciences \\ at West Virginia University \\ in partial fulfillment of the requirements for the degree of \\ Doctor of Philosophy in Chemistry
}

\author{
Suzanne Bell, Ph.D., Chair \\ Jonathan Boyd, Ph.D. \\ Patrick Callery, Ph.D. \\ Glen Jackson, Ph.D. \\ Stephen Valentine, Ph.D.
}

\section{Eugene Bennett Department of Chemistry}

\section{Morgantown, West Virginia \\ 2019}

Keywords: novel psychoactive substances, gas chromatography, mass spectrometry, mass spectra, relative abundance, variation, ion mobility spectrometry, hydrogen deuterium exchange, isomer discrimination

\section{Copyright 2019 Kristin Michelle Kelly}




\section{Abstract \\ Evaluation of Non-target Analyses of Novel Psychoactive Substances; Inherent Variation in GCMS Relative Abundances and Gas Phase Hydrogen Deuterium Exchange Isomer Discrimination}

\section{Kristin Michelle Kelly}

Novel psychoactive substances (NPSs) are synthetically derived compounds designed to mimic the effects of other illicit drugs. An endless cycle of NPSs continually reach the drug market due to limitations in drug legislation creating two problems: (1) non-availability of standards and (2) multiple isobars indistinguishable even to high resolution mass spectrometry. Gas chromatography-mass spectrometry (GCMS) is the most common instrument used for compound identification in non-targeted seized drug analysis. Compound identification using this technique relies on mass spectra where the percent relative abundances (\%RAs) of all $\mathrm{m} / \mathrm{z}$ values are compared manually or searched against a library database. Lacking standards, laboratories rely on published GCMS spectra to postulate compound identification. The ability to make such identifications provides vital investigative data and may help uncover new structural variants. However, central to such identifications is an understanding of how much spectral patterns vary across laboratories; the assumption that variation is minimized through the use of a standard tuning compound perfluorotributylphosphine (PFTBA) has yet to be rigorously tested and demonstrated. The first focus of this research was the often-unappreciated aspect to any mass spectral comparison or search- the inherent variation in RAs.

In the first study, the variation of mass spectral fragmentation patterns characterized by $\%$ RA was evaluated using three GCMS instruments and 16 NPS compounds. The variation of retention indices was also studied. Retention indices showed $<0.35 \%$ variation across all systems; mass spectral data showed much greater variation across all compounds and systems. Tuning frequency was one factor correlated with a small, but significant, effect on the variance. A t-test comparison of mean ion \%RA did not clearly identify general patterns. The identity of the base peak changed for three compounds. Alternating base peaks on a single instrument, i.e. one instrument produced multiple base peak ions over the course of the study, generated a ripple effect, where variation across all \%RAs in the effected spectra increased. However, alternating base peaks between instrument, i.e. each instrument produced a single base peak but the ion identity was not necessarily the same, did not produce these ripple effects. The variance in \%RA caused by alternating base peaks has the potential to skew compound identification during nontargeted analyses, which are common in a forensic drug chemistry setting.

Decades ago, the Environmental Protection Agency (EPA) established tune check compounds as a means to control systematic variation across vendor platforms, which was the focus of the second study. The goal of the second study was to evaluate whether the decafluorotriphenylphosphine (DFTPP) criteria, as defined by the EPA, could be used to reduce the RA variation in mass spectra produced by NPSs. Instruments from two vendors were used to analyze 6 NPSs: 4 cannabinoids and 2 cathinones. Each NPS was analyzed 100 times per instrument; 10 replicates per tune repeated under 10 different tunes. Results showed that passing a DFTPP tune check was not correlated with reduced RA variation. Tuning algorithm differences 
between vendors did impact the \%RA variance, but the frequency of instrument tuning was found to be the most critical factor for controlling RA variation. The results of this work suggest that forensic laboratories should develop quantitative metrics to evaluate autotuning results and define how these metrics will be used to dictate maintenance. This practice, coupled with tuning before each analytical batch, will reduce the variation of \% RA values as much as practicable.

Because GCMS usually cannot distinguish constitutional isomers, the second focus of this research was to develop a novel methodology for the NPS isomer discrimination. Ion mobility spectrometry-mass spectrometry (IMS-MS) is used as a non-confirmatory screening technique for illicit substances in high traffic areas. Through the years, advancements in instrumentation have improved detection limits, increased resolving power, and allowed for unique instrument modifications which improve structural inferences. Gas-phase hydrogen deuterium exchange (HDX) is one such modification technique. HDX-IMS-MS has been used primarily for proteomic and metabolomic applications, but it is also effective in discriminating of small biomolecules (glycan linkages).

For the final study, NPSs containing labile heteroatom hydrogens were evaluated for HDX reactivity in the presence of either deuterated water $\left(\mathrm{D}_{2} \mathrm{O}\right)$ or ammonia $\left(\mathrm{ND}_{3}\right)$ within the drift tube. An initial evaluation of exchange propensity was performed for six NPSs. Five of the initial NPSs exchanged in the presence of $\mathrm{ND}_{3}$, while only one NPS (benzyl piperazine) exchanged with $\mathrm{D}_{2} \mathrm{O}$. The exchange mechanism of $\mathrm{D}_{2} \mathrm{O}$ requires stabilization with a nearby charged site; the diamine ring of benzyl piperazine provided this charge site at a fixed length. Three disubstituted benzene isomers (ortho-, meta-, and para-) containing the diamine ring structure and a fluorine atom were analyzed to determine if isomer discrimination was possible. These isomers had nearly identical collisional cross sections and isotopic distributions in the drift gas, therefore IMS and MS alone cannot discriminate between the isomers. A t-test of means $(\alpha=0.05)$ showed that discrimination was possible if the exchange data from both reagent gases were included. Molecular dynamics simulations showed that the proximity of the fluorine to the diamine ring hinders the dihedral angle between the benzene ring and the diamine ring. The angle limitation partially accounts the observed exchange differences. One clear limitation of this technique is that the substance must exchange in the presence of both reagent gases. Nevertheless, this technique was shown to be capable of isomer discrimination in non-targeted analyses of NPSs 


\section{Dedication}

Dedicated to my mother and father, your endless support, love, and guidance have made this journey possible. Thank you for refusing to give up on me.

A special dedication to the family who loved and supported me when I started graduate school, but are now watching over me from heaven, Uncle Mike and Grandma Campbell. Your love and support live on in my heart. And a final dedication to Daisy, the world's most wonderful dog, who is no longer by my side but will forever be in my heart.

God gave us memory so that we may have a Daisy in December.

(Adapted from James M. Barrie) 


\section{Acknowledgements}

Graduate school tests one's ability to stand up after falling down. The following groups and individuals lent me a hand when I had to stand back up but did not know how.

\section{Family and Friends:}

I am blessed to have so many wonderful, supportive people in my life who, despite my occasional reluctance, have pushed me towards my goals. This amazing accomplishment is not only a reflection of my perseverance, but of theirs as well. I cherish being able to share it with each and every one of them.

Mom and dad, you both have supported me more than could be expressed in words. Whenever I have needed you, no matter the time, you answered and listened. You gave me advice when I needed it and a gentle nudge when I required it. There was a time in my life when I was not sure I would make it to college, let alone graduate school; thank you for never giving up hope and for always believing that I could do anything I put my mind to.

Mandi, Austin, and Abby, you both know how trying graduate school is. Thank you for always lending me your ear when I needed it and for sharing in all of my highs and lows. I am so lucky to have siblings that are also my friends. Kelsey, thank you for supporting me from back home and continuing to be my best friend in life. I am blessed to have had you as a best friend for the last twelve years; here's to twelve more. Violet, I could not have asked for a better West Virginia best friend during my time in graduate school. Thank you for being my family when I needed it and a friend the rest of the time.

The past and present member of the Bell Research Group at West Virginia University, thank you for your guidance and, most importantly, your friendship. Brittany Stevens (a.k.a. Mama B), you have gone above and beyond what a past group member needed to; thank you for continuing to provide friendship, support, and guidance after you graduated. Bill Feeney, I could not have asked for a better friend and colleague to be the other half of a 'dynamic duo'. Your support is unwavering, and I am lucky to have you as a lab mate. I would also like to thank the tireless efforts of my undergraduate research assistants over the last four years: James Maxella, Megan Messet, Victoria Zeger, Ben Alderson, and Sydney Brooks.

\section{Faculty and staff:}

Becky Secrist, the department is blessed to have you. You have impacted the life of every graduate student who has come through the department, but I would like to thank you especially for the impact you have had on me. Your smiling face has uplifted me, and your help was appreciated beyond words.

Last, but not least, I would like to thank every one of my committee members, Dr. Suzanne Bell, Dr. Stephen Valentine, Dr. Jonathan Boyd, Dr. Glen Jackson, and Dr. Patrick Callery, for reviewing my dissertation and shaping my scientific thinking. Dr. Bell, thank you for putting up with my antics and pushing me up and over the graduate school mountain. 


\section{Table of Contents}

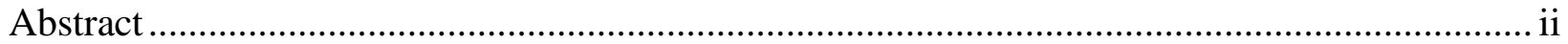

Acknowledgements ..................................................................................................

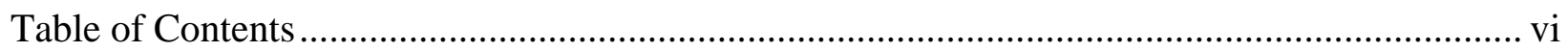

List of Figures ...................................................................................................... ix

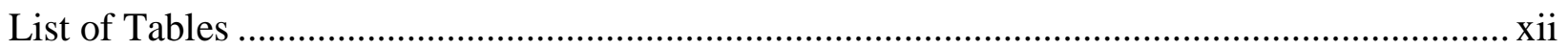

List of Abbreviations, Symbols, Nomenclature...................................................................

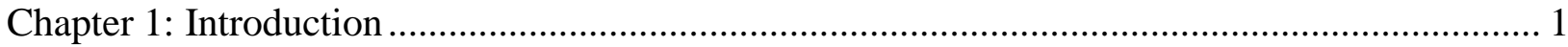

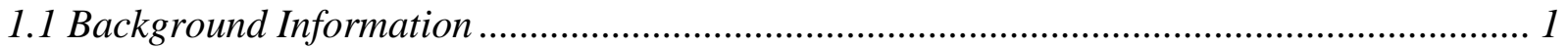

1.1.1 Definition of Novel Psychoactive Substances ........................................................... 1

1.1.2 Relevant Legislation .................................................................................................. 3

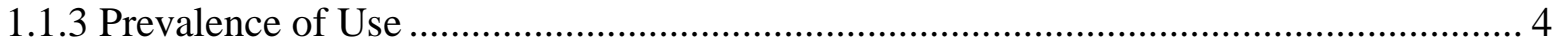

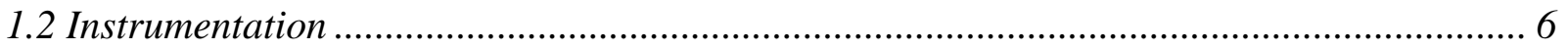

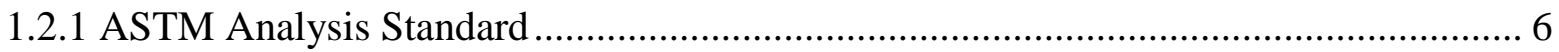

1.2.2 Gas Chromatography Mass Spectrometry ................................................................ 7

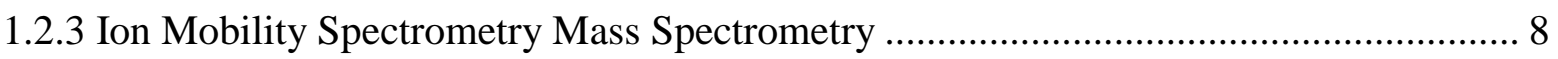

1.2.4 Hydrogen Deuterium Exchange ………………….................................................. 10

1.3 Purpose

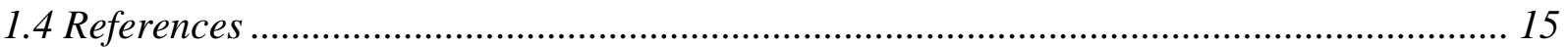

Chapter 2: Evaluation of the Reproducibility and Repeatability of GCMS Retention Indices and

Mass Spectra of Novel Psychoactive Substances. ........................................................................ 20

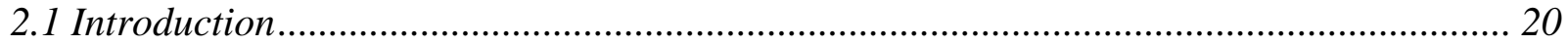

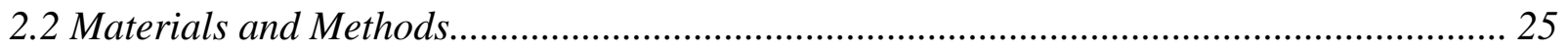

2.2.1 Chemical and Reagents ........................................................................................... 25

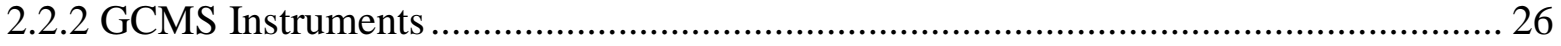

2.2.3 Experimental Design and Data Analysis ....................................................................... 28

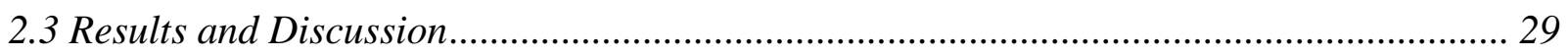

2.3.1 Retention Indices ................................................................................................... 30

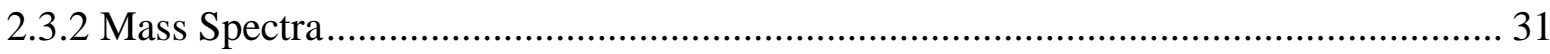




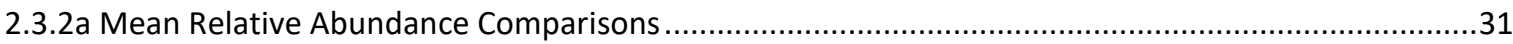

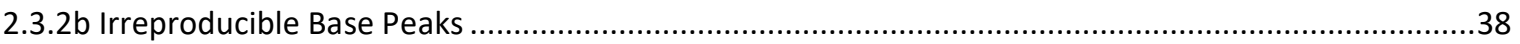

2.4 Conclusion and Next Steps......................................................................................... 40

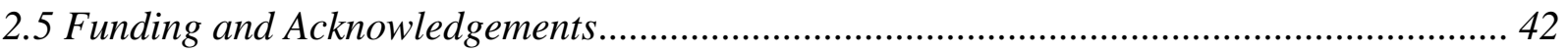

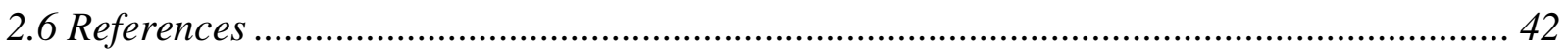

Chapter 3: The Effect of Mass Spectrometry Tuning Frequency and Criteria on Ion Relative

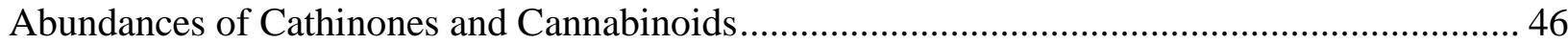

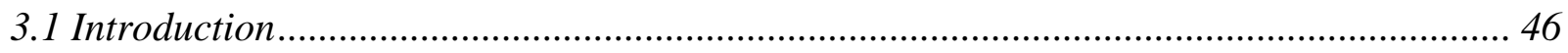

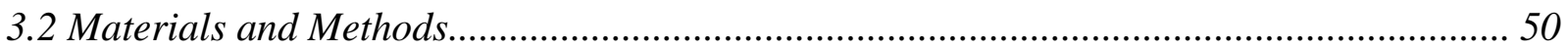

3.2.1 Chemicals and Reagents ........................................................................................... 50

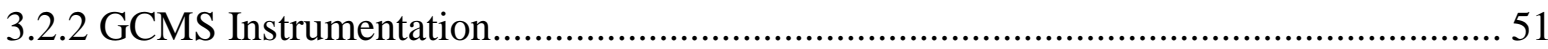

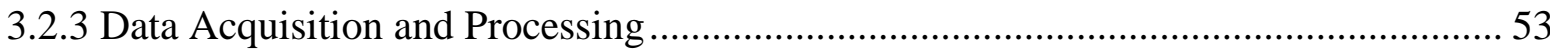

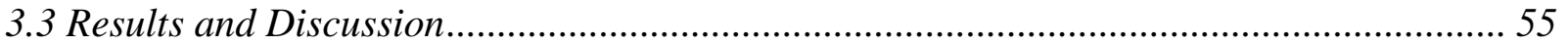

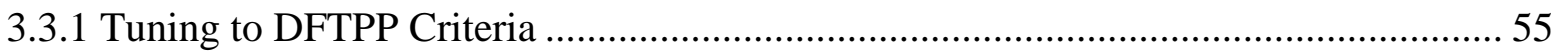

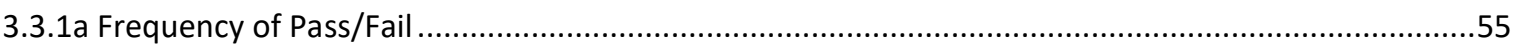

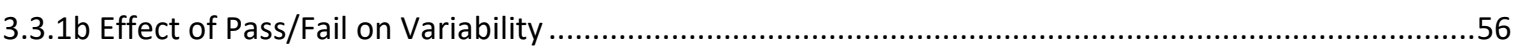

3.3.1c t-test of Means and f-test of Variance Tune Pass/Fail ...................................................................58

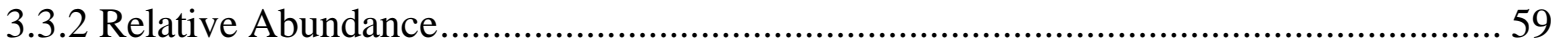

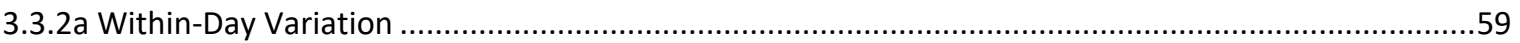

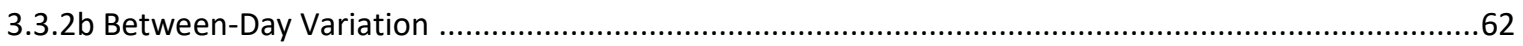

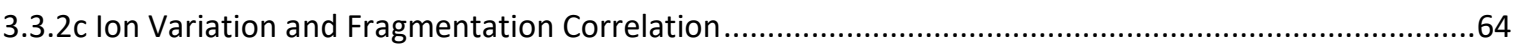

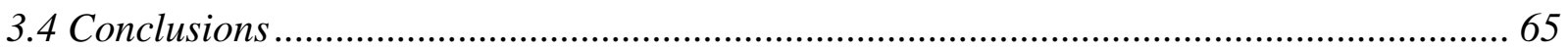

3.5 Funding and Acknowledgements.................................................................................... 66

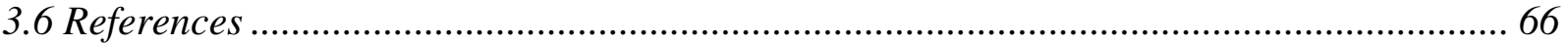

Chapter 4: Synthetic Small Molecule Characterization and Isomer Discrimination Using Gas-

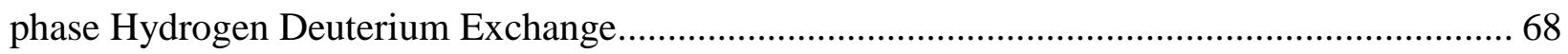

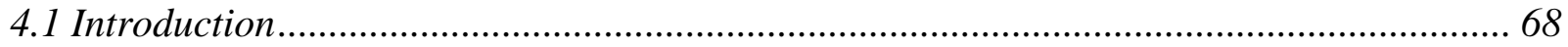

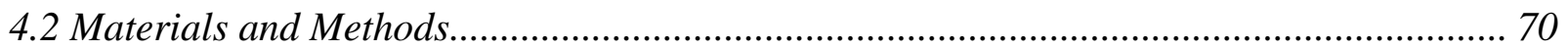

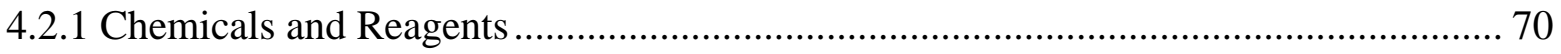

4.2.2 Experimental Design ......................................................................................... 71

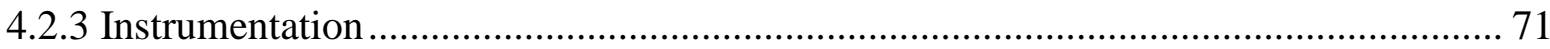


4.2.4 Collisional Cross Section Calculation ....................................................................... 73

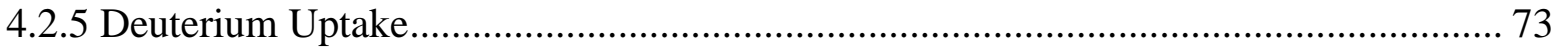

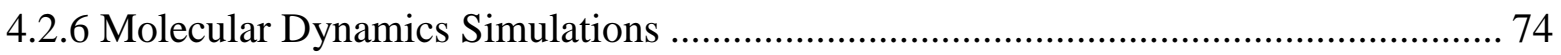

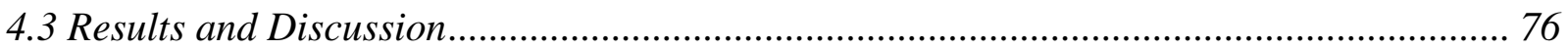

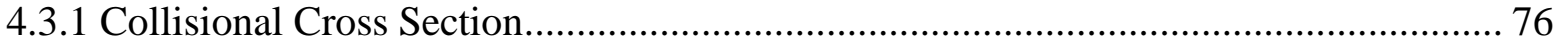

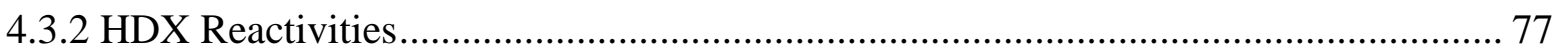

4.3.3 Discrimination of Disubstituted Aromatic Ring Isomers ........................................... 79

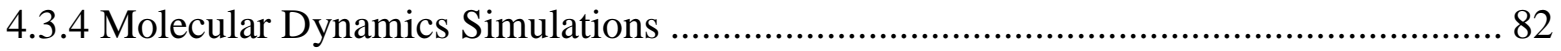

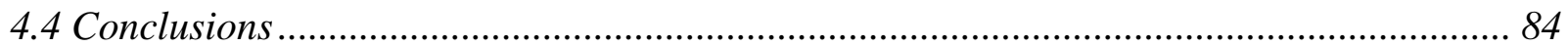

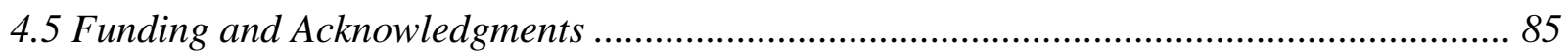

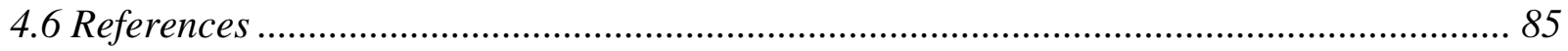

Chapter 5: Conclusions and Future Directions ………………………………………............... 93

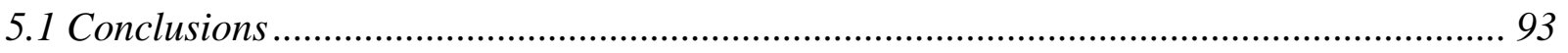

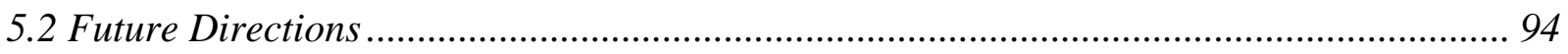

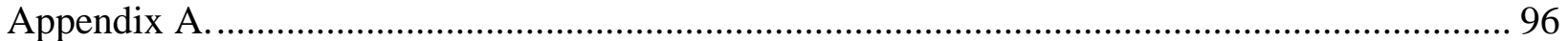

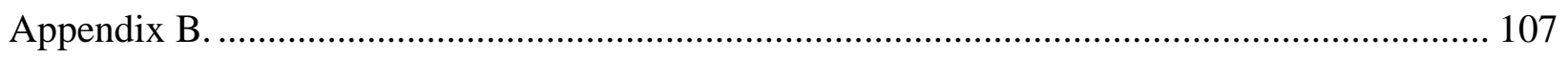

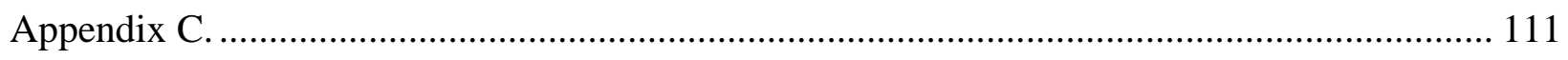




\section{List of Figures}

Figure 1.1. Schematic of GCMS instrumentation........................................................ 7

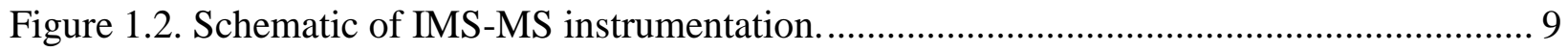

Figure 2.1. Flow chart of how a sample set was determined to be included or discarded........... 28

Figure 2.2. Means comparison of RIs for all NPSs. .......................................................... 31

Figure 2.3a. Example of feature-poor spectra (amphetamine). ........................................... 32

Figure 2.3b. Example of feature-rich spectra (JWH-018). ............................................... 32

Figure 2.4. Means comparison of \%RA of five most abundant ions for all NPSs. ................... 34

Figure 2.5a. Amphetamine box means comparison chart for $m / z$ 91 ..................................... 35

Figure 2.5b. Amphetamine box means comparison chart for $m / z$ 65 ................................... 36

Figure 2.5c. Amphetamine box means comparison chart for $m / z$ 42 ................................... 36

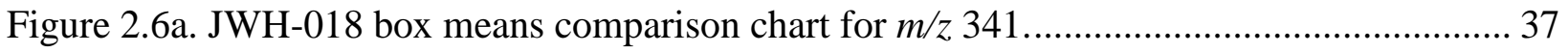

Figure 2.6b. JWH-018 box means comparison chart for $m / z$ 284 ........................................ 37

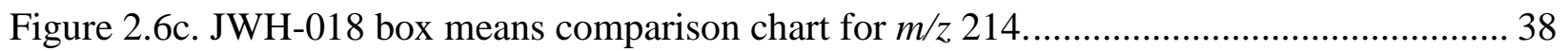

Figure 3.1. Collection and collation of data procedure ..................................................... 53

Figure 3.2. Comparison of spectra classified as feature-rich and feature-poor. ........................ 55

Figure 3.3a. Mean \% relative abundance per day for select ions from NEB separated by DFTPP

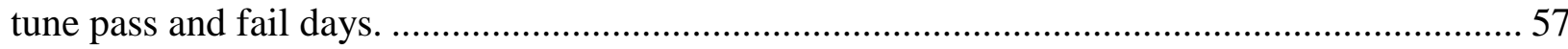

Figure 3.3b. Mean \% relative abundance per day for select ions from JWH-200 separated by

DFTPP tune pass and fail days.

Figure 3.3c. Mean \% relative abundance per day for select ions from JWH-149 separated by

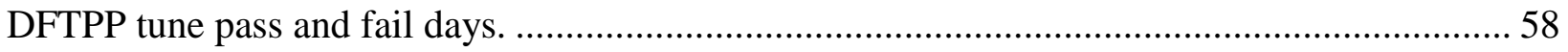

Figure 3.4. Comparison of performance as a function of tune pass/fail. ................................. 59

Figure 3.5. Relative standard deviation plotted as a function of the mean relative abundance from single day analysis sets $(n=10)$ from all NPSs and separated by NPS category and sub-class. ... 61 Figure 3.6a. Mean RA per day for select ions from NEB................................................ 63

Figure 3.6b. Mean RA per day for select ions from JWH-200............................................ 64

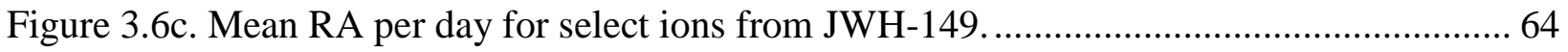

Scheme 4.1a. Structure of ortho-fluorophenyl piperazine highlighting the two potential deuterium exchange sites. 75 
Scheme 4.1b. Example of the atoms labelled for calculation of the dihedral angle. 75

Figure 4.1. Isotopic distributions for benzyl piperazine.

Figure 4.2a. Isotopic distributions for all fluorophenyl piperazine isomers when $\mathrm{D}_{2} \mathrm{O}$ is used as the reagent gas. 81

Figure 4.2b. Isotopic distributions for all fluorophenyl piperazine isomers when $\mathrm{ND}_{3}$ is used as the reagent gas.

Figure 4.3. Dihedral angle between the aromatic ring and diamine ring for all fluorophenyl piperazine isomers. 83

Figure A1a. \%RSD plotted as a function of mean \%RA for all three instruments.................... 96

Figure A1b. \%RSD plotted as a function of mean \%RA for instruments A and B................. 96 Figure A2. Three-dimensional plot comparing $m / z$, mean \%RA and \%RSD. Boxed data points are outliers which were investigated. 97

Figure A3. 3-dimensional wall projection. 97

Figure A4. 1-(3-chlorophenyl)piperazine mass spectra with box comparison charts of the top five ions. 102

Figure A5. 2,5-dimethoxy-4-iodoamphetamine mass spectra with box comparison charts of the top five ions 103

Figure A6. 25C-NBOMe mass spectra with box comparison charts of the top five ions. 103

Figure A7. UR-144 mass spectra with box comparison charts for top five most abundant ions.104 Figure A8. 5-MeO-AMT mass spectra with box comparison charts for top five most abundant ions. 104

Figure A9a. Chromatogram from Yellow mix. ............................................................... 105

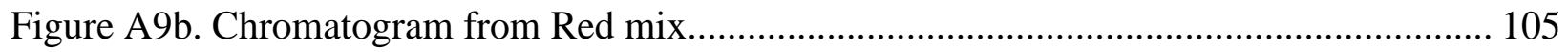

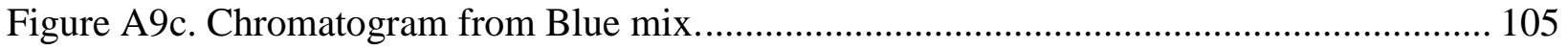

Figure B1. \%RSD plotted as a function of the mean RA from single day analysis sets from all

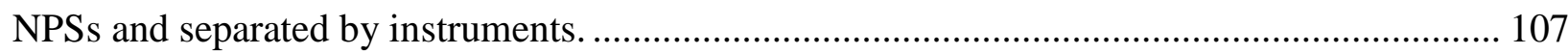

Figure B2a. All recorded RAs for select ions from NEB. ................................................ 108

Figure B2b. All recorded RAs for select ions from JWH-200. ........................................ 108

Figure B2c. All recorded RAs for select ions from JWH-149......................................... 108

Figure B3a. All recorded RAs for select ions from MeMABP............................................ 109

Figure B3b. All recorded RAs for select ions from JWH-116. ........................................ 109 
Figure B3c. All recorded RAs for select ions from BB-22 ............................................ 109

Figure B4. Sample chromatogram for NPS solutions.................................................. 110

Figure C1. Schematic of the home-build drift tube used.................................................... 111

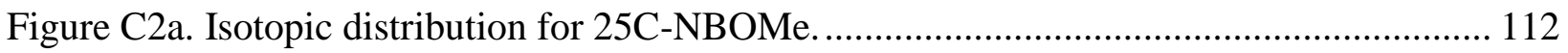

Figure C2b. Isotopic distribution for 4-fluoroamphetamine............................................ 113

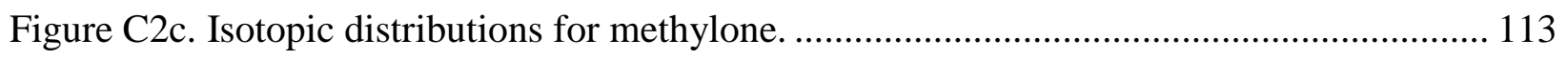

Figure C2d. Isotopic distributions for AH-7921 ............................................................ 114

Figure C2e. Isotopic distributions for 5-MeO-AMT. ...................................................... 114

Figure C3. Isotopic distributions for all fluorophenyl piperazine isomers in buffer gas........... 115

Figure C4. Comparison of deuterium uptake for the fluorophenyl piperazine isomers. ........... 116

Figure C5a. Dihedral angle between the aromatic ring and the diamine ring for ortho-

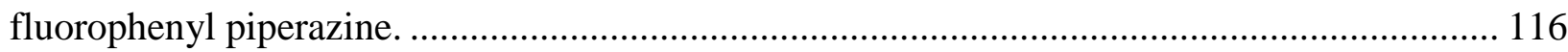

Figure C5b. Dihedral angle between the aromatic ring and the diamine ring for meta-

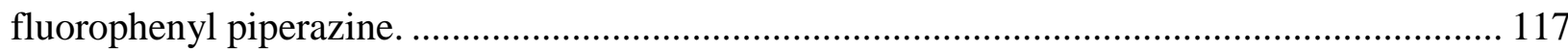

Figure C5a. Dihedral angle between the aromatic ring and the diamine ring for para-fluorophenyl

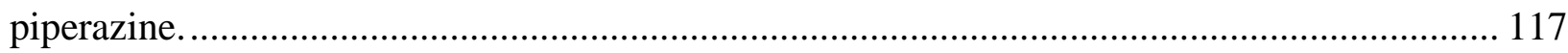




\section{List of Tables}

Table 2.1. NPSs contained in each of the three mixes....................................................... 26

Table 2.2. Model number and method settings for GCMS systems. ..................................... 27

Table 2.3. Complete analysis in order which were performed. ............................................ 28

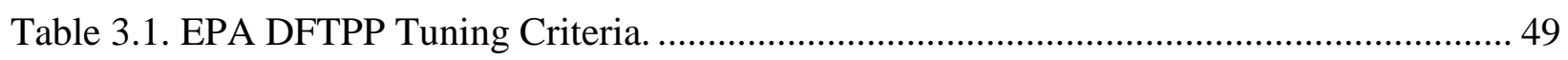

Table 3.2. Composition of Check Mix and NPS drug mixes............................................. 50

Table 3.3. GC-MS systems details, method/instrument settings, and instrument/analysis software.

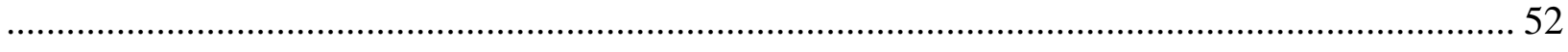

Table 4.1.CCS values and deuterium uptake for all compounds......................................... 77

Table A1. Descriptive statistics for RIs for all NPSs..................................................... 98

Table A2. Descriptive statistics for five most abundant ions for select NPSs......................... 99

Table C1. Experimental parameters used for CCS calculations. ........................................... 111

Table C2. Theoretical CCS mean and 95\% confidence interval range and dipole moments for the

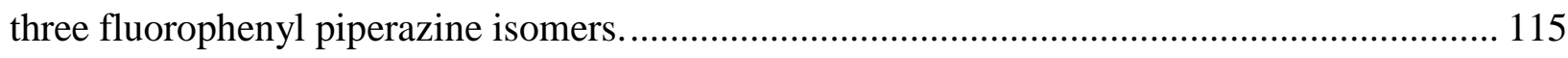




\section{List of Abbreviations, Symbols, Nomenclature}

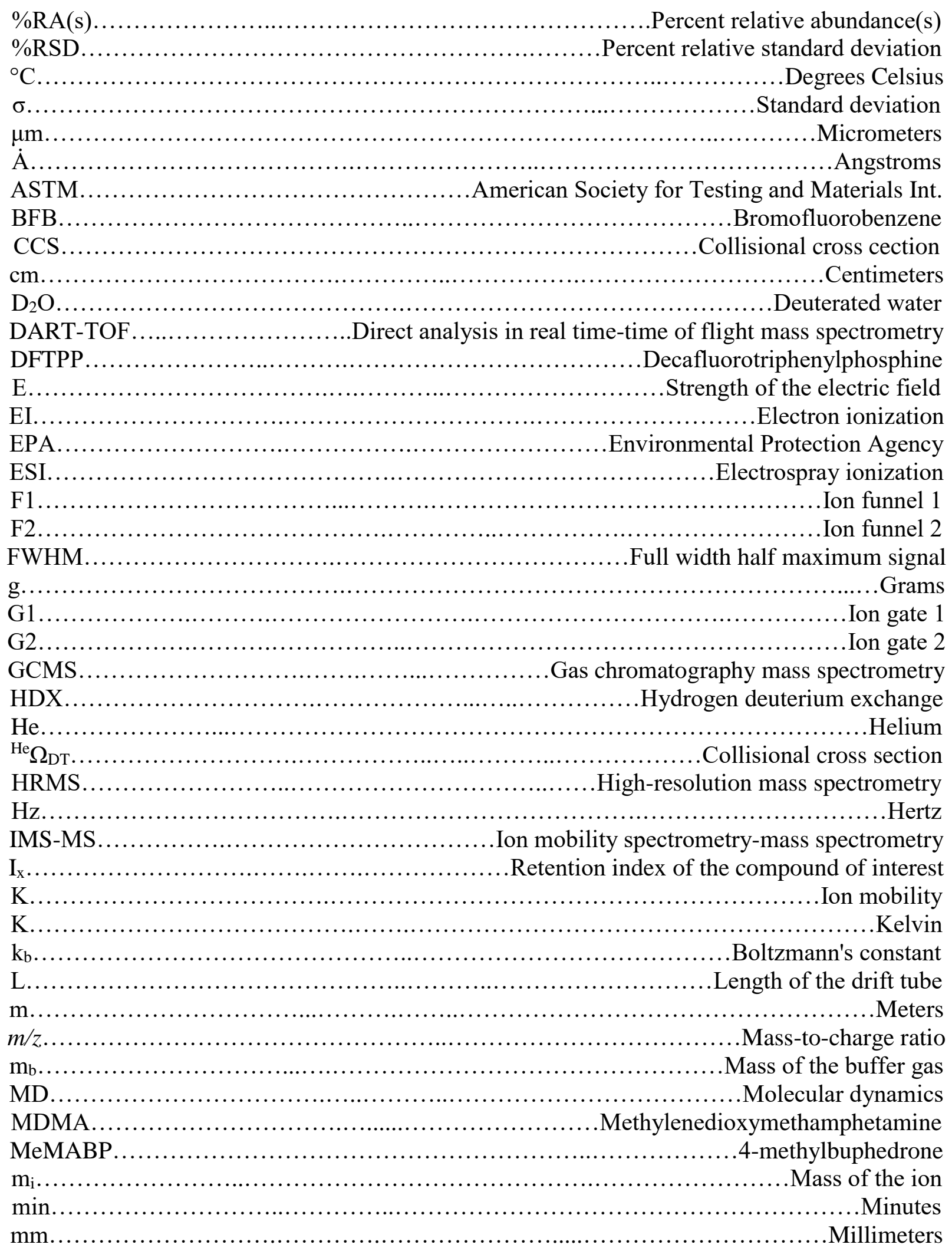


mol Moles

$\mathrm{ms}$ Milliseconds

$\mathrm{MS} / \mathrm{MS}$ Tandem mass spectrometry

n. N........ Number of carbon atoms in alkane eluting prior to compound of interest $\mathrm{ND}_{3}$ Density of the buffer gas at STP

NEB Deuterated ammonia

NIST N-ethylbuphedrone

$\mathrm{nL}$ National Institute for Standards and Technology

$\operatorname{NPS}(\mathrm{s})$ Nanoliters P. Novel psychoactive substance(s) PFTBA .Pressure of the buffer gas ppm Perfluorotributylamine RI(s) Parts per million $\mathrm{RI}(\mathrm{s})$ Retention index (indices) STP Standard temperature and pressure SWGDRUG. Scientific Working Group on the Analysis of Seized Drugs $\mathrm{T}$ Temperature

$t_{D}$

$t_{n}$ Retention time of alkane eluting prior to compound of interest

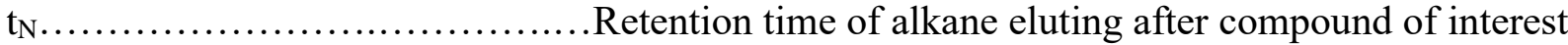
$\mathrm{t}_{\mathrm{x}}$ Retention time of compound of interest

UNODC. United Nations Office on Drugs and Crime US United States

$\mathrm{V}$ Voltage ze. Charge of the

ion

\section{IUPAC Naming}

2,5-dimethoxy-4-iodoamphetamine.............1-(4-iodo-2,5-dimethoxyphenyl)propan-2-amine 25C-NBOMe..............2-(4-chloro-2,5-dimethoxyphenyl)-N-(2-methoxybenzyl)ethanamine 25I-NBOMe.................2-(4-iodo-2,5-dimethoxyphenyl)-N-(2-methoxybenzyl)ethanamine 4-fluoroamphetamine.................................... (4-fluorophenyl)propan-2-amine 5-MeO-AMT .................................. 5-methoxy-1H-indol-3-yl)propan-2-amine AH-7921...................3,4-dichloro-N-\{[1-dimethylamino)cyclohexyl]methyl $\}$ benzamide Amphetamine.......................................... RS $)$-1-phenylpropan-2-amine BB-22. .quinolin-8-yl 1-(cyclohexylmethyl)-1H-indole-3-carboxylate Benzyl piperazine.........................................1-(phenylmethyl)-piperazine Butylone.............................. (1,3-Benzodioxol-5-yl)-2-(methylamino)butan-1-one JWH-018 naphthalen-1-yl(1-pentyl-1H-indol-3-yl)methanone

JWH-116. .(2-ethyl-1-pentyl-1H-indol-3-yl)-1-naphthalenylmethanone JWH-149 (4-methyl-1-naphthalenyl)(2-methyl-1-pentyl-1 $H$-indol-3-yl)methanone JWH-200............................... 1-[2-(4-morpholinyl)ethyl]-3-(1-naphthoyl)indole 4-Methylbuphedrone.........................2-(methylamino)-1-(4-methylphenyl)-1-butanone Mephedrone............................2-(methylamino)-1-(4-methylphenyl)propan-1-one Meta-fluorophenyl piperazine..............................1-(3-fluorophenyl)piperazine 
Methylone. ...

1-(1,3-benzodioxol-5-yl)-2-(methylamino)propan-1-one N,N-Dimethyltryptamine ..................................... N-Ethylbuphedrone. 2-(Ethylamino)-1-phenylbutan-1-one Ortho-fluorophenyl piperazine..............................1-(2-fluorophenyl)piperazine

Para-fluorophenyl piperazine. .1-(4-fluorophenyl)piperazine UR-144. .(1-pentyl-1H-indol-3-yl)(2,2,3,3-tetramethylcyclopropyl)methanone 


\section{Chapter 1: Introduction}

\subsection{Background Information}

\subsubsection{Definition of Novel Psychoactive Substances}

Novel psychoactive substances (NPSs) are a family of synthetically derived compounds designed to mimic the effects of other illicit drugs. By United Nations Office on Drugs and Crime (UNODC) definition, these are psychoactive compounds, either pure or in prepared mixtures, that pose a similar public health concern as controlled substances but themselves are not under current legislation. The term 'novel' directly refers to availability for abuse and misuse but not necessarily to first synthesis. ${ }^{1-2}$ Herein, NPSs will refer to both substances which currently fit this definition as well as substances which previously fit the definition.

Numerous terms have previously been used to describe NPSs including designer drugs and legal highs. Although all three terms are interrelated there are subtle differences in the definitions established by international agencies. Designer drugs are substances specifically manufactured to escape existing drug control measures on national and/or international levels. These substances are structural derivatives of a parent compound and mimic the pharmacological behavior. On the other hand, legal highs refer to psychoactive substances escaping drug control measures in the country which they are sold. Legal high substances are designed to mimic behavior of a specific compound with or without structural similarity. The term NPSs, defined above, was established to streamline policy making and includes both designer drugs and legal highs. ${ }^{1}$

NPSs can be analogues or mimetics. An analogue is a structural derivative of a parent compound usually containing small side chain substitutions. Importantly, analogues may or may not have similar pharmacological properties as the parent compound. On the other hand, 
mimetics are structurally different but mimic the pharmacological activity of a particular substance by acting on the same the same receptor. ${ }^{1}$ NPSs are also classified into categories, also known as groups, and families within each category. Categories refer to a collection of mimetic compounds related to a specific regulated substance; each category can contain multiple families. NPS families are analogues with side chain substitutions or structural rearrangements that have varying effects on pharmacological activity. Because the backbone or base structure of families are nearly identical, structural isomers and isobaric compounds have been identified, and, as described below, this can make identification challenging.

There are seven NPS categories defined by the UNODC; the two largest categories are synthetic cannabinoids and cathinones ${ }^{1-8}$, these are described in more detail below. Other categories include phenethylamines, piperazines, ketamine, plant-based substances, and miscellaneous. ${ }^{2}$ Alternative groupings (including opioid-like drugs, tryptamines, and aminoindanes) have been proposed in literature to further classify the wide-range of compounds which fall under the miscellaneous umbrella term. ${ }^{1,4-5,9-10}$

Synthetic cannabinoids or cannabimimetics are the most prolific category containing approximately 14 different families. ${ }^{1,-7}$ This category of substances interacts with $\mathrm{CB}_{1}$ and $\mathrm{CB}_{2}$ cannabinoid receptors and mimic the effects of $\Delta^{9}$-tetrahydrocannbinol, the primary active compound in cannabis. ${ }^{6}$ Synthetic cathinones, or bath salts, are $\beta$-ketoamphetamines which are structurally similar and mimic the effects of amphetamines, methylenedioxymethamphetamine (MDMA), and cocaine. These NPSs are derivatives of cathinone, the active compound extracted from the Khat plant (Catha edulis). ${ }^{4,8,11}$ Two distinct groups exist within this category, semisynthetic and synthetic, which are cathinone derivatives and MDMA-like lab created compounds, respectively. ${ }^{8}$ 


\subsubsection{Relevant Legislation}

NPSs fall outside of the international drug control system and are therefore not included in neither the 1961 Convention nor the 1971 Convention. ${ }^{3}$ Despite the Expert Committee on Drug Dependence of the World Health Organization reviewing several NPSs, these compounds in large have not been adopted into the current international scheduling agreements. ${ }^{3}$ As a result, regions and individual nations have adopted legislation to address the growing epidemic. Of particular relevance to this work is the United States (US) which adopted the Controlled Substances Act of 1970. ${ }^{12}$ This act uses various factors in order to categorize substances into five groups, termed schedules. ${ }^{12}$ According to subsection (h) of the act, substances can be temporarily scheduled for 2 years as a means of avoiding imminent public health safety. ${ }^{12}$ However, the Controlled Substances Act defines substances by the unique and specific molecular structure of the compound.

Because the US drug regulations are largely limited by chemical structure of the active substance or substances, the small side chain substitutions of NPS families allow newly available analogues to evade drug regulations at the time they are released to the drug market. ${ }^{1,4-5,7}$ In an attempt to prevent this creative circumvention of the Controlled Substances Act, the US enacted the Anti-drug Abuse Act of $1986 .^{13}$ This act effectively extended drug legislation of specific drug schedules to analogues so long as the compound is marketed for human consumption. ${ }^{13}$ However, NPSs are marketed worldwide as research chemicals, air fresheners, plant food, and bath salts. Disclaimers are also often present on packaging labels, such as 'not for human consumption' and 'for research purposes only'. It is these labels and disclaimers that aide NPSs to temporarily circumvent not only the updated US drug legislation, but the legislation of countries worldwide..$^{4-5}$ 


\subsubsection{Prevalence of Use}

Two studies, described in detail below, examined whether the national scheduling of NPSs was effective at deterring the overall interest in these illicit drugs and whether legislation was effective in preventing the sale of the scheduled substances.

In 2015 Ledberg ${ }^{14}$ analyzed Swedish online forum chatter from over 3800 websites and 3,700 individual users. These websites were separated into eight different threads, one for each specific NPS studied. Using a time-series model, the activity levels on each thread were studied for correlation between intensity of activity, defined as the posts per day, and the date of substance regulation, determined to be the 180 days before and the 180 days after scheduling occurred. For seven of the eight NPSs, there was a strong statistical association between the legal status and activity relating to the substance; as the legal status changed there was a statistically significant reduction in online activity mentioning the substance. The last substance showed a statistically significant reduction in activity when the time-series window was doubled. Also investigated was whether the reduction in activity was due to members losing general interest in drugs or whether the members were turning to other drug forums to continue their online activity. The activity of a random subset of users was followed for the 180-day time-series window from five of the study's threads. For three of the five investigated NPS threads, the selected member's online activity was diverted to another thread; this indicated that while the interest in the particular NPS decreased after the legal status change, the general interest in NPSs did not. ${ }^{14}$ Although this study was conducted specifically focusing on Swedish online forums, it shows that a likely scenario is the diversion of interest from a newly scheduled substance to a replacement. Furthermore, the author indicates that although this data shows correlation it does 
not speak to causation; meaning that the reduction in online discussion activity could be the result of the product being removed from circulation in head shops or online distributors. ${ }^{14}$

Belackova et. al ${ }^{15}$ studied whether the online accessibility and availability of select NPSs changed after controlling laws were enacted in five European countries. ${ }^{15}$ The authors defined accessibility as the number of unique e-shops identified as selling the specific substance per country; whereas availability was defined as whether or not the e-shop was stocked and shipping the substance to the each of the countries at two specific time points. The first time point was chosen to be shortly before the risk assessment of the NPSs of interest was released by the European Monitoring Centre for Drugs and Drug Addiction in 2014, while the second was in late 2015 shortly after the control measures were adopted by the European Union states for the compounds of interest. From the first to second time point the number of e-shops selling at least one of the four NPSs decreased by half, 33 e-shops after control compared to 66 before the risk assessment. Only nine shops were identified in both sample sets. This means that 57 e-shops ceased to exist, no longer sold any products, or did not offer the NPSs of interest and 24 new eshops selling the controlled substances appeared. Despite the appearance of new e-shops, the overall accessibility of the compounds of interest decreased, but the changes in the availability were compound and country specific. For instance, the authors noted an increase in the availability of AH-7921 in France and Poland after the control measures were adopted compared to before. With the exception of 25I-NBOMe in the Netherlands, all compounds available in 2014 were still available in 2015; the authors suggest this is evidence to the ineffectiveness of country specific legislation. ${ }^{15}$

Together these studies indicate that while the packaging loophole is being exploited, the enacting of legislation to control individual compounds may not be effective in managing, or 
even slowing, the NPS epidemic. In a seemingly never-ending cycle, once a newly characterized NPS is scheduled, an alternative, unscheduled analogue can act as a replacement but the original NPS may still be available on the market. This in sum creates a unique problem for forensic scientists- whether the substance being tested is a new, uncharacterized NPS; whether the substance has been characterized but is not controlled by drug regulations; or whether the NPS is unidentified, uncharacterized, and new to the drug market. Forensic scientists are now tasked with distinguishing between structural isomers and isobars in order to determine the legal status of the compound; therefore, analytical detection and characterization methods as well as the uncertainty behind each technique used are more important now than historically in forensic drug chemistry.

\subsection{Instrumentation}

\subsubsection{ASTM Analysis Standard}

A UNODC survey from 60 countries and territories showed the most often used method for identification of NPSs was chemical analysis techniques, including gas chromatography-mass spectrometry (GCMS) and ion mobility spectrometry (IMS); the second was the use of reference standards. $^{3}$ The American Society for Testing and Materials Int. (ASTM) defines the minimum criteria for qualitative identification of seized drugs in ASTM E2329-17. ${ }^{16}$ In this standard practice, the analytical techniques are categorized into three groups (A, B, and C) based on maximum potential to discriminate between drug compounds, where category A has the highest discrimination power. By definition, when a category A technique is used a second and different technique, from A, B, or C, must also be employed. If a category A technique is not used, then

three different techniques at least two from category B must be used. Hyphenated techniques, i.e. GCMS and IMS-MS, are considered two separate techniques, where GC and IMS are category B 
and MS is a category A; therefore, these techniques meet the ASTM discrimination criteria. This definition is in agreement with the recommendations set forth by the Scientific Working Group on the Analysis of Seized Drugs (SWGDRUG). ${ }^{17}$ Additionally, SWGDRUG recommendations acknowledge that the discrimination power of any technique may be lower than the classification if the sample, analyte, or mode of operation hinders the data's discrimination. One example provided by SWGDRUG would be a MS technique that only provides molecular weight information. ${ }^{17}$

\subsubsection{Gas Chromatography Mass Spectrometry}

A basic schematic of GCMS instrumentation is shown in Figure 1.1. A liquid sample, consisting of the analyte(s) of interest dissolved in an organic solvent, is injected into the injection port and is near instantaneously vaporized. An inert gaseous mobile phase is used to move the analytes through a capillary column located within an oven. Separation is the result of the analytes partitioning between gaseous mobile phase and stationary phase in the column. Temperature programing is used to improve separation by the slow and controlled oven temperature increase. Compounds with similar boiling points will have different rates of vaporization/condensation creating a larger separation between compounds. ${ }^{18}$ The retention time refers to the time at which compounds elute from the column into the mass spectrometer.

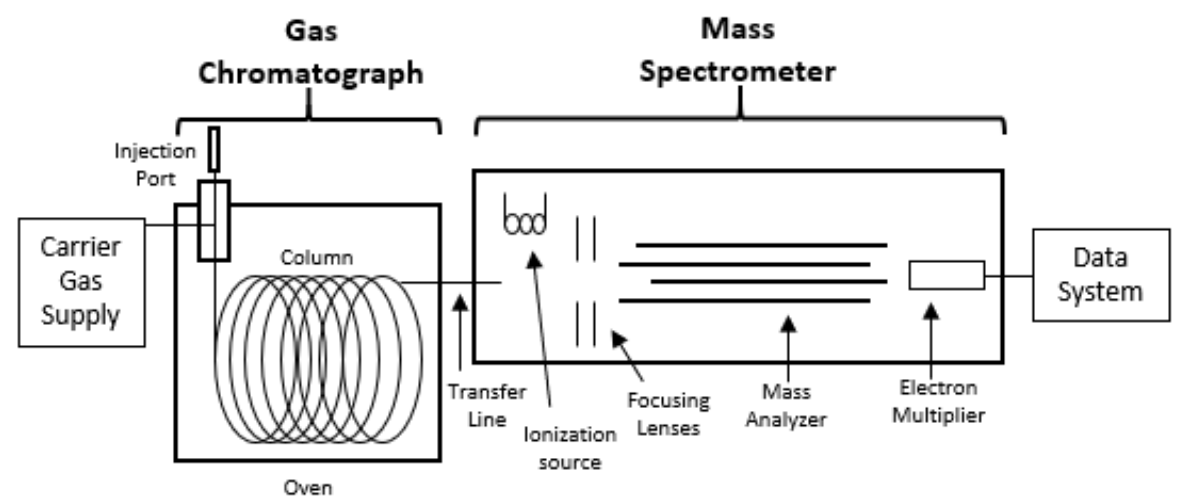

Figure 1.1. Schematic of GCMS instrumentation. 
The most common type of mass spectrometer coupled to GC is a quadrupole mass filter capable of both electron impact (EI) and chemical ionization. In electron impact ionization, an energetic electron collides with a neutral molecule transferring kinetic energy. ${ }^{19}$ An effective collision leaves the once neutral molecule as a positively charged ion; the ion will fragment when the internal energy of the cation exceeds the dissociation energy level. A quadrupole mass filter separates the fragment ions by creating an oscillating electric field between four rods. Ions of the correct mass-to-charge $(\mathrm{m} / \mathrm{z})$ value maintain a stable trajectory and enter the detector, while all other $\mathrm{m} / \mathrm{z}$ values experience an unstable trajectory and are ejected from the mass filter. A user specified range of $\mathrm{m} / \mathrm{z}$ values is scanned repeatedly, producing a mass spectrum containing all the fragment ions and, when present, the parent ion. ${ }^{19}$ The signal intensity of a mass spectra is displayed in terms of percent relative abundance (\%RA), where the intensity of all ions is scaled to the most intense ion signal detected.

GCMS analyses produce two types of data that can be used to identify unknown analytes of interest: a chromatogram showing the retention time and a mass spectrum. Retention times cannot be compared across all methods because the time of elution is temperature dependent. A value known as retention index (RI) was developed in order to compare data between methods. The mass spectra of analytes are either compared manually against mass spectra from certified reference material, also known as a standard, or a mass spectral library search is performed.

\subsubsection{Ion Mobility Spectrometry Mass Spectrometry}

A basic schematic of a drift tube IMS-MS is shown in Figure 1.2. Although many ionization sources can be used, of relevance to this work is electrospray ionization (ESI). The liquid sample solution (containing the analytes, organic solvent, and dilute organic acid) is 
aerosolized through a spray capillary carrying a potential. The aerosol consisting of $\mu \mathrm{m}$ sized charged droplets expands into a heated desolvation region and undergoes vaporization. Vaporization is the result of repetitive shrinking and droplet disintegration through either the charge-residue model or the ion evaporation model. Although each model has sufficient supporting evidence, it is widely accepted that small molecule ion formation is best described by the ion evaporation model, where the analyte of interest evaporates directly from the highly charged droplet surface. ${ }^{19}$

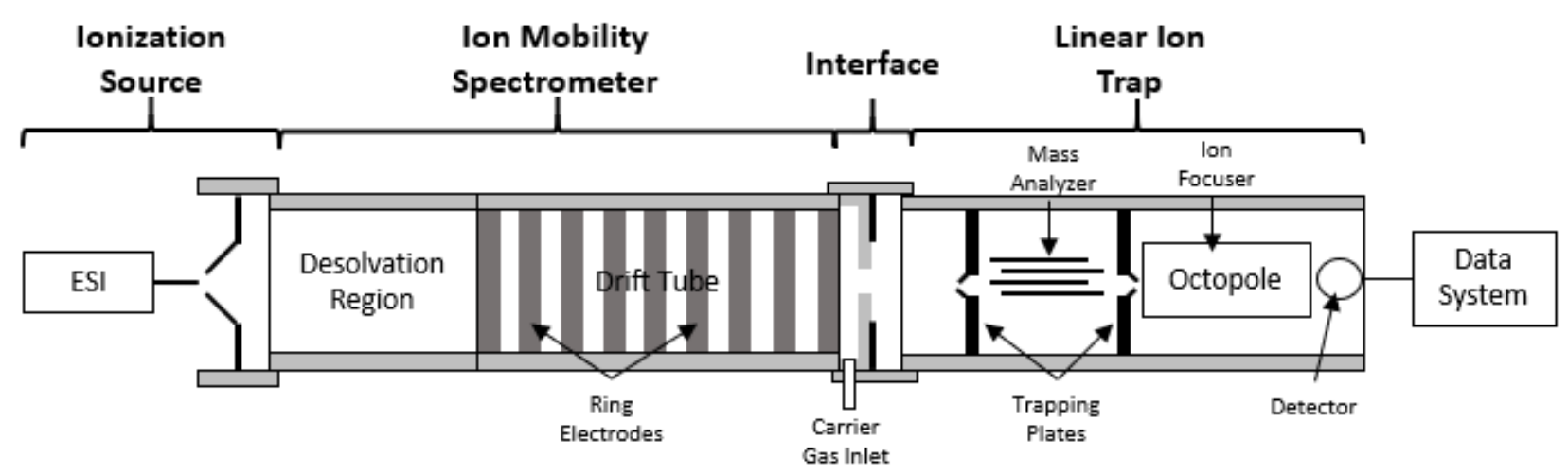

Figure 1.2. Schematic of IMS-MS instrumentation.

Ions are then periodically pulsed into the drift tube. Ring electrodes accelerate ions through the drift region while a neutral carrier gas flows opposite to the analytes. Larger, elongated molecules are slowed by more frequent collisions with the carrier gas, while smaller, compact molecules experience fewer collisions. Thus, separation is based on a molecule's sizeto-charge ratio. Although a multitude of mass spectrometers can be coupled to IMS, the linear ion trap is relevant to this work. When the front and back trapping plates have a low and high potential, respectively, the ions are allowed to enter the quadrupole; the front potential is quickly increased thereby trapping the ions between the two plates. Similar to the description above, the quadrupole filters ions for specific $\mathrm{m} / \mathrm{z}$ values. The potential of the back-trapping plate is lowered and ions of the selected $\mathrm{m} / \mathrm{z}$ values exit into the octupole and are detected. 
IMS-MS analyses produce two types of data that can be used to identify unknown analytes of interest. The drift time, or the time it takes for an analyte to traverse the drift tube, can be used to calculate the ion mobility (K). An ion mobility (Equation 1.1) is the proportionality factor between the ion's velocity and the electric field strength; however, this value is dependent on experimental conditions and cannot be compared between instruments. $K=\frac{V_{D}}{E}$

eq. 1.1 where $\mathrm{K}$ is the ion mobility, $\mathrm{E}$ is the electric field strength, and $\mathrm{V}_{\mathrm{D}}$ is the average velocity of a drifting ion. Using the Mason-Schamp equation (Equation 1.2) the collisional cross section (CCS), or the mean of the collision area between the analyte of interest among all orientations and the neutral drift gas, can be found. It is important to note that K and CCS cannot be compared between different drift gases.

$\Omega=\frac{z e}{16 N} \sqrt{\frac{18 \pi}{k_{B} T}} \sqrt{\frac{1}{m_{i}}+\frac{1}{m_{B}}} \frac{t_{D} T_{a} E \times 760}{L P \times 273.2}$

where $\Omega$ is the CCS, ze is the ion charge, $N$ is the number density of the buffer gas, $k_{B}$ is Boltzmann's constant, $T_{a}$ is the absolute temperature, $m_{B}$ is the reduced mass of the buffer gas, $M$ is the reduced mass of the ions of interest, $L$ is the length of the drift tube, $T$ is the temperature of the drift gas, $P$ is the pressure of the drift gas, $E$ is the electric field, and $t_{D}$ is the drift time of the analyte of interest. The second type of data which can be used for identification is mass spectra, similar to those described earlier. The mass spectra produced by a linear ion trap generally contain the molecular ion peak, frequently not present in EI spectra, and can contain fewer fragmentation peaks due to the different ionization techniques.

\subsubsection{Hydrogen Deuterium Exchange}


Hydrogen deuterium exchange (HDX) describes reactions in which a labile hydrogen(s) on an analyte are replaced with deuteron(s) resulting in neutron mass gain. ${ }^{20}$ Although it is possible to exchange carbon bonded hydrogens, labile hydrogens are most often bonded to heteroatoms as they are generally more acidic and therefore more easily replaced. ${ }^{20} \mathrm{HDX}$ has been employed to magnify the structural information obtained during IMS analyses. It is been used both as a sample preparation technique, involving liquid phase reactions, and as an on-line technique where gas phase reactions occur within the instrument or during ionization. ${ }^{20-21}$ Liquid phase HDX has historically been used in drug discovery applications, namely for determining protein structural changes ${ }^{22-23}$; however, the use of this technique in complex mixture analysis is limited. Gas phase HDX, on the other hand, has recently been used to distinguish biomolecule isomers and conformation populations in complex mixture analyses. ${ }^{21,24-26}$ Of particular interest is work by Uppal et. al ${ }^{24}$ in which IMS-MS alone was unable to resolve several of the mono- and oligo- saccharides studied. ${ }^{24}$ The gas phase HDX rate was reported to be sufficiently distinct and capable of distinguishing glycan ions and linkage isomers. ${ }^{24}$ Additionally, fragment ions, regardless of the starting carbohydrate structure, could be distinguished and identified by the rate of deuteron uptake. Because NPSs are small compared to many proteins and peptides studied via HDX and IMS, Uppal et. al's ${ }^{24}$ work highlights that this technique is capable of distinguishing small biomolecules with minor differences. This, in turn, suggests that HDX has the potential to distinguish small synthetic molecules such as NPSs.

Gas phase HDX reaction mechanisms of various deuterated reagents were studied and proposed in the 1990's by Beauchamp et. al. ${ }^{27}$ Relevant to the work presented here, are the suggested reaction mechanisms for deuterated water and ammonia, $\mathrm{D}_{2} \mathrm{O}$ and $\mathrm{ND}_{3}$. Because the exchange mechanisms are different, the structural information which can be elucidated by 
analysis in the presence of each reagent is also different. It is therefore important to analyze each analyte with each reagent gas.

It was suggested that $\mathrm{HDX}$ involving $\mathrm{D}_{2} \mathrm{O}$ occurs via the 'relay mechanism'. Here, the hydrogen from the protonation site is transferred to the deuterated reagent molecule as the deuteron is shuttled to a less basic site on the analyte. Importantly, a charged shuttle molecule or additional chargeable site on the analyte must be in close proximity to the protonation site in order to balance the charges during the deuteron transfer. This proposed mechanism reflects the proton affinity of $\mathrm{D}_{2} \mathrm{O}$, which is too low to overcome the endothermicity of the exchange, and requires a long-lived intermediate reaction complex, involving the analyte, shuttle molecule, and deuterated water, to be formed. It was also noted that, due to the low proton affinity and longlived reaction complex, exchanges occurring with $\mathrm{D}_{2} \mathrm{O}$ are likely more discriminate, wherein not all labile hydrogens will undergo exchange.

Exchanges involving $\mathrm{ND}_{3}$ were proposed to occur via the 'onium ion mechanism'. This mechanism requires the simultaneous solvation of the onium ion as the deuteron is exchanged with a proton on the basic exchange site. Exchanges via the onium mechanism can only occur if the energy recovered from solvation is greater than the energy lost by the exchange. Accounting for this, the proposed exchange is considered nominally endothermic and comparatively faster by the authors. It was, therefore, suggested to be less discriminate and all labile hydrogens are exchanged on the analyte.

\subsection{Purpose}

Non-target analysis is a term commonly used in metabolomics and proteomics, where the identity of the analyte is a true unknown. Commonly, drug chemists analyze standards, or certified reference material, concurrently with case samples allowing for the manual comparison 
of retention time and mass spectral features. Due to the never-ending cycle of new NPSs entering the illicit drug market, which results in standards not being available, non-target analyses are becoming increasingly common in forensic drug chemistry. Furthermore, drug chemists are now forced to determine whether the NPS is a new, uncharacterized analogue; a known, characterized, but unscheduled analogue; or a known, characterized, and scheduled analogue.

Molecular structures fragment in predictable ways, namely, at the weakest bonds within a molecule. Mass spectrometry text books report that EI "mass spectra are excellently reproducible when measured under standard conditions... This is not only the case for repeated measurements on the same instrument, but also between different types and brands of mass spectrometers"19. Because the EI fragmentation patterns are shown to be reproducible and the \%RAs are presumed to be, a common strategy for non-target identifications is using mass spectral library searches. In a library search, all $\mathrm{m} / \mathrm{z}$ values and corresponding \% RAs within an unknown spectrum are compared to known standard spectra within the library and a mathematical algorithm returns a match quality score. Different libraries may contain multiple search algorithms. For example, the National Institute for Science and technology (NIST) database contains two search algorithms, Identity Search and Similarity Search. The former algorithm searches for exact matches while the latter is designed to identify similar compounds; the optimization and testing of these mathematical algorithms is detailed by Stein et. al. ${ }^{28}$ Over the years, mass spectral libraries have been evaluated for quality of spectra ${ }^{29}$ and match correctness ${ }^{30}$, while inter-library comparisons have worked to identify potential errors in reference spectra ${ }^{31}$. The most recent search algorithm, termed the Hybrid Similarity Search, developed by NIST and for the NIST library allows for neutral-loss and fragment ion matching and is applicable to illicit compounds. ${ }^{32}$ This search can classify and identify compounds not already in the database by predicting shifts due to 
substituent changes; however, successful prediction requires the molecular mass to be known. ${ }^{32}$ It has been well documented that synthetic cannabinoids often do not produce molecular ion peaks. ${ }^{7}$ These advancements increase the potential that identifications, or suspected identifications, will result from non-targeted analyses, but without a known molecular mass is not universally applicable to NPSs.

The variation, repeatability, and reproducibility of the GCMS data used for compound identification has not been extensively studied; relevant work is detailed below. It is also worth noting that although HDX has been shown to discriminate small glycan isomers with minute differences ${ }^{24}$, this technique has not, to date, been applied to NPSs. Historically, retention time and RI variation has been investigated intermittently. Temperature programmed RIs from pesticides and fatty acids were shown to be highly reproducible, even after the column length was changed for regular maintenance. ${ }^{33}$ More recent work by Davidson et. $\mathrm{al}^{34}$, assessed the uncertainty of retention times produced from various illicit drugs and compared the interlaboratory results to existing guidelines, like those set forth by the UNODC. ${ }^{34}$ It was shown that the measured uncertainty was smaller than the uncertainty criteria in the guidelines. ${ }^{34}$ However, retention times are not universally comparable, in that, they are dependent on instrument settings. This work also evaluated the inter-laboratory uncertainty of ion \%RAs; the results indicated that the uncertainty was similar to the acceptance criteria, but that the \%RAs are not independently variable within a spectrum, i.e. the uncertainty of ions is not independent of fragmentation pathways. ${ }^{34}$ Little work has evaluated inherent variation within GCMS instruments and across vendor platforms, which, anecdotally, is assumed to be negligible.

With these studies in mind, this body of work had two specific aims focusing on the evaluation of non-target NPS analyses. 
(1) investigate the inherent variation within GCMS instrumentation from different vendors

(2) investigate if HDX-IMS-MS was capable of NPS isomer discrimination.

Understanding the inherent variation in GCMS instrumentation and investigating strategies to limit the variation, provides information about how well drug chemists are identifying true unknown NPS analogues. Next, if isomer discrimination is possible with HDX-IMS-MS then the technique is capable of non-target analyses, albeit with limitations, as all other analytical techniques are subject to.

This first aim was addressed in two studies. Initially, the 'worst case scenario' was evaluated by not conducting instrument tuning and vendor recommended maintenance over an extended time period. Next, the instrument intra- and inter-day variation was evaluated with regular tuning and maintenance. It was also determined if the instrument's autotune passed tuning criteria set forth by the Environmental Protection Agency and investigated if passing this criterion reduced ion \%RA variation. The second aim was addressed in a single study. Six NPSs were initially chosen to determine the propensity of deuterium exchange; next, HDX NPS isomer discrimination was evaluated using three disubstituted aromatic ring isomers (ortho-, meta-, and para-).

\subsection{References}

1. UNODC, Worlds Drug Report 2013. New psychoactive substances: concepts and definitions. Crime, U. N. O. o. D. a., Ed. Vienne, 2013; pp 59-115.

2. UNODC, The challenge of new psychoactive substances. Crime, U. N. O. o. D. a., Ed. United Nations Office on Drugs and Crime: 2013.

3. UNODC, World Drug Report. UNODC, Ed. United Nations Office on Drugs and Crime: 2017. 
4. Zawilska, J. B.; Andrzejczak, D., Next generation of novel psychoactive substances on the horizon- A complex problem to face. Drug and Alcohol Dependence 2015, (157), 1-17.

5. Schifano, F.; Duccio Papanti, G.; Orsolini, L.; Corkery, J. M., Novel psychoactive substance: The pharmacology of stimulants and hallucinogens. Expert Review of Clinical Pharmacology 2016, 9 (7), 943-54.

6. Castaneto, M. S.; Gorelick, D. A.; Desrosiers, N. A.; Hartman, R. L.; Pirard, S.; Huestis, M. A., Synthetic cannabinoids: epidemiology, pharmacodynamics, and clinical implications. Drug and alcohol dependence 2014, 144, 12-41.

7. Namera, A.; Kawamura, M.; Nakamoto, A.; Saito, T.; Nagao, M., Comprehensive review of the detection methods for synthetic cannabinoids and cathinones. Forensic Toxicology 2015, (33), 175-94.

8. German Christopher, L. C.; Fleckenstein, A. E.; Hanson, G. R., Bath salts and synthetic cathinones: an emerging designer drug phenomenon. Life Sciences 2014, 97 (1), 2-8.

9. Zawilska, J. B., " Legal highs"-new players in the old drama. Curr Drug Abuse Rev 2011, $4(2), 122-130$.

10. Katselou, M.; Papoutsis, I.; Nikolaou, P.; Spiliopoulou, C.; Athanaselis, S., AH-7921: the list of new psychoactive opioids is expanded. Forensic Toxicology 2015, 33 (2), 195-201.

11. Schifano, F.; Albanese, A.; Fergus, S.; Stair, J. L.; Deluca, P.; Corazza, O.; Davey, Z.;

Corkery, J.; Siemann, H.; Scherbaum, N.; Farre', M.; Torrens, M.; Demetrovics, Z.; Ghodse, A. H., Mephedrone (4-methylmethcathinone; 'meow meow'): chemical, pharmacological and clinical issues. Psychopharmacology 2011, 214 (3), 593-602.

12. Controlled Substances Act. In 21, States, U., Ed. 1970. 
13. Controlled Substances Act: Treatment of Controlled substance Analogues. In 21, 21 ed.; United States: 1986; Vol. 13B.

14. Ledberg Anders, A., The interest in eight new psychoactive substances before and after scheduling. Drug and Alcohol Dependence 2015, 152, 73-8.

15. Belackova, V.; Pazitny, M.; Drapalova, E.; Martinez, M.; van der Gouwe, D.; Begley, E.; Kidawa, M.; Tomkova, A.; Kmetonynova, D., Assessing the impact of laws controlling the online availability of 25I-NBOMe, AH-7921, MDPV and MXE-outcomes of a semi-automated e-shop monitoring. Drugs: Education, Prevention and Policy 2018, 25 (2), 109-117.

16. Son, C. Y.; Yethiraj, A.; Cui, Q., Cavity hydration dynamics in cytochrome c oxidase and functional implications. Proceedings of the National Academy of Sciences 2017, 114 (42), E8830-E8836.

17. Drugs, S. W. G. f. t. A. o. S., Recommendations version 7.1. Agency, D. E., Ed. 2016; Vol. 2018.

18. Holler, F. J.; Skoog, D. A.; Crouch, S. R., Principles of instrumental analysis. Belmont: Thomson 2007.

19. Gross, J. H., Mass spectrometry: a textbook. Springer Science \& Business Media: 2006.

20. Kostyukevich, Y.; Acter, T.; Zherebker, A.; Ahmed, A.; Kim, S.; Nikolaev, E., Hydrogen/deuterium exchange in mass spectrometry. Mass spectrometry reviews 2018.

21. Majuta, S. N.; Maleki, H.; Kiani Karanji, A.; Attanyake, K.; Loch, E.; Valentine, S. J., Magnifying ion mobility spectrometry-mass spectrometry measurements for biomolecular structure studies. Current Opinion in Chemical Biology 2018, 42, 101-110. 
22. Lee, J.-J.; Park, Y. S.; Lee, K.-J., Hydrogen-deuterium exchange mass spectrometry for determining protein structural changes in drug discovery. Archives of pharmacal research 2015, 38 (10), 1737-1745.

23. Masson, G. R.; Jenkins, M. L.; Burke, J. E., An overview of hydrogen deuterium exchange mass spectrometry (HDX-MS) in drug discovery. Expert opinion on drug discovery 2017, 12 (10), 981-994.

24. Uppal, S. S.; Beasley, S. E.; Scian, M.; Guttman, M., Gas-phase hydrogen/deuterium exchange for distinguishing isomeric carbohydrate ions. Analytical chemistry 2017, 89 (8), 4737 4742.

25. Khakinejad, M.; Kondalaji, S. G.; Maleki, H.; Arndt, J. R.; Donohoe, G. C.; Valentine, S. J., Combining ion mobility spectrometry with hydrogen-deuterium exchange and top-down MS for peptide ion structure analysis. Journal of the American Society for Mass Spectrometry 2014, 25 (12), 2103-2115.

26. Maleki, H.; Karanji, A. K.; Majuta, S.; Maurer, M. M.; Valentine, S. J., Ion Mobility Spectrometry-Mass Spectrometry Coupled with Gas-Phase Hydrogen/Deuterium Exchange for Metabolomics Analyses. Journal of The American Society for Mass Spectrometry 2018, 29 (2), 230-241.

27. Campbell, S.; Rodgers, M.; Marzluff, E. M.; Beauchamp, J., Deuterium exchange reactions as a probe of biomolecule structure. Fundamental studies of gas phase H/D exchange reactions of protonated glycine oligomers with D2O, CD3OD, CD3CO2D, and ND3. Journal of the American Chemical Society 1995, 117 (51), 12840-12854. 
28. Stein, S. E.; Scott, D. R., Optimization and testing of mass spectral library search algorithms for compound identification. Journal of the American Society for Mass Spectrometry 1994, 5 (9), 859-866.

29. Ausloos, P.; Clifton, C.; Lias, S.; Mikaya, A.; Stein, S. E.; Tchekhovskoi, D. V.; Sparkman, O.; Zaikin, V.; Zhu, D., The critical evaluation of a comprehensive mass spectral library. Journal of the American Society for Mass Spectrometry 1999, 10 (4), 287-299.

30. McLafferty, F. W.; Stauffer, D. A.; Loh, S. Y.; Wesdemiotis, C., Unknown identification using reference mass spectra. Quality evaluation of databases. Journal of the American Society for Mass Spectrometry 1999, 10 (12), 1229-1240.

31. Wallace, W. E.; Ji, W.; Tchekhovskoi, D. V.; Phinney, K. W.; Stein, S. E., Mass spectral library quality assurance by inter-library comparison. Journal of The American Society for Mass Spectrometry 2017, 28 (4), 733-738.

32. Moorthy, A. S.; Wallace, W. E.; Kearsley, A. J.; Tchekhovskoi, D. V.; Stein, S. E., Combining fragment-ion and neutral-loss matching during mass spectral library searching: A new general purpose algorithm applicable to illicit drug identification. Analytical chemistry 2017, 89 (24), 13261-13268.

33. Miyagawa, H.; Nakagawa, K.; Kadokami, K., Reproducibility of programmedtemperature retention indices under average linear velocity carrier gas control of GC and GCMS. Chromatographia 2011, 73 (9-10), 953-963.

34. Davidson, J. T.; Lum, B. J.; Nano, G.; Jackson, G. P., Comparison of measured and recommended acceptance criteria for the analysis of seized drugs using Gas ChromatographyMass Spectrometry (GC-MS). Forensic Chemistry 2018, 10, 15-26. 


\section{Chapter 2: Evaluation of the Reproducibility and Repeatability of GCMS Retention Indices and Mass Spectra of Novel Psychoactive Substances.}

Reproduced from Elsevier's Forensic Chemistry journal: Kelly, K.; Bell, S. "Evaluation of the reproducibility and repeatability of GCMS retention indices and mass spectra of novel psychoactive substances." Forensic Chemistry 7 (2018): 10-18.

Permission was obtained from the co-authors and was not required by the journal according to Elsevier's copyright and permission guidelines. The policy is located at the following web address under the personal use clause: https://www.elsevier.com/about/policies/copyright\#

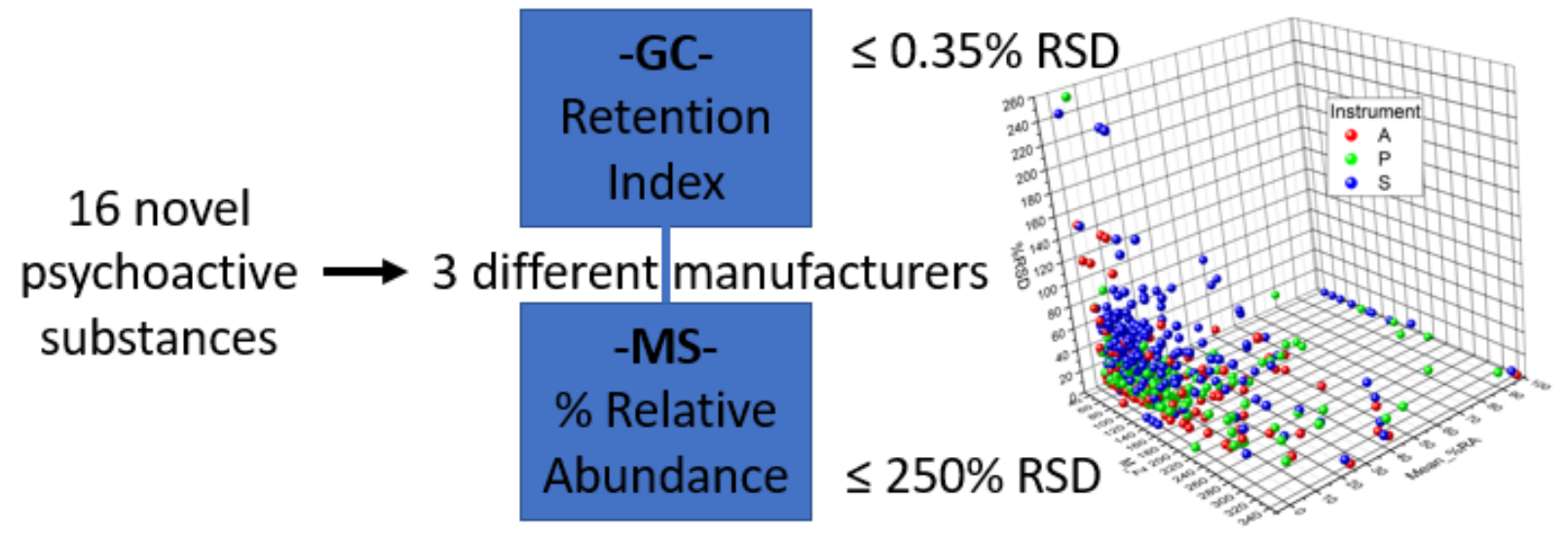

\subsection{Introduction}

Novel psychoactive substances (NPSs) are a family of synthetic compounds designed to mimic the effects of other illicit drugs. ${ }^{1-2}$ The two largest categories of NPSs are synthetic cannabinoids and designer cathinones; other classes include piperazines, tryptamines, and opioid mimics. Although NPSs are currently regulated by various agencies worldwide, these regulations are largely limited by the compound structure. ${ }^{3-6}$ Small side chain substitutions and minor structural changes, which have varying effects on pharmacological activity, allow new analogs to temporarily evade these drug regulations. ${ }^{1-3}$ Amendments, like the Anti-Drug Abuse Act of 1986 in the United States ${ }^{7}$, were created to extend legislation to analogues of specific classifications of controlled substances, so long as these analogues are sold for human consumption. ${ }^{7}$ To 
circumvent amendments similar to this, two strategies have been used. Initially, NPSs were sold as 'legal highs', 'research chemicals', 'plant food', among other names, with various disclaimers such as "not for human consumption" and "for research purposes only". ${ }^{1-2,4,8}$ Later, synthesis of substances with little structural analogy to controlled substances but similar pharmacological properties were pursued. ${ }^{5}$ This legislative limitation has created a revolving door of NPSs where a new analogue is readily available to act as a replacement once another has been scheduled. ${ }^{6,9}$ This cycle has created two problems for the forensic science community- non-availability of standards ${ }^{9}$ and numerous isobaric variants of similar core structures have been synthesized. While the lack of standards may preclude definitive identification for legal proceedings, the ability to identify NPSs is still critical for investigative and intelligence purposes. In the forensic laboratory setting, this implies reliance on comparison with spectra published in databases.

Currently, gas chromatography-mass spectrometry (GCMS) is the most commonly employed instrument for the analysis of seized NPSs in a forensic setting. As such, retention time can be used as a part of the analytical identification scheme, particularly when comparing an unknown to a reference compound. However, with NPSs, standards often are not available and analysts must fall back on mass spectral interpretation and chromatographic behavior to postulate the identification of an unknown. In this context, retention time can be valuable as long as these values are comparable across instruments. A retention index (RI) method was proposed by Kovats more than 50 years ago as a means to do so in an era when MS was not widely available as a GC detector. ${ }^{10}$ The RI of an analyte is the expression of the retention time relative to the two n-alkanes that elute immediately before and after the target analyte. While this necessitates that an n-alkane solution, or "carbon ladder", be analyzed contemporaneously, the advantage is that the RI value is more independent of experimental conditions than retention 
time. Thus, while retention times can vary with column type, temperature program, etc., the RI remains constant within a range of variation. Furthermore, a RI value can be used in an analytical identification scheme by including or excluding potential NPSs. However, to integrate RI in this way, the uncertainty, or variability, of RI values must be established. This was one of the goals of this study.

Several analytical challenges have arisen in the last decade that has revived interest in RIs. The term "non-target" analysis can refer to NPSs as is the case here, or to metabolomics or other "-omics" in which identification of a compound that cannot be confirmed by comparison to reference standards. Instead, identification relies on instrumental data such as mass spectra and retention time. There are two methods for calculating RI depending on the temperature settings of the gas chromatograph (isothermal vs. temperature programmed). Some mass spectral libraries contain RIs, either predicted by modeling or experimental, which can eliminate compounds during an identification search. ${ }^{10-14}$ One recent study investigated the reproducibility of program temperature RIs by varying the chromatographic experimental conditions. ${ }^{15}$ The authors did not report statistical values but indicate that RIs were nearly constant, even if the capillary column was shortened for maintenance, so long as there is constant carrier gas velocity. However, this study was conducted on a single instrument and column and lacked a quantitative metric to describe variability. ${ }^{15}$

Although standards can be purchased and mass spectra can be manually compared, mass spectral fragmentation patterns can also be searched against comprehensive library databases, like SWGDRUG (for seized drugs and NPSs) and NIST (broadly). ${ }^{3,6,9,16}$ However, the pace of standard availability and database updates inevitably lags behind the pace of the introduction of new NPSs. ${ }^{16}$ Even when trustworthy standard spectra are available through these entities, the 
identification of a substance as being one in the database or as something not in the database relies on factors often taken for granted. This study explores two of these underlying and often unappreciated factors- the uncertainty (variability) of RIs across instruments and the variability of mass spectral patterns across instruments. Without knowing these uncertainties, any identification (or elimination) based on comparisons becomes problematic. Recent publications acknowledge the complications of NPS identification in this regard. For example, authors have noted problems distinguishing compounds with complex fragmentation patterns because the pattern loses specificity ${ }^{9}$; other authors have advocated for high resolution mass spectrometry for the identification of new synthetic cannabinoids. ${ }^{17}$ This would certainly be an improvement over typical electron impact MS systems, but high resolution MS does not solve the problem of isobaric compounds commonly seen in the world of NPSs. Thus, to assist investigators in accessing mass spectral comparison, a second goal of this study was to establish how much variation is expected to be observed in mass spectra (in terms of relative ion abundance) within and between instruments over time as a function of column, experimental conditions, and tuning criteria.

Few studies of mass spectral variability were located in the context of non-target compound identification. Alternatives to database searching have been developed for metabolomics data processing. One such cloud based program is the XCMS Online. ${ }^{18}$ Although originally the platform was only capable of two group analysis, the platform continues to be updated and now allows for multiple group and meta-analysis and is capable of the deconvolution of metabolomics data from a number of instrumental designs. ${ }^{19}$ In $2017 \mathrm{Yu}$ et al. ${ }^{20}$ adapted the XCMS input functions to explore electron impact (EI) gas chromatography (GC) MS full-scan mass spectral profiles of trace environmental samples. ${ }^{20}$ Using a boundary regression 
model the authors were able to decrease the analysis uncertainty by identifying and separating peaks likely contributable to noise. ${ }^{20}$ However, the authors used raw mass spectral peak intensities rather than percent relative abundances, which are used for database searching. Peak deconvolution, although shown to decrease analysis uncertainty for metabolomics, may not be easily applicable to forensic drug chemistry analyses.

Initially, the project goals included developing models to generate quantitative estimates of uncertainty in RIs and mass spectral abundances. In this context, uncertainty is defined as the range of values which can reasonably be attributed to the measurand such as RI and percent relative abundance (\%RA) of a given $m / z$ ion. ${ }^{21}$ Several methods are used for estimating measurement uncertainty. ${ }^{21-23}$ Often, measurement uncertainty is closely linked to method validation and derivation of figures of merit. ${ }^{24-26}$ One of the challenges of estimating uncertainty in the context of GCMS and NPSs is how to capture and express variation across instruments and laboratories. For example, if one laboratory uses vendor A's GCMS, vendor B's column, and instrument tuning criteria "C", while a laboratory in another jurisdiction uses completely different materials, estimation of uncertainty necessitates capturing the variation in RI values and mass spectral patterns associated with these different conditions. An uncertainty estimation process requires quantitative data regarding repeatability and reproducibility. Before such an estimate can be made, procedural steps to determine the amount of and minimize the existing variation must be performed.

The goal of this study was to evaluate variation in RI and ion \% RA among and between 16 NPSs on GCMS instruments from three manufacturers using different instrumental settings, columns, tuning criteria, and usage patterns. The descriptive statistics of variables (RIs and mass 
spectral \%RAs) for each compound were collected and evaluated for significant differences at $\alpha=0.05$.

\subsection{Materials and Methods}

\subsubsection{Chemical and Reagents}

LCMS grade purity methanol and hexane obtained from Fisher Chemical (Fair Lawn, NJ) was used for the NPS mixes and the carbon ladder solutions, respectively, as well as solvent blanks. The $\mathrm{C}_{7}-\mathrm{C}_{40}$ ladder standards were obtained from Supelco Analytical (Bellefonte, PA). NPS standards were obtained from Cerilliant (Round Rock, TX) and Cayman Chemical (Ann Arbor, MI).

Three drug mixes containing $\sim 15 \mathrm{ppm}$ of each NPS, one $50 \mathrm{ppm}$ carbon ladder solution, and two solvent blanks (methanol and hexane) were made for each instrument. NPSs were separated into 3 solutions based on retention time- where a minimum of 0.4 minutes separated the compounds; the contents of the solutions are shown in Table 2.1. As this study was not quantitative, the actual concentration of each NPS was approximated and instead the NPS concentrations were adjusted (within $5 \mathrm{ppm}$ ) to ensure adequate GC signal intensity ( $\geq 30 \%$ normalized) for analysis. A low concentration of individual analytes was purposefully targeted, as this is where the most variation is anticipated to exist in mass spectral abundance patterns. 
Table 2.1. NPSs contained in each of the three mixes.

\begin{tabular}{cc}
\hline Mix & Compounds \\
\hline & 2,5-dimethoxy-4-iodoamphetamine \\
Blue & 25I-NBOMe \\
& Amphetamine \\
& Butylone \\
JWH-018 \\
\hline \multirow{4}{*}{ Red } & 1-(3-chlorophenyl)piperazine \\
& 4-fluoroamphetamine \\
& 25C-NBOMe \\
& Benzyl piperazine \\
UR-144 \\
\hline \\
Yellow & 5-MeO-AMT \\
& Dimethyltryptamine \\
& MDMA \\
& Mephedrone \\
& AH-7921
\end{tabular}

\subsubsection{GCMS Instruments}

Prior to analysis, each instrument underwent the regular maintenance of ionization source cleaning, column clipping, and changing the inlet liner. The Agilent (A) was tuned periodically up to 2 times per week while the Perkin Elmer (B) and Shimazdu (C) were tuned once for the duration of the experiment prior to first analysis. All instruments were tuned using the respective autotune feature and perfluorotributylamine (PFTBA) as a tuning compound. The instrument models and method settings for each instrument are shown in Table 2.2. Instruments A and B were used for two and one additional projects, respectively, resulting in an almost 24/7 analysis time for the duration of the study. Instrument $\mathrm{C}$ was used solely for this project resulting in less overall analysis time. For this initial study, a variety of experimental conditions such as columns and settings were purposely selected to encompass the range of conditions typical across forensic laboratories. 
Table 2.2. Model number and method settings for GCMS systems.

\begin{tabular}{ccccc}
\hline & Setting & A & B & C \\
\hline & Model & $7890 \mathrm{~B}$ & Clarus 680 & GC-2010 \\
& Column Type & HP-5 & ZB-5MS & ZB-5MSi \\
\multirow{4}{*}{ GC } & Column L X ID X df $(\mathrm{m} \mathrm{X} \mathrm{mm} \mathrm{X} \mu \mathrm{m})$ & $30 \times 0.25$ X 0.25 & 20 X 0.18 X 0.18 & 30 X 0.25 X 0.25 \\
& Injection Temperature $\left({ }^{\circ} \mathrm{C}\right)$ & 250 & 280 & 270 \\
& Initial Column Temperature $\left({ }^{\circ} \mathrm{C}\right)$ & 100 & 100 & 100 \\
& Final Column Temperature $\left({ }^{\circ} \mathrm{C}\right)$ & 320 & 320 & 305 \\
& Ramp $\left({ }^{\circ} \mathrm{c} / \mathrm{min}\right)$ & 15 & 15 & 10 \\
\hline \multirow{4}{*}{ MS } & $\operatorname{Model}$ & $5977 \mathrm{AMSD}$ & Clarus SQ 8 S & QP2010S \\
& $\mathrm{MS} \mathrm{Source} \mathrm{Temperature}\left({ }^{\circ} \mathrm{C}\right)$ & 250 & 250 & 225 \\
& EM Voltage $(\mathrm{V})$ & 1910 & 1792 & 1080 \\
& $\mathrm{MS} \mathrm{Scan} \mathrm{Range}(\mathrm{m} / z)$ & $40-400$ & $40-400$ & $40-500$ \\
\hline
\end{tabular}




\subsubsection{Experimental Design and Data Analysis}

A complete sample set, analysis order shown in Table 2.3, was then collected on each instrument periodically. A dataset was accepted for analysis if: there was no instrument error during the sample set, all solvent blanks were uncontaminated, and there was adequate GC signal intensity for peaks to be well defined, see Figure 2.1 for a flow chart.

Table 2.3. Complete analysis in order which were performed.

\begin{tabular}{cc}
\hline Position in Analysis & Sample \\
\hline 1 & Hexane solvent blank \\
2 & $\mathrm{C}_{7}-\mathrm{C}_{40}$ ladder solution \\
3 & Hexane solvent blank \\
4 & Methanol solvent blank \\
5 & Red mix \\
6 & Methanol solvent blank \\
7 & Yellow mix \\
8 & Methanol solvent blank \\
9 & Blue mix \\
10 & Methanol solvent blank \\
\hline
\end{tabular}

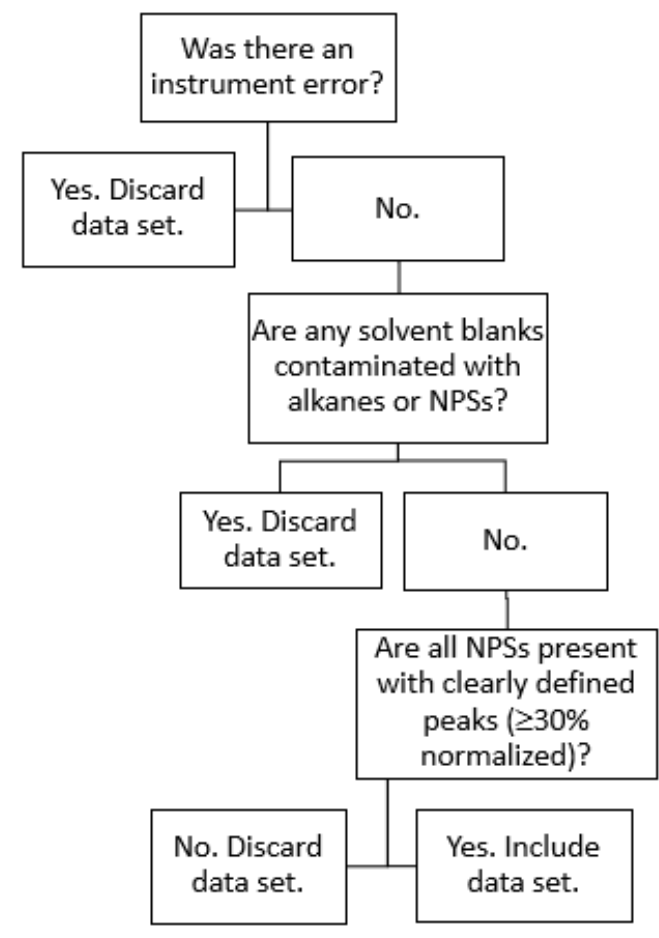

Figure 2.1. Flow chart of how a sample set was determined to be included or discarded. 
For all datasets deemed acceptable, the retention times were recorded and RIs were calculated based on the temperature programming RI equation, Equation 2.1. ${ }^{10}$

$I_{x}=100\left(n+\frac{\left(t_{x}-t_{n}\right)}{\left(t_{N}-t_{n}\right)}\right)$

where $I_{x}$ is the RI of the compound of interest, $n$ is the number of carbon atoms in the alkane eluting just prior to the compound. $t_{x}$ is the retention time of the compound of interest, $t_{n}$ is the retention time of the alkane eluting just prior to the compound of interest, and $t_{N}$ is the retention time of the alkane eluting just after the compound of interest.

To obtain the representative mass spectrum for each peak, the spectra across the middle of the peak (as estimated using full-width half-max) were averaged and the background from before and after the peak was subtracted. This procedure ensured that the mass spectrum subjected to further processing was not biased based on where it was observed across the elution profile. From this spectrum, the $\%$ RA and $\mathrm{m} / \mathrm{z}$ value of the 20 most abundant mass spectra peaks were recorded. The datasets for each NPS and all instruments were then combined for data analysis by transcription into Excel data files. Data entry and transcription was independently checked for each batch of samples analyzed, extracted, and organized into spreadsheets.

Once all datasets were combined the average \%RA for each of the most abundant mass spectra peaks was calculated. If the three-system \%RA average was below $2 \%$ the peak was removed from the data set to prevent peaks associated with noise from being included in the statistical analysis. Descriptive statistics and one-way ANOVA analysis was conducted in OriginLab® (OriginLab Corporation, Northampton, MA).

\subsection{Results and Discussion}


The number of acceptable datasets from each instrument was A ( $n=9), B(n=14)$, and, C $(n=6)$ for a total sample set of $n=29$. The percentage of datasets deemed acceptable was $82 \%$, $74 \%$, and $60 \%$ for instruments A, B, and C, respectively. The majority of the 'unacceptable' datasets were due to missed injections, where a single compound mix or the carbon ladder were missing, or due to an autosampler error and all three compound mixes were not collected. Instrument B operated for the longest time span of $\sim 12$ weeks, whereas instrument A and C operated for $\sim 8$ weeks and 4 weeks, respectively.

\subsubsection{Retention Indices}

The RIs for 4-fluoroamphetamine and amphetamine on instrument $\mathrm{C}$ could not be calculated because on the columns used, the corresponding n-alkane co-eluted with the solvent. The descriptive statistics, shown in Table A1, details the variation and repeatability of RIs for each instrument. The RSD across all systems and compounds was $\leq 0.35 \%$, as expected, which emphasizes the potential value of incorporating RI in any cross-identification scheme.

Interestingly, a means comparison of the RIs (Figure 2.2) showed significant differences between the three systems despite the small overall \%RSD. As seen in Figure 2.2, statistically significant differences, shown in red, were identified in 39 of the 44 comparisons. One possibility is that differences in the column aging and degradation generated variation between instruments. A second possibility is that the variation resulted from differences in temperature programming and column type. Despite the statistical differences and regardless of the cause, the largest difference in RI between any two instruments for a single compound was 37.0 RI units. This reiterates the utility of incorporating RIs in non-targeted analysis. For example, if a mass spectral library search returns 50 potential matches, a comparison of RIs would be able to 
eliminate potential matches because the RIs are outside of a determined uncertainty range. Identification of this range will require further studies.

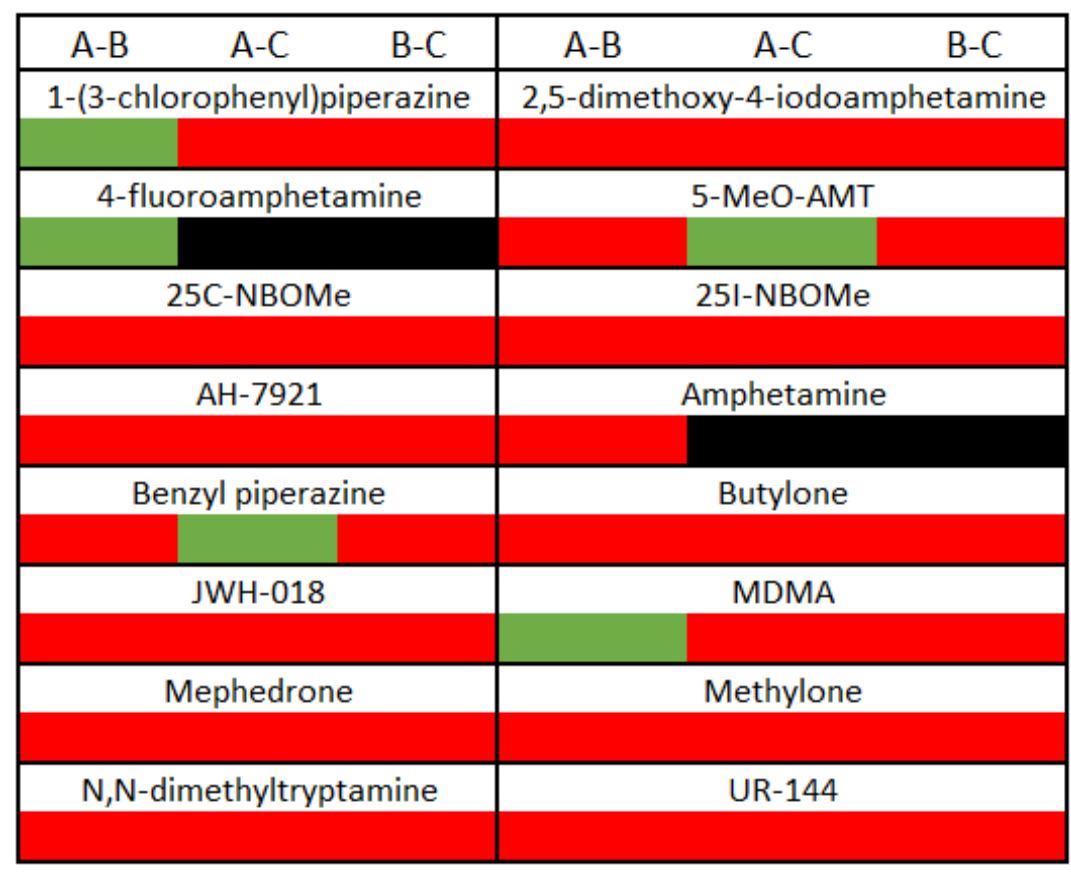

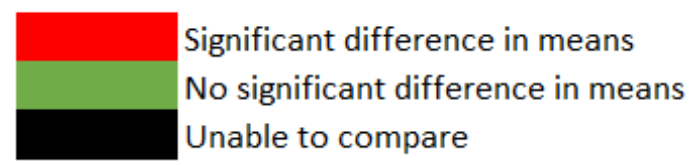

Figure 2.2. Means comparison of RIs for all NPSs.

\subsubsection{Mass Spectra}

\subsection{2a Mean Relative Abundance Comparisons}

The number of specific $\mathrm{m} / \mathrm{z}$ ions included in the statistical analysis for each NPSs ranged from 10 to 17 depending upon the "richness" (number of fragment peaks above 2\% RA three system average) of the spectrum. An example of a feature-poor and feature-rich mass spectra are shown in Figures 2.3a and 2.3b, respectively. Ions of 100\% RA (base peak) were included only if the selected peak was not consistently the most abundant; i.e. $100 \%$ in one instance and $95 \%$ in another. Alternating base peaks occurred with three NPSs: JWH-018, 5-MeO-AMT and UR144. As will be discussed later, this type of variation can create a cascading effect across all 
associated \%RAs. If a given $\mathrm{m} / \mathrm{z}$ is always $100 \%$, the variation is zero; when the base peak changes, variation increases across all peaks because the basis $(\mathrm{m} / \mathrm{z}$ identity) of the \%RA changes.

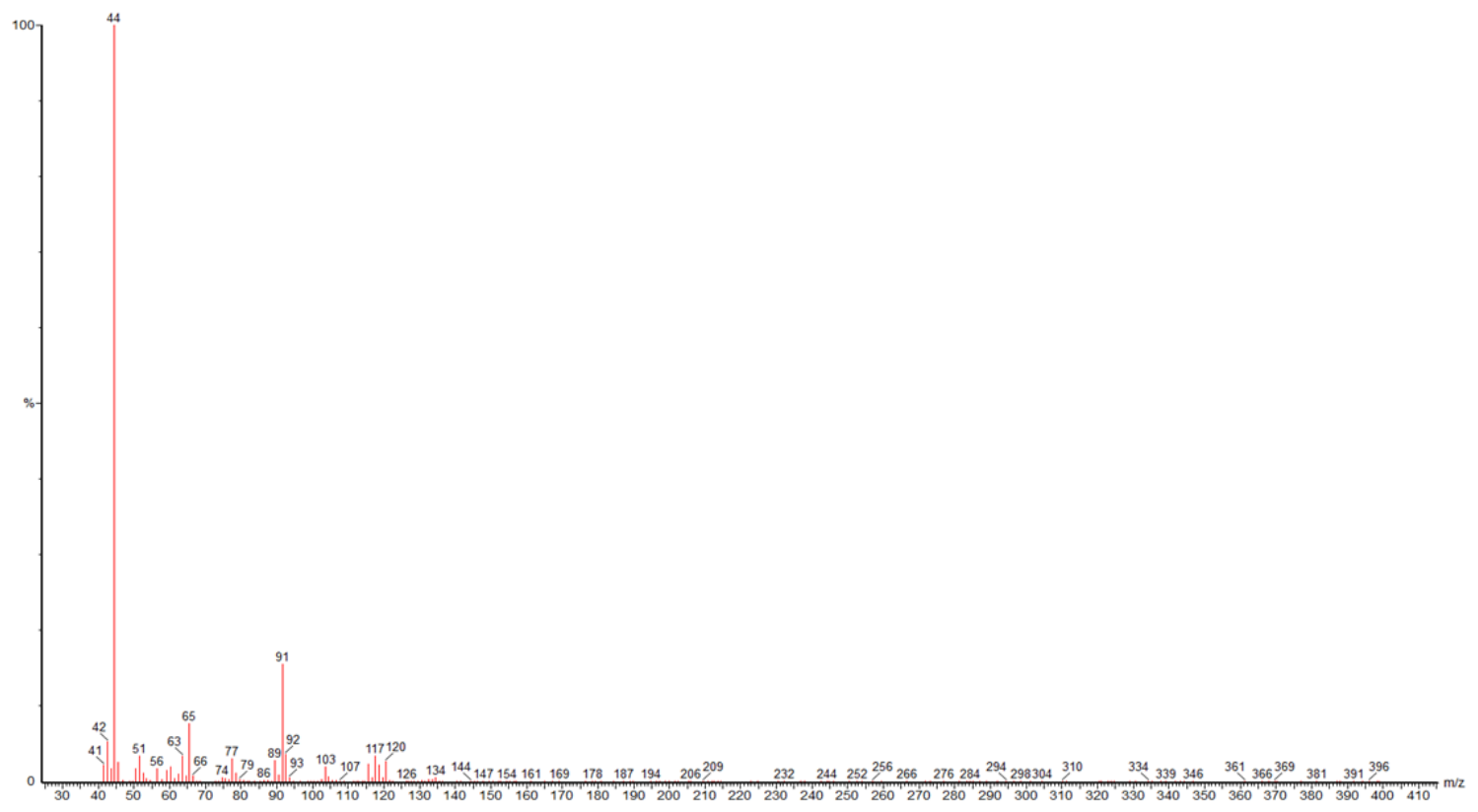

Figure 2.3a. Example of feature-poor spectra (amphetamine).

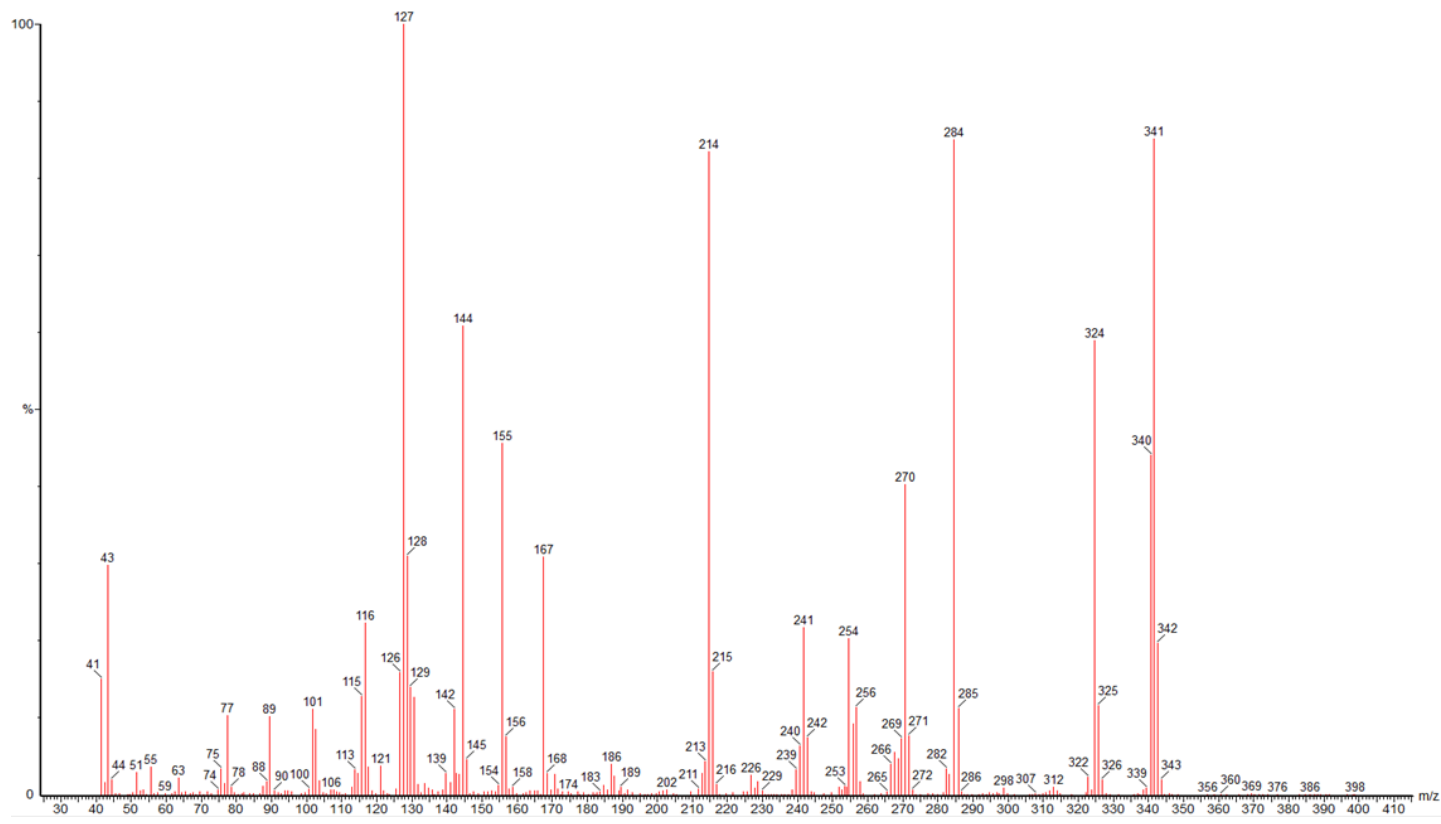

Figure 2.3b. Example of feature-rich spectra (JWH-018). 
Prior to data collection, two hypotheses were developed regarding variation, as measured by $\% \mathrm{RSD}$, in mass spectral \%RAs. The first was that the variation would increase for ions on the extremes of the instrument's scanning range capability. If this were the case, a plot of \% RA as a function of $\mathrm{m} / \mathrm{z}$ value would be a parabolic shape; this pattern was not observed. The second hypothesis was that the \%RSD would increase as the \%RA decreased; i.e., the variation in an $\mathrm{m} / z$ peak of $2 \%$ RA would be expected to be greater than that of an $m / z$ peak with a RA of $60 \%$. This would result in a pattern similar to an exponential decay function with a large decay constant. When the experimental data was plotted (Figure A1a), this generalized pattern plot was observed, but was not sufficiently consistent to fit. Differences between the data from the three systems was evident- instrument $\mathrm{C}$ did not fit the exponential decay pattern as consistently as instrument A and B (Figure A1b).

A three-dimensional plot of $\mathrm{m} / \mathrm{z}, \% \mathrm{RA}$, and $\% \mathrm{RSD}$ and a projection plot for data from all three instruments are shown in Figures A2 and A3, respectively. Apparent outliers were seen in Figure A2 (boxed region). These points represent $m / z$ values present at RAs $>>2$ in two instruments but at a much lower and inconsistent \% RA in the third instrument. Because the three-system average \%RA of these $\mathrm{m} / \mathrm{z}$ values exceeded $2 \% \mathrm{RA}$, the values met the criteria discussed above and were not removed from the dataset in the initial screening. This produced the high \% RSDs observed. In addition, instruments $\mathrm{A}$ and $\mathrm{C}$ had more data points with $>100 \%$ \%RSD than instrument B. These instruments had fewer datasets, which may have attributed to the increased frequency of data points with high variation. To determine if averaging the \%RA of ions over the course of the study was obscuring predictor trends, the \%RA of ions from featurepoor and feature-rich compounds were plotted as a function of time. No recognizable pattern was 
elucidated from the data. Descriptive statistics for the top five ions of select NPSs are detailed in

Table A2 and were considered representative of the complete dataset.

An exhaustive means comparison analysis was performed for all NPSs, all $m / z$ values retained based on the criteria described earlier, and as obtained on all three instruments. The results are summarized in Figure 2.4. Instruments A and B most frequently had no significant difference in means (shown in green), but beyond that, little can be said with confidence other than the means differences between instruments are common with no discernable pattern.

\begin{tabular}{|c|c|c|c|c|c|c|c|c|}
\hline Ion & $A-B \quad A-C \quad B-C$ & Ion & $A-B$ & $\mathrm{~A}-\mathrm{C}$ & Ion & $A-B \quad A-C \quad B-C$ & Ion & $\mathrm{A}-\mathrm{B} \quad \mathrm{A}-\mathrm{C} \quad \mathrm{B}-\mathrm{C}$ \\
\hline \multicolumn{2}{|c|}{ 1-(3-chlorophenyl)piperazine } & \multicolumn{3}{|c|}{ 2,5-dimethoxy-4-iodoamphetamine } & 4-fluoroa & imphetamine & \multicolumn{2}{|c|}{ 5-MeO-AMT } \\
\hline 156 (most) & & 278 (most) & & & 109 (most) & & 161 (most) & \\
\hline 196 & & 77 & & & 83 & & 44 & \\
\hline 56 & & 91 & & & 42 & & 160 & \\
\hline 138 & & 263 & & & 57 & & 146 & \\
\hline 111 & & 42 & & & 107 & & 117 & \\
\hline \multicolumn{2}{|c|}{ 25C-NBOMe } & \multicolumn{3}{|c|}{ 25I-NBOMe } & \multicolumn{2}{|c|}{$\mathrm{AH}-7921$} & \multicolumn{2}{|c|}{ Amphetamine } \\
\hline 150 (most) & & 150 (most) & & & 127 (most) & & 91 (most) & \\
\hline 91 & & 91 & & & 173 & & 65 & \\
\hline 122 & & 122 & & & 145 & & 42 & \\
\hline 77 & & 77 & & & 59 & & 51 & \\
\hline 65 & & 65 & & & 44 & & 63 & \\
\hline \multicolumn{2}{|c|}{ Benzyl piperazine } & \multicolumn{3}{|c|}{ Butylone } & \multicolumn{2}{|c|}{ JWH-018 } & \multicolumn{2}{|c|}{ MDMA } \\
\hline 134 (most) & & 70 (most) & & & 341 (most) & & 77 (most) & \\
\hline 56 & & 42 & & & 284 & & 56 & \\
\hline 65 & & 57 & & & 214 & & 135 & \\
\hline 176 & & 65 & & & 127 & & 51 & \\
\hline 42 & & 149 & & & 324 & & 42 & \\
\hline \multicolumn{2}{|c|}{ Mephedrone } & \multicolumn{3}{|c|}{ Methylone } & $\mathrm{N}, \mathrm{N}$-dimet & thyltryptamine & \multicolumn{2}{|c|}{ UR-144 } \\
\hline 91 (most) & & 56 (most) & & & 130 (most) & & 214 (most) & \\
\hline 56 & & 65 & & & 42 & & 144 & \\
\hline 65 & & 149 & & & 77 & & 43 & \\
\hline 119 & & 63 & & & 143 & & 296 & \\
\hline 42 & & 121 & & & 115 & & 41 & \\
\hline
\end{tabular}

Significant difference in means

No significant difference in means

Figure 2.4. Means comparison of \%RA of five most abundant ions for all NPSs. Ions are in decreasing order of abundance. Base peak only included if it alternated between two or more ion values during the study.

Select mass spectra from various NPS classes with means comparison box charts for the top three most abundant ions are shown in Figures 2.5, 2.6, and A4-A8. The range, as illustrated 
in the box plots, indicated that instrument A showed the least within instrument variation, which is postulated to be due to the increased tuning frequency compared to the other two. The range for instrument $\mathrm{C}$, on the other hand, consistently showed the largest within instrument variation. It is possible that this large variation may be caused, in part, by the small number of datasets from this instrument included in the statistical analysis. For feature-poor spectra the amount of within instrument variation for instrument B was between A and C, shown in Figures 2.5a-c. However, for feature-rich spectra (Figures 2.6a-c) the range is inconsistent, i.e. occasionally smaller than instrument A or larger than instrument $\mathrm{C}$. This was due to an irreproducible base peak, discussed in detail below.

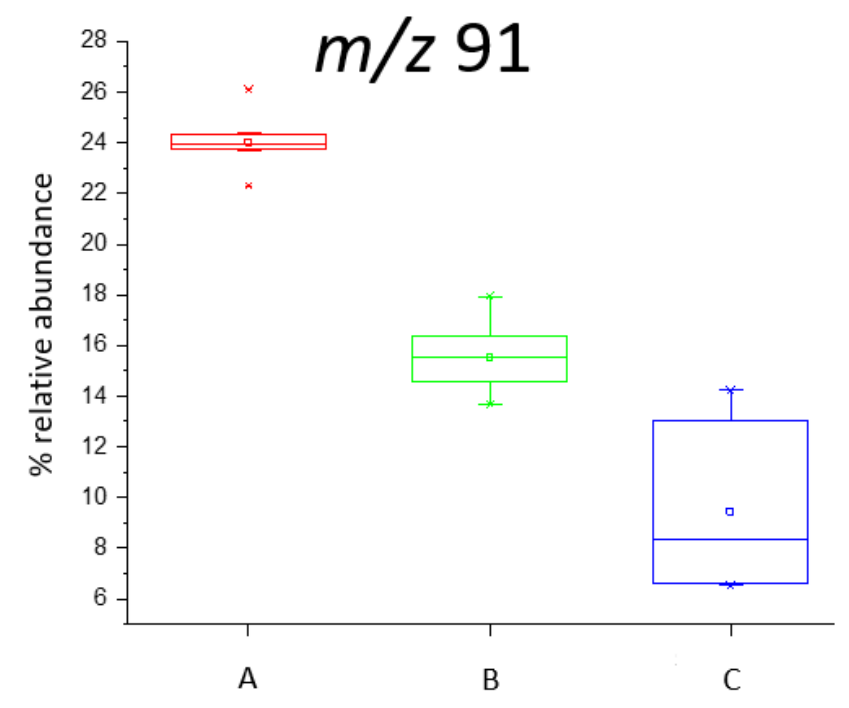

Figure 2.5a. Amphetamine box means comparison chart for $m / z 91$. Most abundant ion. Note the y-axis scale change. 


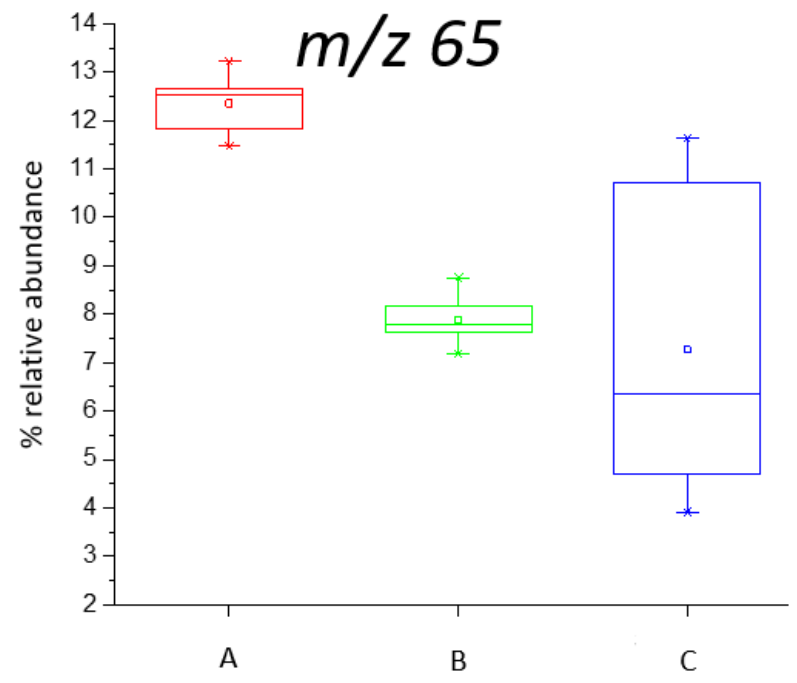

Figure 2.5b. Amphetamine box means comparison chart for $m / z, 65$. Second most abundant ion. Note the y-axis scale change.

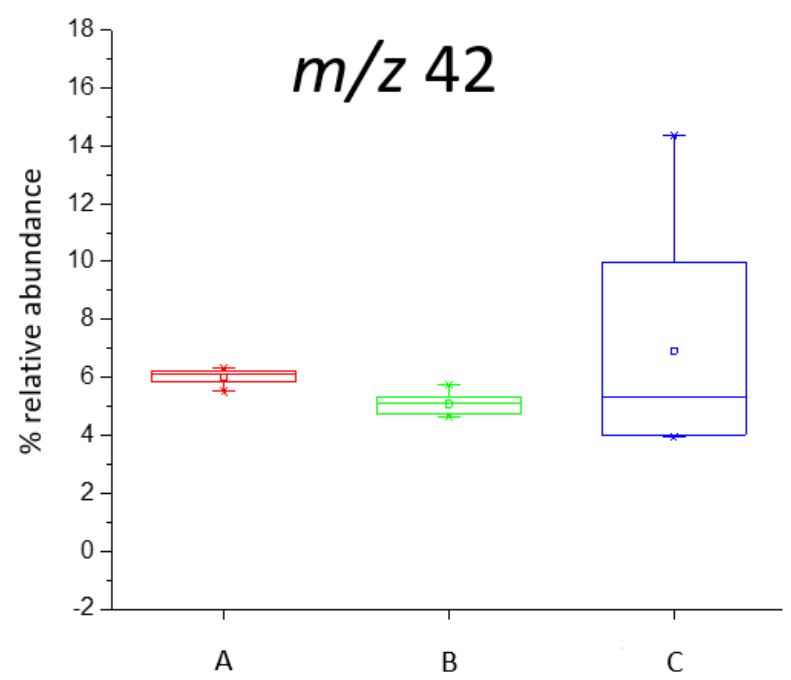

Figure 2.5c. Amphetamine box means comparison chart for $m / z, 42$. Third most abundant ion. Note the y-axis scale change. 


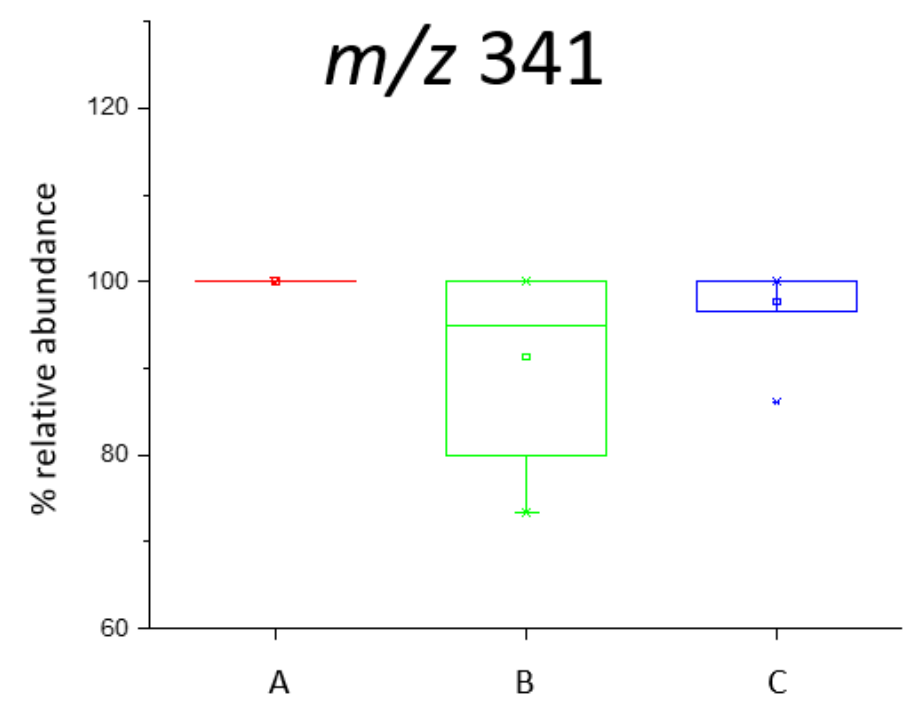

Figure 2.6a. JWH-018 box means comparison chart for $m / z 341$. Most abundant ion. Note the $y$-axis scale change.

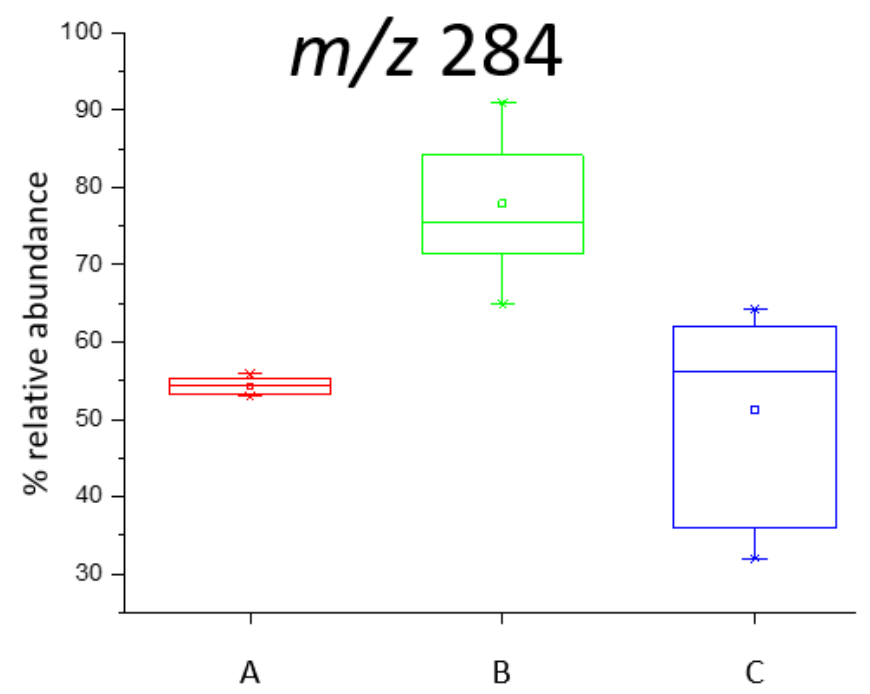

Figure 2.6b. JWH-018 box means comparison chart for $m / z 284$. Second most abundant ion. Note the y-axis scale change. 


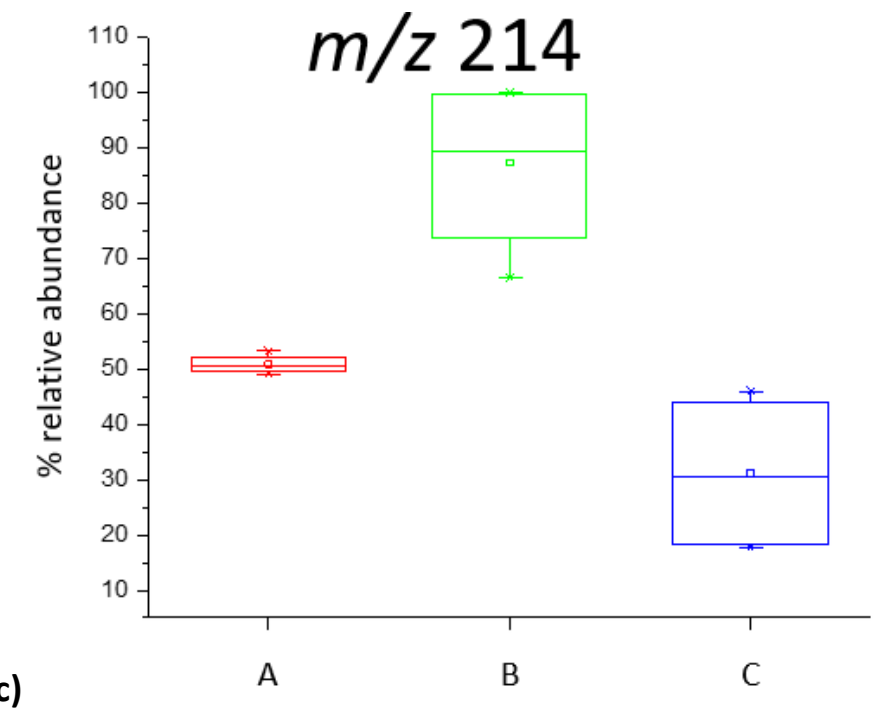

Figure 2.6c. JWH-018 box means comparison chart for $m / z 214$. Third most abundant ion. Note the y-axis scale change.

\subsection{2b Irreproducible Base Peaks}

Three instances of irreproducible base peaks are discussed in detail. The first situation occurred on a single instrument, where the base peak alternated between multiple ions on a single instrument. Alternating base peaks on a single instrument are particularly troublesome in that a ripple effect impacts all related \%RA values. When the base peak is stable, the denominator of the \%RA calculation is a constant; when the base peak changes, the denominator is also a variable. This essentially changes the identity of the \%RA calculations, which causes an increase in the variability of all other \%RAs. The second instances of irreproducible base peak changes are considered anomalies and are discussed below. The final instance of an irreproducible base peak occurred with 5-MeO-AMT. This base peak was inconsistent between instruments, where each instrument produced a single base peak but the base peaks were not necessarily the same. The "ripple" effect was not present in this case because the identity of the $\%$ RA calculations did not change for any one instrument. Each situation is important to discuss in detail as either scenario could hinder proper identification of the NPS. 
The first instance of an alternating base peak occurred with JWH-018, where the base peak altered between $\mathrm{m} / \mathrm{z}$ values of $341,214,127$, and 43 . The peak at $\mathrm{m} / \mathrm{z} 341$ was most frequently the base peak across the three instruments and was the only base peak in the spectra obtained on instrument A. Instrument B's base peak alternated between $m / z 341, m / z 214$, and $m / z$ 127. These ions were present at a RA of $70-100 \%$ in all datasets associated with instrument B. These four ions were present in instruments $\mathrm{A}$ and $\mathrm{C}$ at RAs of 40-60\%. This observation is significant given that instrument B was tuned once but produced the most variable base peak, while instrument A produced a single base peak but was tuned most frequently. Clearly, tuning frequency is a critical factor in variability of $m / z \%$ RA values.

The next instances of alternating base peaks occurred on instrument $\mathrm{C}$ for both JWH-018 and UR-144. Instrument $C$ was the only instrument to produce $m / z 43$ as a base peak for JWH018. This ion was not present in the 20 most abundant ions on instrument A but was present in data associated with systems B and C. The RA ranged from $20-40 \%$ on instruments B and C with the exception of the final two C datasets where the RA was recorded at $78 \%$ and $100 \%$. Instrument $\mathrm{C}$ was the only instrument to produce $\mathrm{m} / \mathrm{z} 43$ as a base peak and it only occurred once. A similar situation occurred with a base peak change for the compound UR-144; the mass spectra with box charts is shown in Figure A7. $\mathrm{m} / \mathrm{z}, 214$ was the base peak for all datasets except the final C dataset where $m / z, 41$ was. This ion had a RA of $5-20 \%$ on instruments $\mathrm{A}, \mathrm{B}$, and C with the exception of the final two C datasets where the RA was recorded at $92 \%$ and $100 \%$. It is unclear what caused the base peak change for the JWH-018 and UR-144 instrument C datasets. These base peak changes are considered anomalies.

The final instance of an irreproducible base peak occurred with 5-MeO-AMT, but in this case, the ripple effect was not observed (Figure A8). The base peak of 5-MeO-AMT for 
instruments $\mathrm{A}$ and $\mathrm{B}$ was $\mathrm{m} / \mathrm{z}, 161$; however, the RA of $\mathrm{m} / \mathrm{z}, 161$ on instrument $\mathrm{C}$ ranged from 20$60 \%$. The base peak for all datasets associated with instrument $C$ was $m / z$ 44. The RA of this ion was approximately $50 \%$ for instrument $\mathrm{A}$, but ranged from $50-90 \%$ for instrument $\mathrm{B}$. The high \%RA observed on instrument B indicates that it may have been possible for the base peak to alternate between from $\mathrm{m} / \mathrm{z}, 161$ to $\mathrm{m} / \mathrm{z} 44$ on this instrument. Had this alternation occurred, the identity of the \%RA calculations would have changed and the "ripple" effects that were seen with JWH-018 would have been present. This issue clearly shows where the use of RI would be of value for identification purposes. Irreproducible base peaks, either between instruments or on a single instrument, may hinder identification of NPSs by preventing mass spectral fragmentation patterns to be effectively compared. RIs were shown to be reproducible and repeatable and provide an additional means of data comparison for compound identification.

\subsection{Conclusion and Next Steps}

The variation, repeatability, and reproducibility of RIs and \%RAs of mass spectra fragmentation peaks were investigated across three instrument systems. There were statistically significant differences in the means of RIs between instruments. However, the overall RSD was $<0.35 \%$ and the largest difference in means was shown to be 37.0 RI units. This demonstrates that the RI is sufficiently reproducible to be used as part of compound identification or, more likely, to eliminate potential identifications during non-targeted analysis. This ability could be useful in the context of isobaric compounds in which the spectral patterns are indistinguishable. Based on the data here, the difference between an experimentally obtained RI should fall within \pm approximately $37 \mathrm{RI}$ units of the known or reference value.

The $\%$ RAs of mass spectra showed variation ranging from $<1.0 \%$ to $\sim 250 \%$ RSD on individual instruments. The differences in variation on instrument systems is postulated to be 
linked to tuning criteria and frequency. The most frequently tuned instrument, instrument A, was shown to consistently have the smallest \% RA variation of the three instruments. Instrument B, which was tuned once prior to first analysis, had the most irreproducible base peak for JWH-018. Although the cause of the alternating base peak is currently unclear, it is possible that increased tuning frequency would reduce the number of alternations and improve the variation of ion \% RA on this system.

The findings of this study clearly show that mass spectral patterns vary significantly between instruments and that a primary factor is detector tuning. Thus, when comparing spectral patterns of non-target compounds (no reference standards), this variability can be critical. Variation was also shown to be linked to the value of the \%RA; lower abundance ions, commonly produced in feature-poor spectra like amphetamine, provide a wide range of variation from $2-25 \%$ RSD. On the other hand, ions with > 20\% RA typically never exceed 20\% RSD. Intra-system variation was shown to be dependent on reproducibility of the base peak ion, which was problematic for feature-rich spectra, like the spectrum produced by JWH-018. RIs can be used as filtering information but will require that such data be generated. This is not current practice in most forensic laboratories.

Two avenues are being explored based on the findings of this work- tuning frequency and tuning check compounds. Tuning check compounds are introduced into a tuned instrument and checked against stringent $\mathrm{m} / \mathrm{z}$ abundance criteria. If not met, the tune is adjusted until a pass is obtained. This is not a new concept and was first widely applied starting in the 1980s associated with analytical methods promulgated by the Environmental Protection Agency. The use of tuning check compounds coupled to frequent tuning (daily or before every batch) is currently being evaluated as a method to reduce spectral variation. The check mix being studied also 
includes a RI check which is being evaluated as a method to adjust for the small changes in RI that were identified in this work. It is the hope that a reasonable and easily adopted protocol for using the methodology can be developed for use in NPSs analyses in the forensic setting.

\subsection{Funding and Acknowledgements}

This work was supported by the United States Department of Commerce, National Institute of Standards and Technology Award \# 70NANB16H105. The authors wish to acknowledge the efforts of undergraduate researchers who tirelessly collected, collated, and double-checked data: James Maxella, Megan Messet, and Victoria Zeigler.

\subsection{References}

1. Zawilska, J. B.; Andrzejczak, D., Next generation of novel psychoactive substances on the horizon- A complex problem to face. Drug and Alcohol Dependence 2015, (157), 1-17.

2. Schifano, F.; Duccio Papanti, G.; Orsolini, L.; Corkery, J. M., Novel psychoactive substance: The pharmacology of stimulants and hallucinogens. Expert Review of Clinical Pharmacology 2016, 9 (7), 943-54.

3. Namera, A.; Kawamura, M.; Nakamoto, A.; Saito, T.; Nagao, M., Comprehensive review of the detection methods for synthetic cannabinoids and cathinones. Forensic Toxicology 2015, (33), 175-94.

4. Katselou, M.; Papoutsis, I.; Nikolaou, P.; Spiliopoulou, C.; Athanaselis, S., AH-7921: the list of new psychoactive opioids is expanded. Forensic toxicology 2015, 33 (2), 195-201.

5. German, C. L.; Fleckenstein, A. E.; Hanson, G. R., Bath salts and synthetic cathinones: an emerging designer drug phenomenon. Life sciences 2014, 97 (1), 2-8. 
6. Zuba, D., Identification of cathinones and other active components of 'legal highs' by mass spectrometric methods. TrAC Trends in Analytical Chemistry 2012, 32, 15-30.

7. Controlled Substances Act. In Treatment of controlled substance analogues, United States, 1986; Vol. 21.

8. Zawilska, J. B., " Legal highs"-new players in the old drama. Curr Drug Abuse Rev 2011, $4(2), 122-130$.

9. Elie, M. P.; Elie, L. E.; Baron, M. G., Keeping pace with NPS releases: fast GC-MS screening of legal high products. Drug Test. Anal. 2013, 5 (5), 281-290.

10. Babushok, V., Chromatographic retention indices in identification of chemical compounds. TrAC Trends in Analytical Chemistry 2015, 69, 98-104.

11. Garkani-Nejad, Z.; Karlovits, M.; Demuth, W.; Stimpfl, T.; Vycudilik, W.; Jalali-Heravi, M.; Varmuza, K., Prediction of gas chromatographic retention indices of a diverse set of toxicologically relevant compounds. Journal of Chromatography A 2004, 1028 (2), 287-295.

12. Barnes, B. B.; Wilson, M. B.; Carr, P. W.; Vitha, M. F.; Broeckling, C. D.; Heuberger, A. L.; Prenni, J.; Janis, G. C.; Corcoran, H.; Snow, N. H., “Retention projection” enables reliable use of shared gas chromatographic retention data across laboratories, instruments, and methods. Analytical chemistry 2013, 85 (23), 11650-11657.

13. Strehmel, N.; Hummel, J.; Erban, A.; Strassburg, K.; Kopka, J., Retention index thresholds for compound matching in GC-MS metabolite profiling. Journal of Chromatography B 2008, 871 (2), 182-190.

14. Linden, R.; Feltraco, L. L.; Comerlato, L. C.; Kellermann, E.; Antunes, M. V., Computer assisted substance identification in systematic toxicological analysis: New life for old methods? Forensic science international 2010, 202 (1), e53-e60. 
15. Miyagawa, H.; Nakagawa, K.; Kadokami, K., Reproducibility of ProgrammedTemperature Retention Indices under Average Linear Velocity Carrier Gas Control of GC and GC-MS. Chromatographia 2011, 73 (9-10), 953-963.

16. Temerdashev, A. Z.; Grigor, I. M.; Rybal chenko, I. V., Evolution of new narcotic substances and methods of their determination. Journal of Analytical Chemistry 2014, 69 (9), 817-44.

17. Castaneto, M. S.; Wohlfarth, A.; Desrosiers, N. A.; Hartman, R. L.; Gorelick, D. A.; Huestis, M. A., Synthetic cannabinoids pharmacokinetics and detection methods in biological matrices. Drug metabolism reviews 2015, 47 (2), 124-174.

18. Tautenhahn, R.; Patti, G. J.; Rinehart, D.; Siuzdak, G., XCMS Online: a web-based platform to process untargeted metabolomic data. Analytical chemistry 2012, 84 (11), 50355039.

19. Gowda, H.; Ivanisevic, J.; Johnson, C. H.; Kurczy, M. E.; Benton, H. P.; Rinehart, D.; Nguyen, T.; Ray, J.; Kuehl, J.; Arevalo, B., Interactive XCMS Online: simplifying advanced metabolomic data processing and subsequent statistical analyses. Analytical chemistry 2014, 86 (14), 6931-6939.

20. Yu, M.; Hou, X.; Liu, Q.; Wang, Y.; Liu, J.; Jiang, G., Evaluation and reduction of the analytical uncertainties in GC-MS analysis using a boundary regression model. Talanta 2017, $164,141-147$.

21. Saffaj, T.; Ihssane, B.; Jhilal, F.; Bouchafra, H.; Laslami, S.; Sosse, S. A., An overall uncertainty approach for the validation of analytical separation methods. Analyst 2013, 138 (16), 4677-4691. 
22. Shegunova, P.; Bercaru, O.; Sejerøe-Olsen, B., Estimation of measurement uncertainty in organic analysis: two practical approaches. Accreditation and Quality Assurance 2008, 13 (1), $11-18$.

23. Konieczka, P.; Namieśnik, J., Estimating uncertainty in analytical procedures based on chromatographic techniques. Journal of Chromatography A 2010, 1217 (6), 882-891.

24. Lim, H. S.; Hwang, J. Y.; Im Kim, J.; Choi, J. C.; Kim, M., Validation and measurement uncertainty evaluation of the GC-MS method for the direct determination of hexamethylenetetramine from foods. Food Science and Biotechnology 2016, 25 (5), 1305-1311.

25. Bettencourt da Silva, R. J.; Silveira, D. M.; Camões, M. F. G.; Borges, C. M.; Salgueiro, P. A., Validation, Uncertainty, and Quality Control of Qualitative Analysis of Tear Gas Weapons by Gas Chromatography-Mass Spectrometry. Analytical Letters 2014, 47 (2), 250-267.

26. Eller, S. C. W. S.; Flaiban, L. G.; Paranhos, B. A. P. B.; Costa, J. L.; Lourenço, F. R.; Yonamine, M., Analysis of 11-nor-9-carboxy- $\Delta 9$-tetrahydrocannabinol in urine samples by hollow fiber-liquid phase microextraction and gas chromatography-mass spectrometry in consideration of measurement uncertainty. Forensic Toxicology 2014, 2 (32), 282-291. 


\section{Chapter 3: The Effect of Mass Spectrometry Tuning Frequency and Criteria on Ion Relative Abundances of Cathinones and Cannabinoids}

Reproduced from Elsevier's Forensic Chemistry journal: Kelly, K.; Brooks, S.; Bell, S. “The effect of mass spectrometry tuning frequency and criteria on ion relative abundances of cathinones and cannabinoids." Forensic Chemistry 12 (2019): 58-65.

Permission was obtained from the co-authors and was not required by the journal according to Elsevier's copyright and permission guidelines. The policy is located at the following web address under the personal use clause: https://www.elsevier.com/about/policies/copyright\#

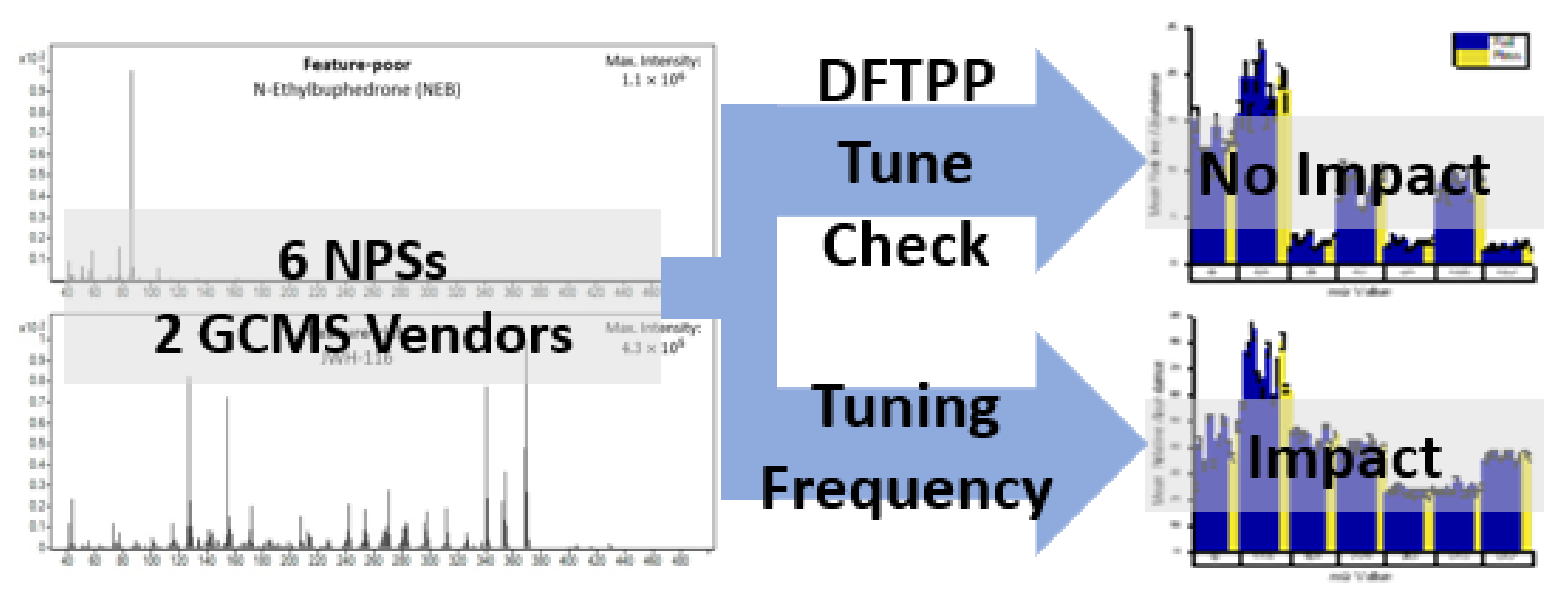

\subsection{Introduction}

Electron impact gas chromatography mass spectrometry (GCMS) is the key instrument for the analysis of seized drugs in a forensic setting. ${ }^{1}$ This technique utilizes retention times and mass spectral fragmentation patterns, typically compared against a reference standard, to confirm the structural identity of compounds. Developing new reference standards is a complex, timeconsuming process, described in detail elsewhere ${ }^{2}$; this process can only be started once a compound has been fully characterized. The rapid introduction of new novel psychoactive substances (NPSs) means there is initially no reference standard available to compare against case sample data. This lack of standards creates a unique issue because many isobars and isomeric derivatives have been identified and can produce nearly identical mass spectra. Without 
reference standards for manual comparison, forensic laboratories rely on non-targeted library searches of mass spectral databases to postulate a tentative identification. ${ }^{3}$ In a library search, the percent relative abundance (\%RA) of all $\mathrm{m} / \mathrm{z}$ values in the mass spectra of an unknown analyte are compared to those of known mass spectra within a library. This yields a list of potential matches along with a score that characterizes the match quality. Verified identification of the new analog and a certified reference standard are required for the updating library databases with new compounds; the forensic community relies heavily on two databases: Scientific Working Group for the Analysis of Seized Drugs (SWGDRUG) for illicit compounds and National Institute of Standards and Technology (NIST) for broad searches. ${ }^{1,3-5}$ Another useful library in seized drug analysis, particularly for NPSs, is the Wiley Mass Spectra of Designer Drugs (Scientific Instrument Services, Ringoes NJ), which contains more than 20,000 compounds related to NPSs.

A critical and often unappreciated consideration is the inherent uncertainty in the \% RA of the $m / z$ values upon which identifications are proposed. Anecdotally, it appears as if intrinsic variability is assumed to be minimal on modern instruments utilizing vendor standardized parameter tuning (i.e., autotuning). However, a recent publication by our group demonstrated that this variation can be significant, up to $250 \% \mathrm{RSD}$, and as such must be taken into consideration when deciding tentative identifications. The previous study examined variation of \%RAs for 16 NPSs analyzed on three instruments from different vendors. Time between tuning was one of several variables in this study. The results suggested that tuning frequency, as an experimental variable, was the most important factor relating to \% RA variation. ${ }^{6}$

The GCMS instruments used in process analytical chemistry, including seized drug analysis, utilize a tuning operation, referred to as autotune or tuning, in which PFTBA 
(perfluorotributylamine) is bled into the mass spectrometer source. Specific fragment ions are used to calibrate multiple component settings of the detector. Typically, voltages applied to lenses are adjusted to optimize the abundance and ion ratio of the different fragment ions reaching the electron multiplier. Quadrupole gain and offset are adjusted to set the proper peak width and mass axis for assigning masses. Finally, the electron multiplier voltage is set to compensate for the gain adjustment allowing the latter to remain at a consistent value. What is deemed a 'pass' versus 'fail' if defined at all, is not standardized. As such the process of tuning alone does not necessarily guarantee minimal variation across vendor platforms. The tuning dilemma is arguably exacerbated in a forensic setting because individual laboratories define standards of practice, including tuning frequency and instrument maintenance guidelines, and validate instruments under different operational settings.

The concern regarding comparability of mass spectra across vendor platforms is neither new nor unique to forensic applications. Decades ago, the Environmental Protection Agency (EPA) adopted the use of tune check compounds in order to address and reduce systematic variance within mass spectra; decafluorotriphenylphosphine (DFTPP) and bromofluorobenzene (BFB) were employed for semi-volatile and volatile analytes, respectively. In this approach, the instrument is tuned using the internal protocol and then a proscribed amount of a tune check compound is injected. The resulting spectrum, also generated by a proscribed processing procedure, is evaluated for specific $\mathrm{m} / \mathrm{z}$ values. Table $\mathbf{3 . 1}$ shows the most recent criteria published by the EPA for DFTPP. ${ }^{7}$ In this protocol, all DFTPP criteria must be met before case samples are analyzed. 
Table 3.1. EPA DFTPP Tuning Criteria.

\begin{tabular}{cc}
\hline $\boldsymbol{m} / z$ Value & Ion Abundance Criteria \\
\hline 68 & $<2 \%$ of 69 \\
69 & Present \\
70 & $<2 \%$ of 69 \\
127 & $40-60 \%$ of 198 \\
197 & $<2 \%$ of 198 \\
198 & Base peak or Present \\
199 & $5-9 \%$ of 198 \\
365 & $>1 \%$ of 198 \\
441 & Present, but $<150 \%$ of 443 \\
442 & Base Peak or Present \\
443 & $15-24 \%$ of 442 \\
\hline
\end{tabular}

Over the years, instrument vendors have added algorithms that adjust the tune of PFTBA to meet DFTPP criteria and processing algorithms that can check injected DFTPP for acceptance. It is important to note that the DFTPP criteria addresses the ability to detect isotopic peaks as well as abundance issues across a $\mathrm{m} / \mathrm{z}$ range that correlates well with most drugs of forensic interest. Accordingly, a study was designed to determine if this approach could be applied to seized drug analyses as a tool to minimize the variance of ion \% RAs across instrument platforms. Specifically, the hypothesis set forth was that when detector parameters from an autotune produced a DFTPP spectrum are deemed a pass, the variability of the spectrum obtained contemporaneously with this tune will vary less than those obtained following a tune that resulted in a DFTPP fail.

Somewhat surprisingly, the results showed that the DFTPP pass/fail outcome had no significant effect on the variability of \%RAs, with the exception of cathinones. However, the differences were minimal and not substantial enough to recommend its use in a forensic setting. The results did demonstrate that tuning frequency, rather than tuning to specific criteria, is the key factor influencing variation within and between autotunes. Specifically, in this study a tune- 
then-run scheme was performed, and the results showed that the \%RSD of \%RAs decreased fivefold compared to the previous study. Thus, it is recommended that forensic laboratories measure performance criteria, either from the autotune report or via a performance check that indicates unacceptable performance and to develop a schedule dictating when maintenance, ranging from column clipping to source cleaning, must be performed. Within these performance brackets, the autotuning process should be conducted immediately before each case sample batch to minimize variation in \%RA values across vendor platforms.

\subsection{Materials and Methods}

\subsubsection{Chemicals and Reagents}

LC-MS grade methanol was purchased from Fischer Scientific (Fair Lane, NJ). The DFTPP standard was obtained from Supelco Analytical (Bellefonte, PA) and all NPS standards were obtained from Cayman Chemical (Ann Arbor, MI). For each instrument, a total of three solutions were prepared: the check solution and two drug solutions each containing three NPSs, one cathinone and two cannabinoids. The NPSs were selected to include a range of molecular weights and spectral features; Table 3.2 summarizes the mixtures and compounds utilized and the designated compound feature class, defined in section 3.2.3.

Table 3.2. Composition of Check Mix and NPS drug mixes.

\begin{tabular}{ccccc}
\hline Solutions & Compounds & $\begin{array}{c}\text { Concentration } \\
(\mathbf{p p m})\end{array}$ & $\begin{array}{c}\text { Feature } \\
\text { Class }\end{array}$ & $\begin{array}{c}\text { Molecular Weight } \\
(\mathbf{g} / \mathbf{m o l})\end{array}$ \\
\hline Check Solution & DFTPP & 100 & -- & 442 \\
\hline \multirow{3}{*}{ NPS Solution 1 } & NEB ${ }^{* a}$ & 150 & Poor & 191 \\
& JWH-116 & 200 & Rich & 369 \\
& JWH-200 & 250 & Poor & 384 \\
\hline \multirow{2}{*}{ NPS Solution 2 } & MeMABP*b & 150 & Poor & 191 \\
& JWH-149 & 200 & Rich & 369 \\
& BB-22 & 300 & Poor & 384 \\
\hline
\end{tabular}

*Cathinone, ${ }^{\mathrm{a}} \mathrm{N}$-Ethylbuphedrone, ${ }^{\mathrm{b}}$ 4-Methybuphedrone. IUPAC naming for all six compounds are shown in the abbreviations. 


\subsubsection{GCMS Instrumentation}

Prior to the start of the project, routine maintenance including source cleaning, replacing the injection syringe, column clipping, changing inlet liner, and changing the injection port septum was performed on both instruments, an Agilent (A) and a Perkin Elmer (B). The routine maintenance was performed as needed, i.e. manufacturer recommendations and/or instrumental indicators, throughout the duration of the study. The additional maintenance of sanding and cleaning the capillary inlet gold seal was performed on instrument B between days 6 and 7. This maintenance was performed due to a low inlet pressure warning along with significant changes in the retention time of DFTPP. These instrumental indicators are typically associated with the inlet liner and septum needing to be changed, however, this did not remedy the reduced pressure warning. Upon investigation, the gold seal was noticeably worn where contact occurs between the injector nut and capillary injector fitting. Resurfacing and cleaning resulted in the GC inlet properly maintaining pressure.

The software used for both instruments include multiple autotune options including a 'basic' autotune and an autotune which attempts to adjust tune settings so that a subsequent analysis of DFTPP would meet EPA criteria. Instrument A provides three separate autotune options ('basic', DFTPP, and BFB), the 'basic' autotune function was used. Instrument B provides two tuning options one labeled as "Custom (Autotune)" and one labeled as "Standard (DFTPP/BFB)" the latter was the tune method used for this study. Because instrument B does not have separate BFB and DFTPP options, it was assumed that the algorithm adjusts settings to meet both criteria. Instrument details and method settings are shown in Table 3.3. 
Table 3.3. GC-MS systems details, method/instrument settings, and instrument/analysis software.

\begin{tabular}{|c|c|c|c|}
\hline & Setting & A & B \\
\hline \multirow{8}{*}{ GC } & Model & 7890B & Clarus 680 \\
\hline & Column Type & HP-5 & ZB-5MS \\
\hline & Column L X ID X df (m X mm X $\mu \mathrm{m})$ & $30 \times 0.25 \times 0.25$ & $20 \times 0.18 \times 0.18$ \\
\hline & Injection Split Ratio & $20: 1$ & $10: 1$ \\
\hline & Injection Temperature $\left({ }^{\circ} \mathrm{C}\right)$ & 250 & 250 \\
\hline & Initial Column Temperature $\left({ }^{\circ} \mathrm{C}\right)$ & 100 & 100 \\
\hline & Final Column Temperature $\left({ }^{\circ} \mathrm{C}\right)$ & 320 & 315 \\
\hline & Ramp & 20 & 20 \\
\hline \multirow{4}{*}{ MS } & Model & 5977AMSD & Clarus SQ 85 \\
\hline & Source Temperature $\left({ }^{\circ} \mathrm{C}\right)$ & 250 & 250 \\
\hline & EM Voltage & $2504-2864$ & $1685-1874$ \\
\hline & Scan Range & $\begin{array}{l}\text { DFTPP Method: } 40-500 \\
\text { NPS Method: } 40-400\end{array}$ & $40-500^{*}$ \\
\hline \multirow{4}{*}{ Software } & Instrument Control & MassHunter GC/MS Acquisition & Turbo Mass \\
\hline & Version & B.07.00 SP1.1549 & 6. 1.0 .1983 \\
\hline & Data Analysis & Qualitative Analysis & Turbo Mass \\
\hline & Version & B.06.00 & 6.1.0.1983 \\
\hline
\end{tabular}

${ }^{*}$ The day 1 scanning range was 40-400. 


\subsubsection{Data Acquisition and Processing}

The process used to collect, screen, combine, and collate mass spectra data is summarized in Figure 3.1. Each instrument was tuned immediately prior to starting the day's 'analytical batches'. An analytical batch consisted of blanks, the check solution, and each NPS solution. A data set was defined as a single repetition of the check solution and ten (10) repetitions of each NPS solution (and all necessary blanks) within a single day. DFTPP tuning criteria was not checked at the time of analysis since it was not used as a criterion for deciding if analysis should continue. Analytical batches were repeated using this same process over 2 and 1.5 weeks for instruments A and B, respectively. Ten data sets for each instrument were collected over this time yielding a total of 100 mass spectra or analytical batches from each instrument for each NPS.

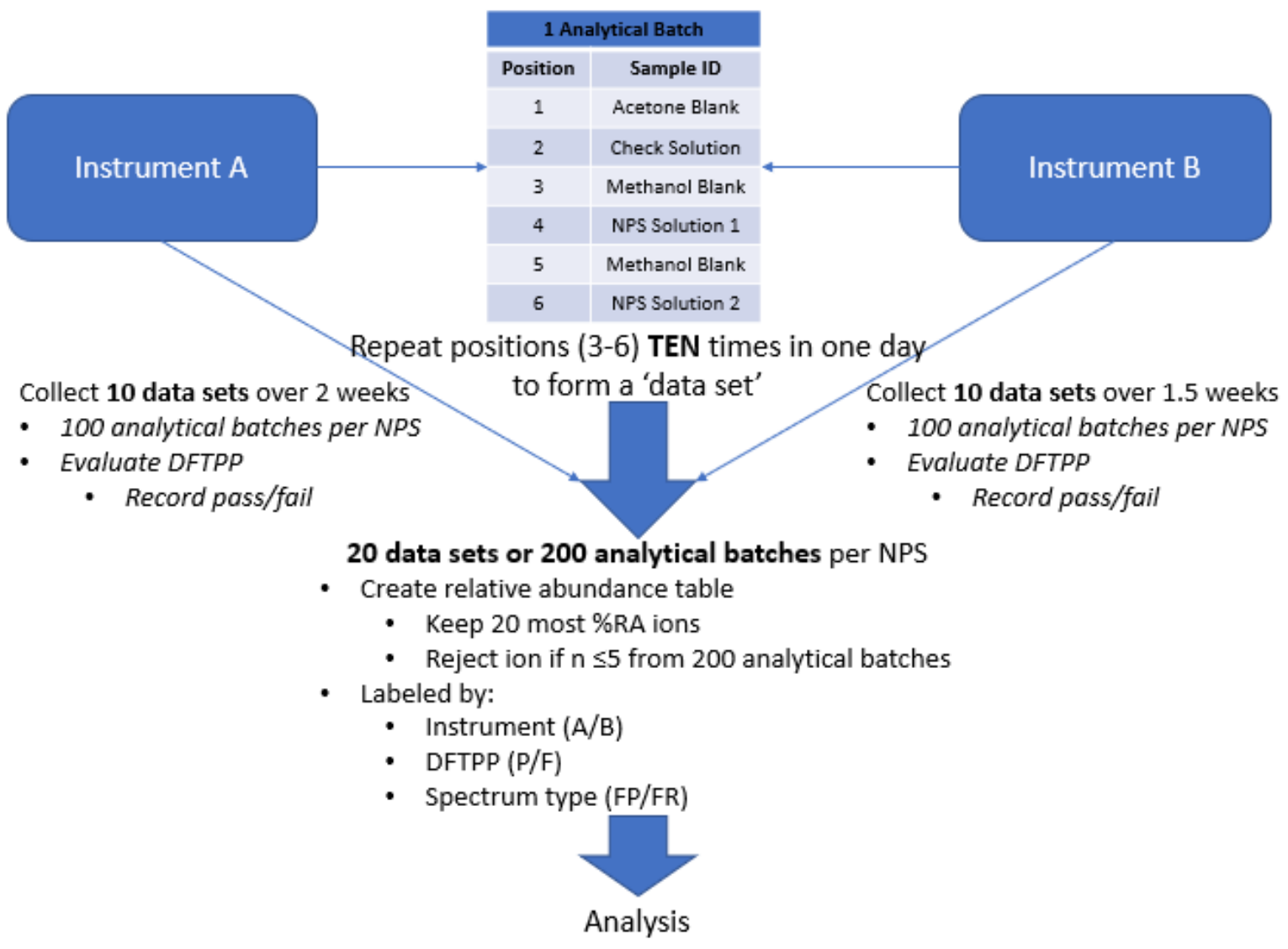

Figure 3.1. Collection and collation of data procedure. 
The mass spectra were obtained from the average across the chromatographic peak at the estimated FWHM followed by subtraction of the averaged background obtained prior to and after elution. The \%RA of DFTPP criteria ions were calculated and were compared against the EPA criteria (Table 3.1) to determine whether the tune passed or failed. If all criteria ion RAs were within the established range, the tune was deemed a pass, oppositely, a tune was deemed a fail if a single ion \%RA was outside of the criterion range. For each NPS, the \%RA of the 20 most abundant $\mathrm{m} / \mathrm{z}$ values were recorded. The choice of 20 ions was selected based on prior work in our laboratory. ${ }^{6}$ Data was verified two additional times by at least two individuals after initial entry, once during data processing and again prior to statistical analysis. Spectra were categorized as feature-rich and feature-poor, a subjective description term defined during previous work. ${ }^{6}$ An example illustrating the difference is presented in Figure 3.2. Feature-poor compounds produce mass spectra with a limited number of fragment ions often in low abundance, while feature-rich compounds fragment extensively and ions have varying abundances.

Once data collection was complete and collated for each compound, an initial data elimination was performed. Data was not eliminated based on an arbitrary \%RA value or estimated S/N considerations, rather, a $\mathrm{m} / \mathrm{z}$ value for a given compound was required to be present in more than $2.5 \%$ of the data sets to be included for further consideration. In other words, if a given ion from a compound was present in less than 5 spectra of the 200 collected, that $m / \mathrm{z}$ value was eliminated. Data analysis was then conducted using OriginLab® (OriginLab Corporation, Northampton, MA). 

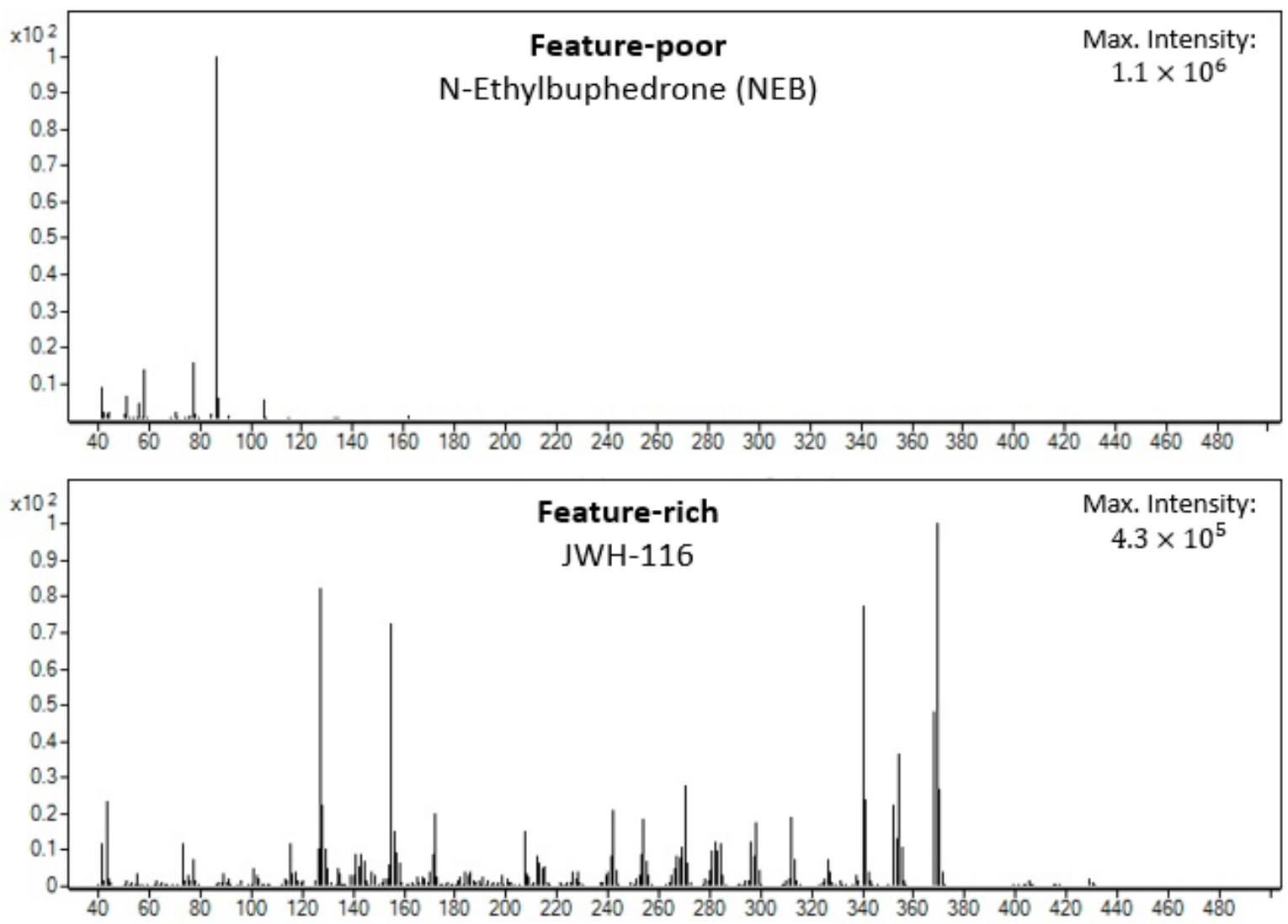

Figure 3.2. Comparison of spectra classified as feature-rich and feature-poor.

The $\mathrm{x}$-axis is \%RA and $\mathrm{y}$-axis is $m / z$ value. Both spectra originated from instrument A, day 4 , analytical batch 2. Maximum signal intensity is shown to demonstrate spectra are not result of weak detector signal.

\subsection{Results and Discussion}

The data was evaluated to address three questions:

1. Does the use of DFTPP as a tuning check reduce variation of \%RA values?

2. How does tuning frequency impact this variation?

3. Does the complexity of a spectrum (expressed as feature-rich/feature-poor) influence the variation of \%RA under these conditions?

\subsubsection{Tuning to DFTPP Criteria}

\subsection{1a Frequency of Pass/Fail}


Instrument A passed $100 \%$ of the DFTPP tune checks while instrument B passed only 20\% (Day 4 and 10). The most frequently failed ion was $m / z, 68$ followed by $m / z, 127 ; m / z, 199$ failed to meet criterion on a single day. No ions above $\mathrm{m} / \mathrm{z} 200$ failed to meet EPA criteria. $\mathrm{m} / \mathrm{z}$ 199 was the only ion to fail to meet the criterion due to being under \%RA threshold, this was by $\sim 0.5 \%$ RA. All other ions failed criteria due to being above the \%RA criterion threshold. The largest and smallest fail was $3.1 \%$ and $<0.1 \%$ above the $\%$ RA threshold, respectively. As previously mentioned the "Standard (DFTPP/BFB)" tuning option was used for instrument B. BFB is the lower molecular weight tune check compound utilized in EPA purge-and-trap methods for volatile organics and all criteria ions for this compound are less than $m / z$. 200. Thus, the tuning algorithm may emphasize low mass ions which would explain the DFTPP ions failing criteria due to low mass ion \%RAs being over the threshold. This supports the assumption that the tuning algorithm adjusts settings to attempt to meet both DFTPP and BFB criteria.

\subsection{1b Effect of Pass/Fail on Variability}

If passing the DFTPP criteria reduced ion \%RA variation, then data associated with passing tunes should demonstrate less variability than data collected on days when the tune criteria was not satisfied. Since failing tunes were only noted with Instrument B this was the only data considered. Results are summarized in Figures 3.3a-c. 


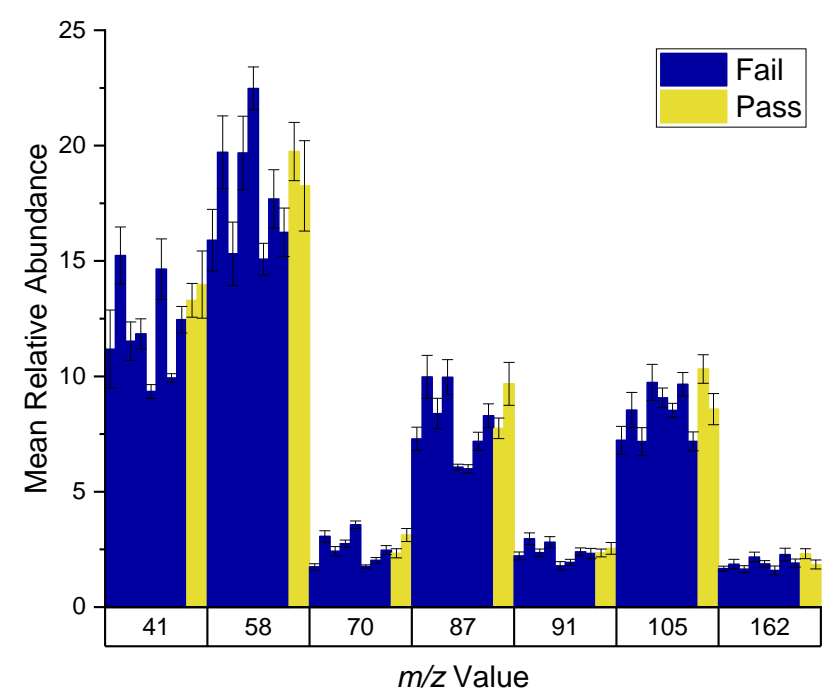

Figure 3.3a. Mean \% relative abundance per day for select ions from NEB separated by DFTPP tune pass and fail days.

Only instrument B was considered $(n=10)$. Note the $y$-axis scale change. Error bars indicate one standard deviation.

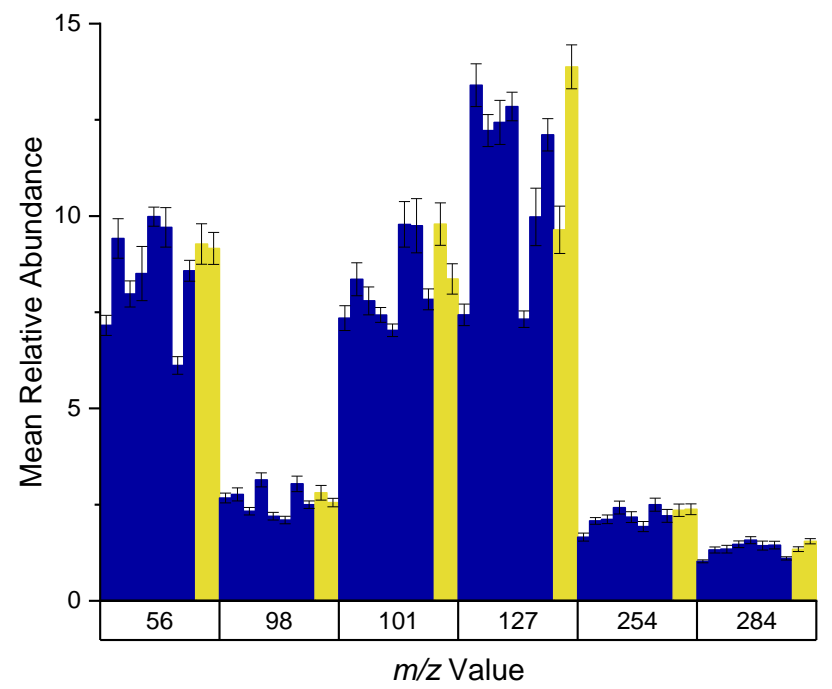

Figure 3.3b. Mean \% relative abundance per day for select ions from JWH-200 separated by DFTPP tune pass and fail days.

Only instrument B was considered $(n=10)$. Note the $y$-axis scale change. Error bars indicate one standard deviation. 


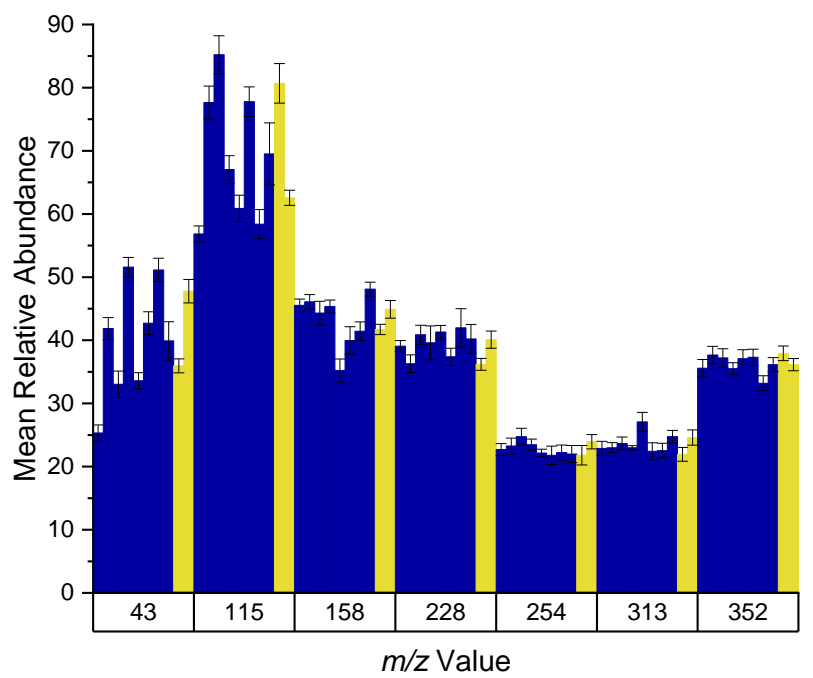

Figure 3.3c. Mean \% relative abundance per day for select ions from JWH-149 separated by DFTPP tune pass and fail days.

Only instrument B was considered $(n=10)$. Note the $y$-axis scale change. Error bars indicate one standard deviation.

Some ions show a significant between-day variability such as NEB $m / z 41,58$, and 87; JWH-200 m/z 127; and JWH-149 m/z 115; these ions also had increased within-day standard deviations relative to the ions showing consistent between-day mean \%RAs. Both patterns held regardless of DFTPP pass/fail criteria. This suggests that patterns of within-day variation follow patterns of between-day variation and that the DFTPP pass/fail criteria is not significant contributing factor. A second, but similar, observation derived from Figure 3.3 is that there are several $m / z$ values that show little inter- and intra-day variability such as $m / z 91$ and 162 from NEB; $m / z$ 98, 254, and 284 from JWH-200; and $m / z$ 254, 313, and 352 from JWH-149. These patterns were also consistent regardless of DFTPP performance which further suggests DFTPP criteria outcome is not a significant factor in variation reduction. This also suggests that other underlying factors contribute to the degree of variability observed.

\subsection{1c t-test of Means and f-test of Variance Tune Pass/Fail}


A t-test of means and a f-test of variance was performed comparing the pass/fail groupings $(\alpha=0.05)$. The results are summarized in Figure 3.4. Two feature-poor spectra (NEB and MeMABP) had the most statistically significant differences between mean relative abundances for passing versus failing the DFTPP tuning criteria. However, this pattern does not hold for the cannabinoids. The results for both feature-poor and -rich cannabinoids is split, where one compound consistently shows statistically significant differences in mean and variance, but the other compound from the same feature class does not. This is further evidence that the pass/fail criteria is not a significant factor in \%RA variability and the implementation of DFTPP tune checks within a forensic setting would be of little value.

\begin{tabular}{|c|c|c|c|c|c|c|c|c|c|c|c|c|}
\hline \multirow{2}{*}{ Comparison } & \multicolumn{2}{|c|}{ NEB ${ }^{*}$} & \multicolumn{2}{|c|}{ MeMABP* } & \multicolumn{2}{|c|}{ BB-22 } & \multicolumn{2}{|c|}{ JWH-200 } & \multicolumn{2}{|c|}{ JWH-116 } & \multicolumn{2}{|c|}{ JWH-149 } \\
\hline & $\operatorname{lon}(\mathrm{m} / \mathrm{z})$ & Pass-Fail & Ion $(\mathrm{m} / \mathrm{z})$ & Pass-Fail & Ion $(\mathrm{m} / \mathrm{z})$ & Pass-Fail & Ion $(\mathrm{m} / \mathrm{z})$ & Pass-Fail & Ion $(\mathrm{m} / \mathrm{z})$ & Pass-Fail & $\operatorname{Ion}(\mathrm{m} / \mathrm{z})$ & Pass-Fail \\
\hline \multirow{5}{*}{ t-test of means } & 77 & & 91 & & 144 & & 127 & & 127 & & 354 & \\
\hline & 58 & & 57 & & 241 & & 56 & & 155 & & 369 & \\
\hline & 41 & & 65 & & 55 & & 101 & & 369 & & 169 & \\
\hline & 51 & & 42 & & 116 & & 155 & & 340 & & 141 & \\
\hline & 105 & & 44 & & 89 & & 70 & & 368 & & 368 & \\
\hline \multirow{5}{*}{ f-test of variance } & 77 & & 91 & & 144 & & 127 & & 127 & & 354 & \\
\hline & 58 & & 57 & & 241 & & 56 & & 155 & & 369 & \\
\hline & 41 & & 65 & & 55 & & 101 & & 369 & & 169 & \\
\hline & 51 & & 42 & & 116 & & 155 & & 340 & & 141 & \\
\hline & 105 & & 44 & & 89 & & 70 & & 368 & & 368 & \\
\hline
\end{tabular}

Figure 3.4. Comparison of performance as a function of tune pass/fail.

*: Cathinone. BB-22 and JWH-200 are feature-poor cannabinoids, JWH-116 and JWH-149 are feature-rich cannabinoids. Ions shown are five most abundant ions in decreasing order from instrument B. For the f-test the null hypothesis was considered to be that the variances from the two groups were equal.

\subsubsection{Relative Abundance}

\subsection{2a Within-Day Variation}

Previous work in our lab showed that \%RSD tends to increase exponentially as mean \%RA decreases. ${ }^{6}$ A similar pattern was observed in this study (Figure B1). The highest recorded 
$\%$ RSD in the present study was $\sim 49 \%$, a 5 -fold reduction compared to the initial study. ${ }^{6}$ Given that the DFTPP pass/fail criteria did not have a significant impact on variability, the remaining factor is tune frequency. Thus, assuming a detector is operating within a defined range of acceptable performance, tuning as close to analysis time as possible is the key factor in minimizing variance in \%RA.

To determine if one compound classification produces more variation than another, the plot described above was separated by the feature class designations (Figure 3.5). Feature-poor cannabinoids produced the most data points with $\geq 15 \%$ RSD, 23 ions, followed by feature-rich cannabinoids, 6 ions, cathinones produced the least with only 4 ions. Regardless of mass spectral richness, synthetic cannabinoids have comparatively more complex structures than cathinones, which suggests that structural complexity may contribute to \%RA variation. However, the current data does not provide the information necessary to determine the exact source(s) of the observed differences. 


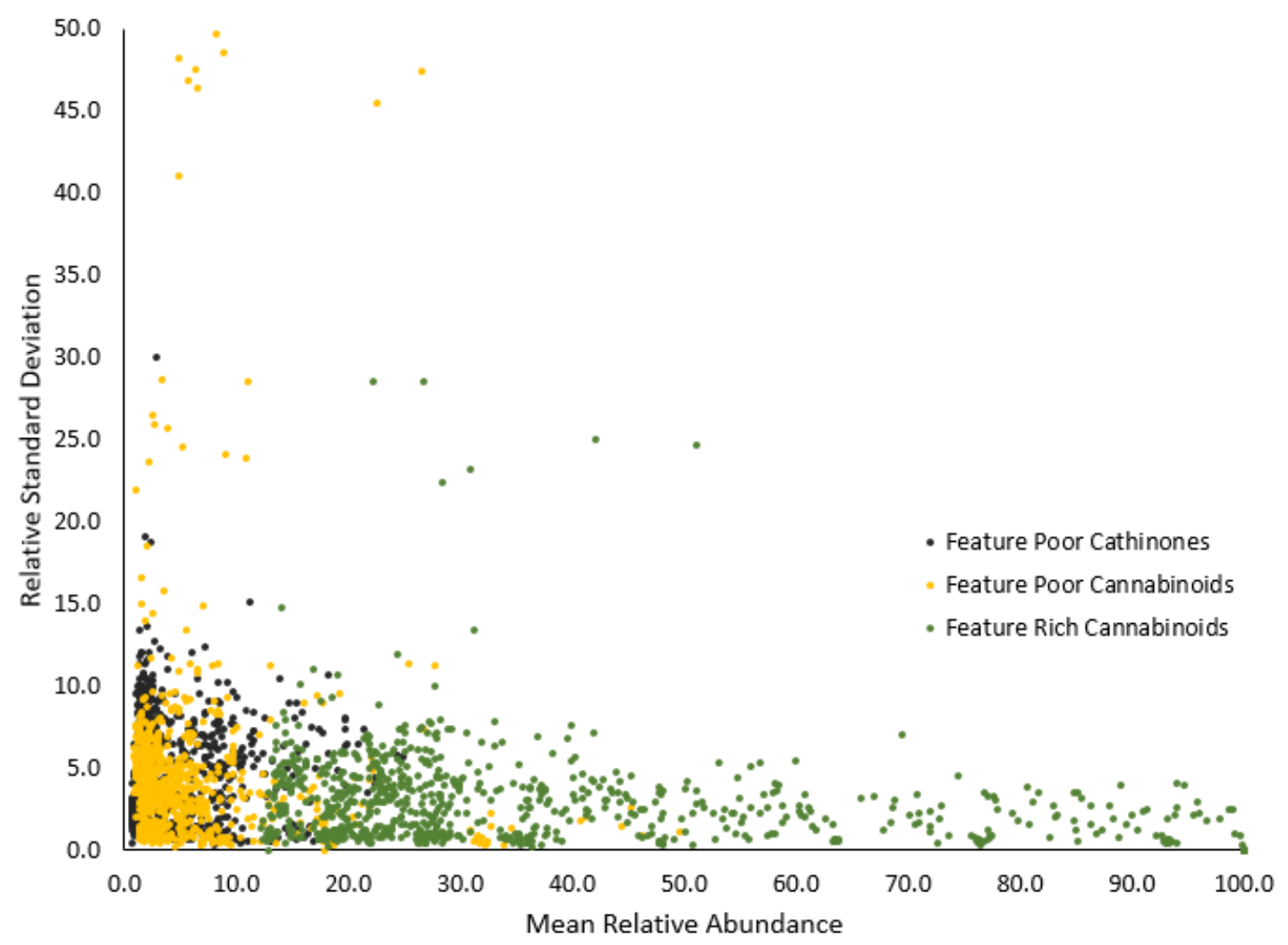

Figure 3.5. Relative standard deviation plotted as a function of the mean relative abundance from single day analysis sets $(\mathrm{n}=10)$ from all NPSs and separated by NPS category and subclass.

The current data indicates that consistent tuning, within-day variation generally lies $\leq 15 \%$ RSD but, is reliably within $50 \%$ RSD. This poses unique challenges for NPS identification, particularly in a forensic setting, as isomeric structures can produce near identical spectra. In a scenario where a non-targeted analyte identification is dependent on the \%RA of a handful of ions, accounting for a 50\% RSD within each individual \%RA poses a challenge. In a more complex scenario, two structural isomers may have different scheduling classifications but nearly identical spectra. Identification of the specific structural isomer determines whether a suspect is in possession of an illegal substance or an unregulated, legal substance; here, accounting for the mass spectral variation is even more crucial. In either scenario, other information is relied upon or additional analyses are performed to make identifications, for instance GC retention times/indices and DART-TOF, respectively. However, the large \%RSD 
reported here highlights the importance of utilizing all information or performing multiple analyses in complex, non-targeted isomer identifications.

\subsection{2b Between-Day Variation}

An important goal of this study was to investigate data trends and variation from both within a single autotune as well as between different autotunes. Initial examination of the latter was performed by creating group plots of all recorded \%RAs for individual ions (Figures B2ac). The same trend described in section 3.3.1b is seen, where some ions show consistent \% RAs over the course of the study while others vary drastically. In theory, a single autotune should produce nearly identical mass spectra for a single compound. Under this premise, the \% RA means from individual days were calculated for ions and formatted into similar group plots (Figures 3.6a-c).

Although the same trend continues, where some ions have comparably more consistent $\%$ RAs than other ions, instrument A produces less between-day, and therefore between tune, variation than instrument $B$. As described above, instrument B tune checks failed mainly due to criteria ions below $m / z 200$ having \%RAs over the threshold. Synthetic cathinones in this study only produced ions below $\mathrm{m} / \mathrm{z} 200$ (Figures 3.6a and B3a) whereas synthetic cannabinoids (Figures 3.6b, 3.6c, B3b, and B3c) produced ions above and below $m / z$ 200. Specifically looking at instrument B, ions above $m / z 200$ show more between-day \%RA consistency than ions below the threshold. Because the $\mathrm{m} / \mathrm{z}$ threshold value is identical for both tune check fails and between-day consistency differences, it is hypothesized that the between-day inconsistency of low mass ions is largely due to tune biasing. Here, ions below the $m / z 200$ threshold have an innate tuning bias to meet the EPA criteria for BFB as a tune check compound. Because the autotune parameters are more variable below this threshold, due to the tuning algorithm 
attempting to meet both DFTPP and BFB criteria, the low mass mean \%RAs have a higher variability between days.

It could be argued that this trend is observed on instrument A for JWH-149 (Figure 3.6c) because of the variability differences above and below $\mathrm{m} / \mathrm{z} 200$, for example comparing $\mathrm{m} / \mathrm{z} 115$ and $m / z$ 352. However, the ion \%RAs of NEB and JWH-200 are more consistent between days (Figures 3.6a and 3.6b) suggesting that this is not the case. In other words, these ions show consistent between-day means regardless of the $\mathrm{m} / \mathrm{z}$ value and therefore the variability trend describe for instrument $\mathrm{B}$ is not present on instrument $\mathrm{A}$. It is believed this trend is specific to instrument B and a result of the tuning bias toward lower masses.

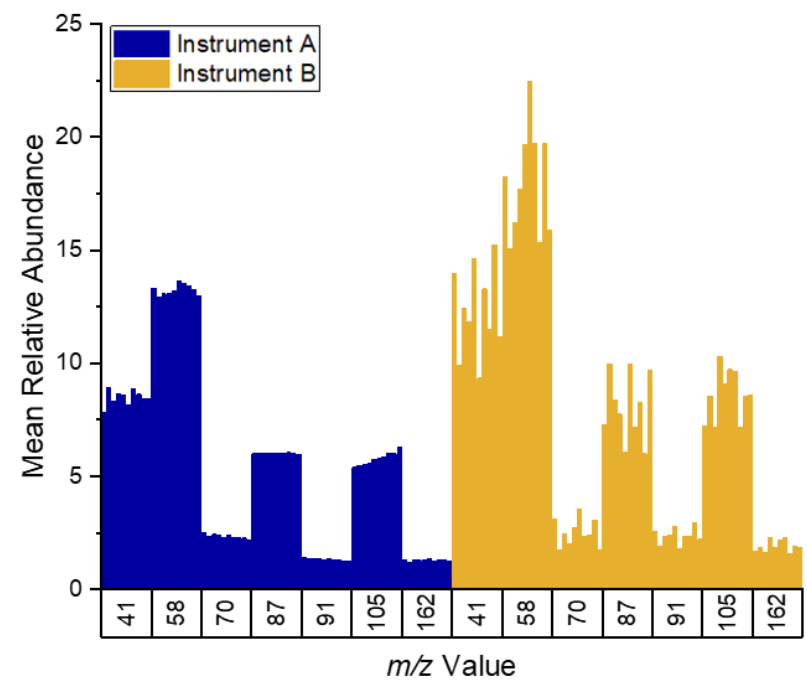

Figure 3.6a. Mean RA per day for select ions from NEB. $\mathrm{n}=3$ to 10 depending how many times the ion was recorded each day. Note $\mathrm{y}$-axis scale change. 


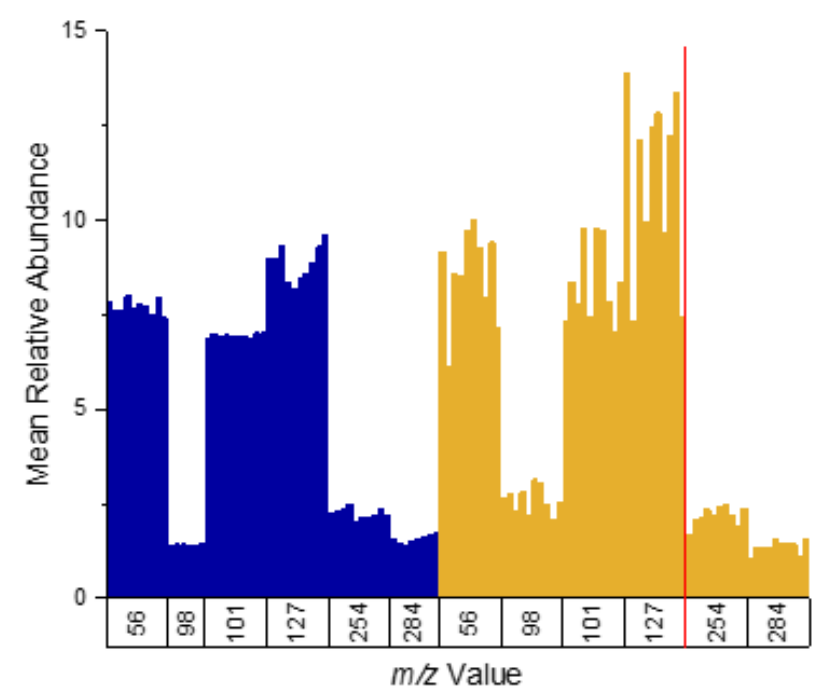

Figure 3.6b. Mean RA per day for select ions from JWH-200.

$\mathrm{n}=3$ to 10 depending how many times the ion was recorded each day. Red lines indicate threshold of $\mathrm{m} / z, 200$ where ion variation from instrument B comparatively decreases. Note yaxis scale change.

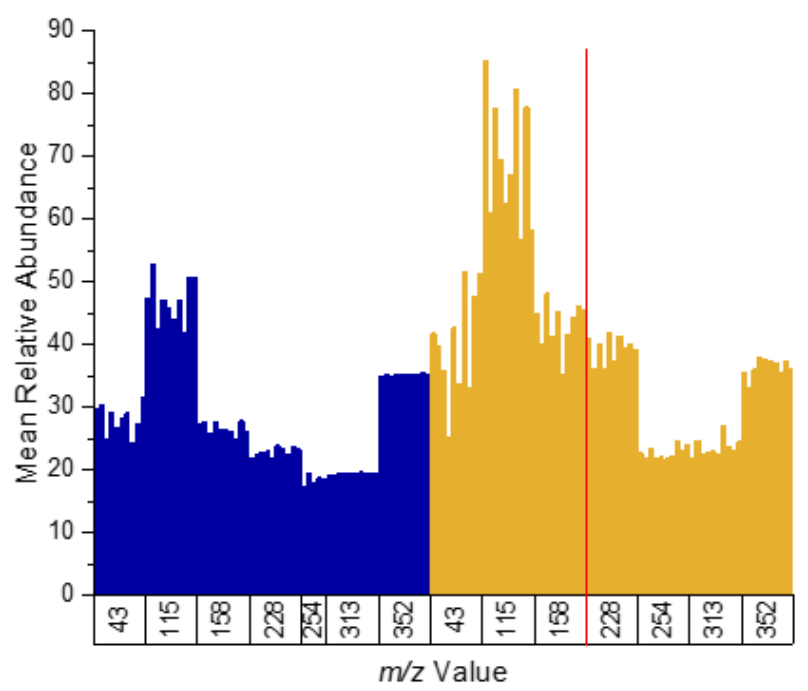

Figure 3.6c. Mean RA per day for select ions from JWH-149.

$\mathrm{n}=3$ to 10 depending how many times the ion was recorded each day. Red lines indicate threshold of $\mathrm{m} / \mathrm{z} 200$ where ion variation from instrument B comparatively decreases. Note yaxis scale change.

\subsection{2c Ion Variation and Fragmentation Correlation}

As a final note, an underlying trend was consistently present within the data. Some ions showed consistent within- and between-day \%RAs as measured by single day standard deviations and consistency of mean \%RAs, respectively. Other ions showed variable within- and 
between-day \%RAs. This was initially postulated to be the result of differences in fragmentation patterns and/or the ability to fragment via multiple pathways. Two scenarios were hypothesized to contribute to the variation differences. Firstly, if an ion was formed through multiple fragmentation pathways then the variation would be higher and conversely if an ion could only be formed by a single pathway then the variation would be smaller. Secondly, if an ion was unlikely to be formed during a fragmentation event then the variation would be larger; for example, in a fragmentation event where one ion is statistically more likely to carry the charge, then the other ion fragment would be formed inconsistently and therefore have a higher variation. These hypotheses would also address the observation that the same $\mathrm{m} / \mathrm{z}$ values from different compounds produced different amounts of variation. Both observations, which link fragmentation patterns/ability and variation differences, are similar to data reported by Jackson et. al. ${ }^{8}$ The authors reported that the uncertainty of fragment ions pairs were correlated, in other words, \%RA uncertainty is not independent of fragmentation. ${ }^{8}$ To address this, the identity of fragment ion structures, corresponding to both variable and consistent $\mathrm{m} / \mathrm{z}$ values, were found in the NIST MS Interpreter 2.0 (version 1.6/1.7/2.0). This hypothesis was confirmed with several ions, in which a variable $\mathrm{m} / \mathrm{z}$ value corresponded to an ion which could be formed through multiple possible pathways but failed for other $\mathrm{m} / \mathrm{z}$ values. The study presented here was not designed to address the link between fragmentation pathways and ion variation and it is beyond the scope of the author's expertise. It is the author's opinion that this phenomenon is worthy of investigation and a study should be designed to address the correlation of ion variation and fragmentation patterns/ability.

\subsection{Conclusions}


Employing an independent tune check using DFTPP was not effective in reducing the variability observed in relative abundance values within- and between-days. Rather tuning frequency and tuning just prior to analysis was critical in minimizing this variation. Additionally, slight differences in tuning algorithms between vendors was noted and should be considered when comparing mass spectra. If an instrument is shown to be operating within laboratory defined acceptability criteria, frequent tuning is the best practice. When followed, regular tuning reduces the $\%$ RSD of relative abundances 5 -fold compared to infrequent tuning. However, laboratories should appreciate that the intrinsic variation can be as high as 50\% RSD, a factor that must be considered when structural identifications are made.

\subsection{Funding and Acknowledgements}

This work was supported by the United States Department of Commerce, National Institute of Standards and Technology Award \# 70NANB16H105. The authors wish to acknowledge the efforts of undergraduate researcher Benjamin Alderson who collated and double-checked data.

\subsection{References}

1. Temerdashev, A.; Grigor'ev, I.; Rybal'chenko, I., Evolution of new narcotic substances and methods of their determination. Journal of analytical chemistry 2014, 69 (9), 817-844.

2. Archer, R. P.; Treble, R.; Williams, K., Reference materials for new psychoactive substances. Drug Test. Anal. 2011, 3 (7-8), 505-514.

3. Namera, A.; Kawamura, M.; Nakamoto, A.; Saito, T.; Nagao, M., Comprehensive review of the detection methods for synthetic cannabinoids and cathinones. Forensic toxicology 2015, 33 (2), 175-194. 
4. Zuba, D., Identification of cathinones and other active components of 'legal highs' by mass spectrometric methods. TrAC Trends in Analytical Chemistry 2012, 32, 15-30.

5. Elie, M. P.; Elie, L. E.; Baron, M. G., Keeping pace with NPS releases: fast GC-MS screening of legal high products. Drug testing and analysis 2013, 5 (5), 281-290.

6. Kelly, K.; Bell, S., Evaluation of the reproducibility and repeatability of GCMS retention indices and mass spectra of novel psychoactive substances. Forensic Chemistry 2018, 7, 10-18. 7. GAS, S. O. C. B., METHOD 8270E SEMIVOLATILE ORGANIC COMPOUNDS BY GAS CHROMATOGRAPHY/MASS SPECTROMETRY. 2017.

8. Davidson, J. T.; Lum, B. J.; Nano, G.; Jackson, G. P., Comparison of measured and recommended acceptance criteria for the analysis of seized drugs using Gas ChromatographyMass Spectrometry (GC-MS). Forensic Chemistry 2018, 10, 15-26. 


\section{Chapter 4: Synthetic Small Molecule Characterization and Isomer Discrimination Using Gas-phase Hydrogen Deuterium Exchange}

Kelly, K.; Maleki, H.; Valentine, S.; Bell, S. Submitted for review to Analytical Chemistry.
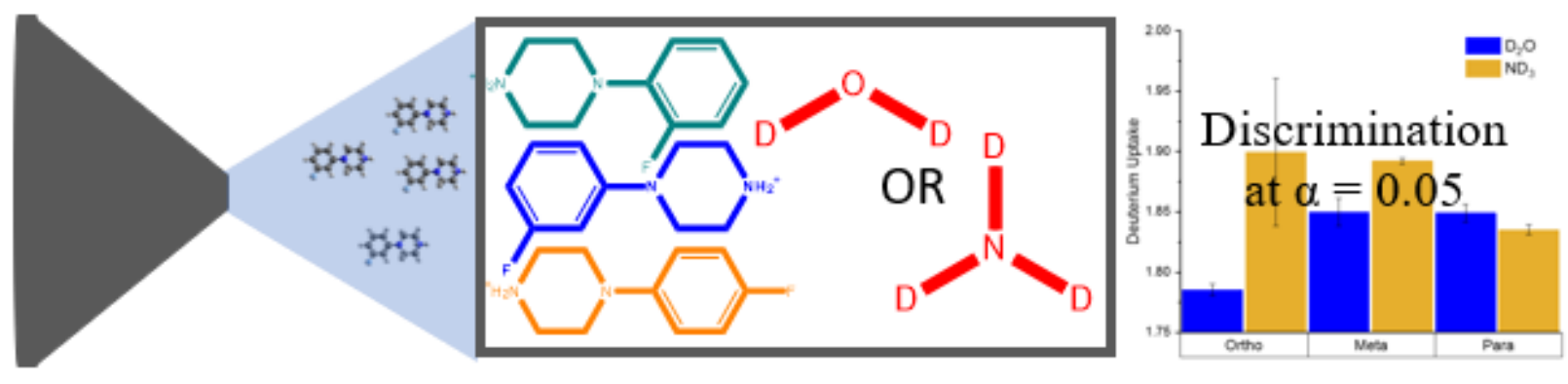

\subsection{Introduction}

Novel psychoactive Substances (NPSs) describes various classes of small synthetic compounds designed to mimic the effects of specific illicit drugs. Although some analogues are currently regulated by agencies worldwide, slight side chain substitutions allow new analogs to evade regulations. ${ }^{1-2}$ The 1986 Anti-Drug Abuse Act in the United States was created to extend the drug legislation to analogues sold for human consumption. ${ }^{3}$ NPSs are commonly sold using a variety of marketing strategies to circumvent this amendment. ${ }^{1-2,4-5}$ This combination of strategies has created an endless cycle where a new analog is readily available as a replacement once an existing analog is scheduled. ${ }^{4}$ The constant influx of new compounds has forced forensic laboratories to determine which NPSs are new analogs and which are already characterized.

Various detection methods have been employed for the analysis of NPSs in both seized materials and biological samples. ${ }^{1,5}$ The current analytical trend is to utilize high-resolution mass spectrometry (HRMS) for proposed identity confirmation, by means of accurate mass, as well as structural elucidation by MS/MS. ${ }^{2}$ However, there are several drawbacks with this approach. First, this method is not capable of identifying regio-isomers. Secondly, interfaced chromatographic separation methods are often time consuming and do not provide unambiguous 
identifications. One technique showing promise to overcome some of these drawbacks is ion mobility spectrometry (IMS) coupled to MS.$^{6-11}$ IMS is currently employed as a presumptive screening test for illicit substances in high traffic locations, (i.e., mail depots, airports, border crossings, etc.). ${ }^{10}$ Despite its employment as a non-confirmatory technique in forensic applications, IMS-MS instrumentation is continually being developed and modified. These advancements have increased resolving power, improved the detection limits for components in complex mixtures, and helped improve structural inferences. ${ }^{6-7,9,12}$ Due to the complexity of isomer identification and the increased development of IMS and similar instrumentation, a paradigm shift is occurring where high-speed measurements related to different physiochemical property of compounds are bearing fruit. ${ }^{13-21}$

Solution-phase hydrogen deuterium exchange (HDX) has a rich history in the study of protein structure using MS techniques ${ }^{22-23}$ and is increasingly applied in the development of pharmaceuticals ${ }^{24-26}$; its usage for aiding in the identification of complex mixture components is relatively limited. Over the years, several have proposed using the mass-to-charge $(\mathrm{m} / \mathrm{z})$ shift of metabolites exposed to $\mathrm{D}_{2} \mathrm{O}$ as an indicator of the number of acidic hydrogens..$^{27-29}$ This measurement is used as a supplement to the measured precursor ion $\mathrm{m} / \mathrm{z}$ values and chromatographic retentions times in order to reveal the identities of mixture components. More recently, gas-phase HDX has been used for biomolecule ion structure elucidation ${ }^{30-35}$ as well as to examine its potential for distinguishing metabolites in complex mixtures. ${ }^{36-38}$ Overall the gas phase approach offers advantages in measurement speed and reproducibility as well as offering significant potential for predicting the HDX behavior of compounds for which data have not been previously recorded. Notably, experiments have been performed for compounds containing a limited number of exchangeable hydrogens. ${ }^{37}$ More recently, compositional and linkage 
isomers of oligosaccharides were distinguished using HDX despite these isomers not being resolved using IMS alone. ${ }^{38}$ To date, gas-phase HDX has not been applied to aid in the identification of NPSs.

This proof-of-concept study utilized gas-phase HDX-IMS-MS for the analysis of select NPSs (compound names and structures shown in Table C1) in the presence of two different reagent gases, $\mathrm{D}_{2} \mathrm{O}$ and $\mathrm{ND}_{3}$. Of the six initial NPSs analyzed for exchange propensity, one compound showed no exchange in the presence of either reagent gas. Four of the six NPSs showed efficient exchange in the presence of $\mathrm{ND}_{3}$ but little to no exchange in the presence of $\mathrm{D}_{2} \mathrm{O}$. The only compound which showed exchange with both reagent gases was benzyl piperazine; it was hypothesized that the six-membered diamine ring facilitated exchange in the presence of $\mathrm{D}_{2} \mathrm{O}$. Three disubstituted aromatic ring isomers (ortho-, meta-, and parafluorophenyl piperazine), in which one substituent is a diamine ring, were then analyzed to determine if gas-phase HDX propensity could discriminate among the fluorophenyl piperazine isomers. It was shown that complete discrimination, with the current instrumentation, was possible at the $\alpha=0.05$ level. Importantly, deuterium exchange of both reagent gases is required for complete isomer discrimination between ortho-, meta-, para-fluorophenyl piperazine. Molecular dynamics (MD) simulations provide insight into the underlying molecular behavior which is hypothesized to contribute to the deuterium exchange differences.

\subsection{Materials and Methods}

\subsubsection{Chemicals and Reagents}

Deuterated ammonia $\left(\mathrm{ND}_{3}\right)$ and water $\left(\mathrm{D}_{2} \mathrm{O}\right)$ was purchased from Sigma-Aldrich(St. Louis MO). LCMS grade methanol and Optima LCMS grade acetonitrile were obtained from Fisher Scientific (Fair Lawn NJ). Glacial acetic acid was obtained from EM Science (Gibbstown 
NJ). NPS standards were purchased from Cerilliant (Round Rock TX) and Cayman Chemical (Ann Arbor MI). The six initial NPSs studied were prepared in standard solutions at concentrations of $10 \mathrm{ppm}$ in 1:1 methanol:acetonitrile and 1\% acetic acid. The three fluorophenyl piperazine isomer standards were at concentrations of $50 \mathrm{ppm}$ in the same solvent proportions. Details regarding the concentration change are described in Section 4.2.3.

\subsubsection{Experimental Design}

The six initial NPSs were chosen based on potentially labile hydrogen atoms, specifically amine functional groups (Table 4.1). The fluorophenyl piperazine isomers were chosen because they contained a diamine ring, which was linked to the propensity of HDX in the presence of $\mathrm{D}_{2} \mathrm{O}$. It is instructive to note that isomeric compounds selected for this study have been analyzed by GCMS. In these experiments, the retention times were nearly identical indicating the compounds could not be resolved by GC.

\subsubsection{Instrumentation}

The instrument used in this study has been described in detail previously. ${ }^{13,36} \mathrm{~A}$ brief description of instrumentation is presented here, and an instrument schematic is provided as Figure C1. The instrument consists of a home-built drift tube coupled with an LTQ Velos linear ion trap (ThermoScientific, San Jose, CA, USA) mass spectrometer. Samples are infused at a rate of $300 \mathrm{~nL} / \mathrm{min}$ via a pulled-tip capillary biased at $+2000 \mathrm{~V}$ relative to the entrance of a differentially-pumped desolvation region. The ions are focused in an hour-glass ion funnel ${ }^{13}$ and temporarily stored in the first ion gate (G1) all contained within the desolvation region. The ions are periodically pulsed $(\sim 50 \mathrm{~Hz})$ into the drift tube, which is filled with He buffer gas $(\sim 300 \mathrm{~K}$ and $\sim 2.5$ Torr) and supports a drift field of $\sim 10 \mathrm{~V} \cdot \mathrm{cm}^{-1}$. Differences in mobilities through the buffer gas cause ions to reach the second ion gate $(\mathrm{G} 2)$ at different drift times $\left(t_{D}\right)$. All pressures 
were monitored using a Baratron capacitance manometer (MKS, Andover, MA, USA). Drift time resolved mass spectra were created by applying a gating pulse to G2. The gating pulse was delayed with respect to the ion introduction pulse which set the $t_{D}$ of each mass spectrum. Because this study used analyte standard solutions rather than mixtures of analytes, the $t_{D}$ delays were scanned for the $\mathrm{m} / \mathrm{z}$ of interest corresponding to the nominal mass of the ion. Mass spectra were collected at $0.2-\mathrm{ms}$ step size intervals across the entire $t_{D}$ range of the peak $(\mathrm{m} / \mathrm{z})$ of interest. Intensities across the entire isotopic distribution were summed to provide $t_{D}$-resolved intensities. All mass spectra (.RAW files) were converted into text files (.TXT), containing the $m / z$ values and corresponding signal intensities, using the Xcalibur software suite (Version 2.2 SP1.48, ThermoScientific). The .TXT files were imported into Excel (Microsoft Cor., Redmond, WA, USA) for further analysis.

Drift time values were recorded in duplicate using two different ion gating schemes. The first scheme positions G1 at the back of F1 (Figure C1). This traps ions in the hour glass funnel prior to injection in the drift tube. The second positions G1 further in the drift tube as shown in Figure C1. Here, G1 is a true Tyndall gate. The former scheme provides significantly higher signal levels (often required for HDX) while the latter is expected to provide a more accurate $t_{D}$ value due to the precisely defined and static electric field in the gating region. That is, it is difficult to determine the effect of the RF field on the ions as they exit F1 through the first gating configuration. Therefore, the usage of the Tyndall gate serves as a cross check of collisional cross section values (see below). Because the fluorophenyl piperazine isomers were analyzed solely on the latter scheme, the analyte concentrations were increased to reflect the lower signal intensities expected with the Tyndall gate. The initial six NPSs were analyzed first with the 
gating scheme that provided higher signal levels, therefore solutions were made with a lower analyte concentration.

\subsubsection{Collisional Cross Section Calculation}

Experimental collisional cross sections $\left({ }^{\mathrm{DT}} \Omega_{\mathrm{He}}\right)$ were calculated using the following expression:

$\mathrm{DT} \Omega_{H e}=\frac{(18 \pi)^{1 / 2}}{16} \frac{z e}{\left(k_{B} T\right)^{1 / 2}}\left[\frac{1}{m_{i}}+\frac{1}{m_{b}}\right] \frac{t_{D} E}{L} \frac{760}{P} \frac{T}{273.2} \frac{1}{N}$

where $z e$ is the charge of the ion; $E$ is the electric field strength; $T$ is the temperature of the buffer gas; $k_{b}$ is Boltzmann's constant; $m_{i}$ and $m_{b}$ are the masses of the ion and the buffer gas, respectively; $L$ is the length of the drift tube; $P$ is the pressure of the buffer gas; and $N$ is the density of the buffer gas at STP. Because the $t_{D}$ window for small molecules is narrow relative to the instrument's optimum sampling step size is $(0.2 \mathrm{~ms})$, under-sampling occurs at times for some high-mobility species. For all measurements, CCS values were calculated using the signal intensity weighted centroid of the distribution.

\subsubsection{Deuterium Uptake}

For HDX experiments, $\sim 0.020$ and $\sim 0.040$ Torr of the exchange gases, $\mathrm{ND}_{3}$ and $\mathrm{D}_{2} \mathrm{O}$ respectively were added to the He buffer gas system via evaporation through a variable leak valve. Once the leak valve was opened, data were not recorded until the system's pressure stabilized to within \pm 0.005 Torr. For each mobility-selected compound the deuterium uptake level was determined for $\mathrm{D}_{2} \mathrm{O}$ and $\mathrm{ND}_{3}$ separately. Between replicate $\mathrm{HDX}$ measurements the reagent gas was removed and then reintroduced using the same leak valve settings. The number of deuterons incorporated into the molecule was determined by subtracting the average $\mathrm{m} / \mathrm{z}$ values recorded in He only from the average $\mathrm{m} / \mathrm{z}$ value after exchange. Similar to the weighted 
average $\mathrm{tD}$ calculation, each isotopologue within a user defined $\mathrm{m} / \mathrm{z}$ range was weighted by its corresponding intensity.

\subsubsection{Molecular Dynamics Simulations}

MD simulations were performed in triplicate on three disubstituted aromatic ring isomers: ortho-, meta-, and para- fluorophenyl piperazine. The simulations required the construction of singly-charged molecules, topology files, and parameter files. The fluorophenyl piperazine isomers were constructed in Avogadro ${ }^{39}$ (Version 1.2). Each isomer has two potential protonation sites (Scheme 1). Although protonation is possible on either site, the more accessible nitrogen with the higher basicity, site 1 in Scheme 1, was chosen for protonation. The initial energy minimization was performed using the Amber force field FF96 within Avogadro. Because topology and parameter files for any of the fluorophenyl piperazine isomers have not been previously reported, these files were created using the CHARMM General Force Field (CGenFF) program. Here a mol2 file of the molecule is uploaded into the program interface ${ }^{40-41}$ (Version 1.0.0). The program then performs atom typing, assigns partial atom charges, and provides parameters that includes the molecular force field ${ }^{42-43}$ (Version 3.0.1) for simulations. A psf file of the system of interest is generated using the topology files. Finally, all CHARMM parameter files, including the new CGenFF, are read into the simulation configuration files; the simulations were executed using NAMD ${ }^{44}$ (Version 2.12). A 20-ns simulation for each isomer was run in vacuo. A timestep of $0.5 \mathrm{fs}$ was used saving molecular coordinate files every 2,000 steps. Simulations were visualized and analyzed using $\operatorname{VMD}^{45}$ (Version 1.9.3) software. 


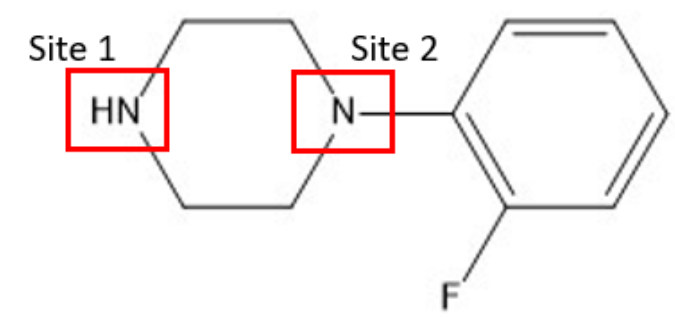

Scheme 4.1a. Structure of ortho-fluorophenyl piperazine highlighting the two potential deuterium exchange sites.

For simulations, the more basic site (site 1) was protonated.

b)

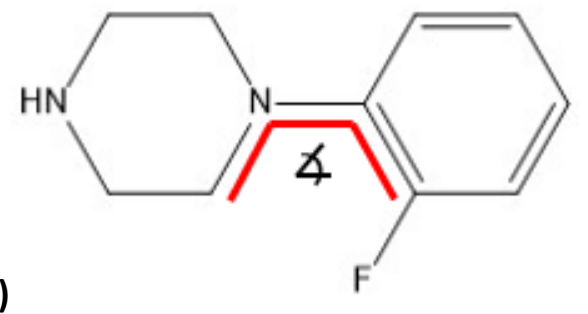

Scheme 4.1b. Example of the atoms labelled for calculation of the dihedral angle.

The dihedral angle between the two ring structures was calculated and plotted for each molecular coordinate using VMD. Because all structures were constructed in the same format, the individual atom names in the psf files were identical. The atoms forming the dihedral angle (Scheme 1) were the $\mathrm{C}_{1}, \mathrm{~N}_{1}, \mathrm{C}_{5}$, and $\mathrm{C}_{6}$ for all isomers, where the $\mathrm{C}_{6}$ atom is the carbon on the side of the aromatic ring where the fluorine is located for the various isomers. Dipole moments were calculated using the DipWatch plug-in (version 1.0) in VMD for the first simulation for each isomer. This plugin calculated the dipole moment for each molecular coordinate saved in the simulation; the dipole moment data were converted to .TXT files and imported to Excel for further analysis.

CCS values were calculated using the trajectory method ${ }^{46}$ with the Mobcal software suite ${ }^{47}$ for every $100^{\text {th }}$ structure in the first of the triplicate simulations for each isomer; this totaled 1,000 structures in which CCS values were calculated. The information contained in the original psf file was converted to a library (.lib) file. The coordinates of each sampled structure were saved as $\mathrm{XYZ}$ files (.XYZ) and then converted to an MFJ file format (.MFJ) using a custom 
MATLAB script (Version R2018a) (The MathWorks, Inc., Natick, MA, USA). The MATLAB script was written for biological molecules and was not applicable to fluorine containing molecules. To address this, the atom mass of fluorine (18.9980) was changed in the lib file to the mass of oxygen (15.9994). This allowed the MATLAB script to convert the .XYZ files to .MFJ treating the fluorine as an oxygen. Although not ideal, this conversion only affects the atomic radii (the atomic radii difference of oxygen compared to fluorine is only $\sim 2$ picometers) ${ }^{48}$ and retains the partial charge associated with the fluorine parameters. The mean CCS value was compared to the experimental value for each isomer.

\subsection{Results and Discussion}

\subsubsection{Collisional Cross Section}

The experimental parameters used for CCS calculations (Equation 4.1) are shown in Table C1. Notably, by changing the position of G1 (see above), the effective drift tube length changed from $0.99 \mathrm{~m}$ to $0.93 \mathrm{~m}$ between the first and second drift measurements, respectively, for the initial six NPSs. It was also observed that broader peaks were obtained when G1 was operated directly at the back of F1 than when G1 represents a true Tyndall gate. This may be due to the more precisely defined electric field in this region for the latter gating scheme which provides a more accurate drift time. Table 4.1 shows the ion CCS values for all compounds examined here. To our knowledge, none of the NPSs reported here have helium CCS values reported in the literature and few have been recorded for any drift gas. It is important to note that the CCS values of the three fluorophenyl piperazine isomers are nearly identical making them indistinguishable by IMS and MS alone. 
Table 4.1.CCS values and deuterium uptake for all compounds.

\begin{tabular}{|c|c|c|c|c|c|}
\hline \multirow{2}{*}{ Compound $^{1}$} & \multirow{2}{*}{$\begin{array}{l}\text { M.W. } \\
(\mathrm{g} / \mathrm{mol})\end{array}$} & \multirow{2}{*}{$\begin{array}{l}\text { Amine } \\
\text { Groups }\end{array}$} & \multirow{2}{*}{$\begin{array}{l}\text { CCS } \\
\left(\dot{\mathrm{A}}^{2}\right)^{2}\end{array}$} & \multicolumn{2}{|c|}{ Deuterium Uptake 3} \\
\hline & & & & $\mathbf{D}_{2} \mathbf{O}$ & $\mathrm{ND}_{3}$ \\
\hline 4-fluoroamphetamine & 154 & $1^{\circ}$ & $68(3)$ & $0.09(3.53 \mathrm{E}-2)$ & 2.54 (7.36E-1) \\
\hline Benzyl piperazine & 177 & $2^{\circ}, 3^{\circ}$ & $76(2)$ & $1.07(6.29 \mathrm{E}-2)$ & $1.85(2.01 \mathrm{E}-1)$ \\
\hline 5-MeO-AMT & 205 & $1^{\circ}, 2^{\circ}$ & $82(2)$ & $0.15(6.78 \mathrm{E}-2)$ & $3.43(4.48 \mathrm{E}-1)$ \\
\hline Methylone & 208 & $2^{\circ}$ & $78(2)$ & $0.16(3.46 \mathrm{E}-2)$ & $1.84(4.00 \mathrm{E}-2)$ \\
\hline AH-79 & 329 & $2^{\circ}, 3^{\circ}$ & $107(4)$ & $0.02(1.07 \mathrm{E}-1)$ & $0.30(4.02 \mathrm{E}-2)$ \\
\hline 25C-NBOMe & 336 & $2^{\circ}$ & $112(0)$ & $0.02(2.27 \mathrm{E}-2)$ & $1.99(3.96 \mathrm{E}-2)$ \\
\hline & 181 & & $77(1)$ & $1.79(4.80 \mathrm{E}-3)$ & $1.90(6.13 \mathrm{E}-2)$ \\
\hline Meta & 181 & $2^{\circ}, 3^{\circ}$ & $78(0)$ & $1.85(1.15 \mathrm{E}-2)$ & $1.89(4.62 \mathrm{E}-3)$ \\
\hline Para & 181 & $2^{\circ}, 3^{\circ}$ & $77(1)$ & $1.85(7.39 \mathrm{E}-3)$ & $1.84(4.14 \mathrm{E}-3)$ \\
\hline
\end{tabular}

${ }^{1}$ The ortho, meta, and para indicate fluorophenyl piperazine isomers.

${ }^{2}$ Differences in CCS values determined by the duplicate measurements are noted parenthetically.

${ }^{3}$ Two Standard deviations, estimate of $95 \%$ confidence interval $(n=3)$, are shown parenthetically.

\subsubsection{HDX Reactivities}

The exchange mechanisms of the reagent gases were examined in the 1990's and it was suggested that exchange occurs via different mechanisms. ${ }^{49}$ Deuterium exchange with a $\mathrm{D}_{2} \mathrm{O}$ molecule occurs via the 'relay mechanism' in which the protonation site and an additional basic site on the analyte must be in close proximity for the transfer to occur. The proton from the charge site is transferred to the $\mathrm{D}_{2} \mathrm{O}$ molecule as a deuteron is shuttled to a less basic site on the analyte. Therefore, for the exchange to occur, a long-lived reaction complex must be formed near the exchange site. Conversely, exchange involving $\mathrm{ND}_{3}$ as the reagent molecule occurs via an 'onium ion mechanism'. Simultaneously the deuterated reagent onium is solvated, and the deuteron is exchanged to the available site on the analyte. Comparatively, the onium ion exchange process is more efficient than the relay mechanism and is, thus, possibly less discriminate. ${ }^{49}$

An initial evaluation of the HDX propensity was conducted for six compounds (Table 4.1 top) encompassing a variety of heteroatom sites that are potential sites of exchange (i.e. single or multiple amines and primary, secondary, and tertiary amines) and structural features 
(i.e. amines within ring structures, terminal amines, etc.). Five of the six molecules showed minimal to no exchange when $\mathrm{D}_{2} \mathrm{O}$ was the reagent gas (Figures 4.1 and C2a-e). Methylone, for example, showed minimal deuterium uptake with $\mathrm{D}_{2} \mathrm{O}$ as evidenced by a slight increase in the abundance of the $\mathrm{D}_{1}$ and subsequent isotopologue peaks (Figure C2c). Five of the six molecules showed more significant exchange in the presence of $\mathrm{ND}_{3}$. Interestingly, 5-MeO-AMT contained four heteroatom hydrogens on two separate amine sites (Figure C2e); this compound showed significant ion populations undergoing three and four exchanges in the presence of $\mathrm{ND}_{3}$. Exchange on both amine sites suggests that exchange with $\mathrm{ND}_{3}$ is less discriminate, exchanging all possible labile heteroatom hydrogens. On the other hand, AH-7921 showed minimal exchange in the presence of either reagent gas (Figure Cd). AH-72921 contains both a tertiary amine and an amide; both groups are near bulky structures within the molecule which may reduce the accessibility of the reagent gas to the exchange site. The inability to exchange with $\mathrm{ND}_{3}$, despite $\mathrm{ND}_{3}$ being indiscriminate as a reagent gas (as evidenced by the exchange with 5MeO-AMT), suggests that this is case. Average deuterium uptake values and the estimation of the 95\% confidence interval are reported for all mobility selected compounds in Table 4.1.

Benzyl piperazine $[\mathrm{M}+\mathrm{H}]^{+}$ions were the only ions, of the initial 6 compounds, showing appreciable exchange with $\mathrm{D}_{2} \mathrm{O}$ (Figure 4.1). This is shown by the decreased abundance of the $\mathrm{D}_{0}$ isotopologue and the increased abundance of the $\mathrm{D}_{1}$ and $\mathrm{D}_{2}$ ions. Compared to $\mathrm{ND}_{3}$, exchange in the presence of $\mathrm{D}_{2} \mathrm{O}$ was less efficient as the $\mathrm{D}_{0}$ peak persists to a much greater extent. It was hypothesized that the unique diamine ring in the compound allows exchange in the presence of $\mathrm{D}_{2} \mathrm{O}$. Because the nitrogen atoms are fixed in the ring structure, the proximity requirement of the site of protonation and the charged site is relatively ensured. Therefore, the diamine ring structure more likely favors the formation of the long-lived reaction complex. 


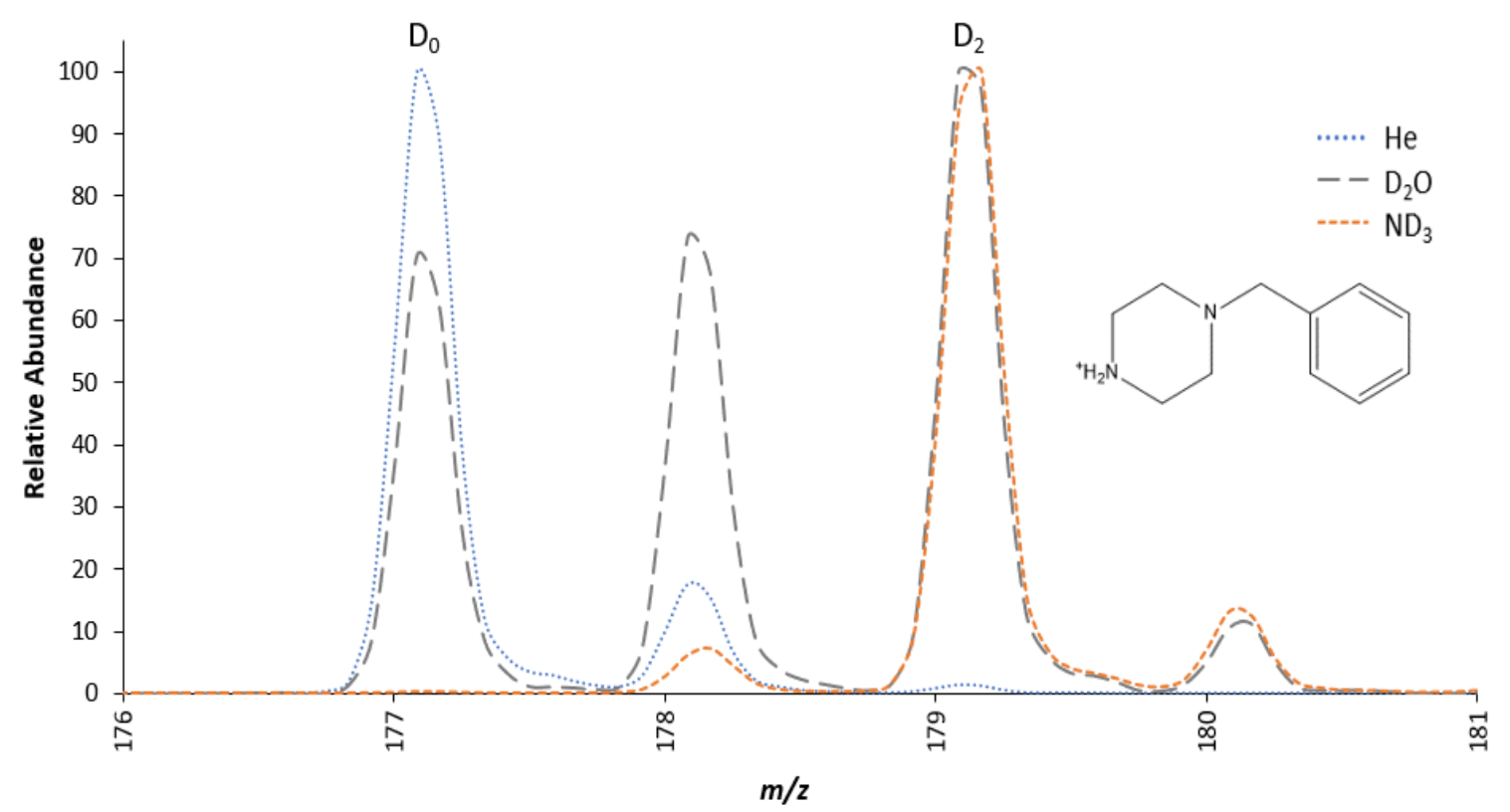

Figure 4.1. Isotopic distributions for benzyl piperazine.

For reference, the $\mathrm{D}_{0}$ and $\mathrm{D}_{2}$ isotopologue peaks are labeled and the protonated molecular structures are provided as insets.

\subsubsection{Discrimination of Disubstituted Aromatic Ring Isomers}

The fluorophenyl piperazine isomers were chosen for this study because they contained the diamine ring enabling $\mathrm{HDX}$ with $\mathrm{D}_{2} \mathrm{O}$ and the standards were available in all three isomer positions (ortho-, meta-, and para-). When the isomers were in the presence of the drift gas (He), isotopic distributions are indiscernible, as shown in Figure C3. The analysis of each isomer after reacting with $\mathrm{D}_{2} \mathrm{O}$ and $\mathrm{ND}_{3}$ revealed small variations in the isotopic distributions indicating that there were slight differences in the deuterium uptake (Figures 4.2a and 4.2b). Because the relative abundance of the $\mathrm{D}_{1}$ ion peak for the ortho isomer during $\mathrm{D}_{2} \mathrm{O}$ analysis is larger ( 25\%) compared to the para isomer ( $14 \%)$, it appears that these ions undergo fewer exchange events (Figure 4.2a). Indeed, the average deuterium uptake values are observed to be 1.79 and 1.85 for the ortho and para isomers, respectively (Table 4.1 bottom). Figure $4.2 \mathrm{~b}$ shows that this is reversed for the $\mathrm{D}_{1}$ ion peak when $\mathrm{ND}_{3}$ is used as the reagent gas. On average, the ortho and para 
isomers exhibit 1.90 and 1.84 deuterium uptake, respectively, when $\mathrm{ND}_{3}$ is used as the reagent gas (Table 4.1 bottom). Finally, it is noted that the use of $\mathrm{ND}_{3}$ results in greater exchange for many of the ions studied here. This may be expected as exchange with $\mathrm{ND}_{3}$ does not rely on the 'relay mechanism'. ${ }^{49}$

It is also of interest to note the mass shift that occurs with the $\mathrm{D}_{1}$ ion peak for ortho isomer in the presence of both reagent gases and, to a lesser extent, with the meta ions in the presence of $\mathrm{D}_{2} \mathrm{O}$ (Figures 4.2a and 4.2b). These mass shifts have been known to occur with ion trap mass spectrometers for ions of with specific physiochemical properties, namely easily polarizable ${ }^{50}$ and permanent dipole moments ${ }^{51}$. The theoretical dipole moments of the three isomers were calculated (Table C2); all three fluorophenyl piperazine isomers studied have permanent dipole moments indicating the isomers are polarizable, albeit to different degrees. The ortho isomer has a smaller dipole moment compared to meta and para. The observed mass shift is thought to be the result of the permanent dipole moment and the polarizability differences of the isomers. 


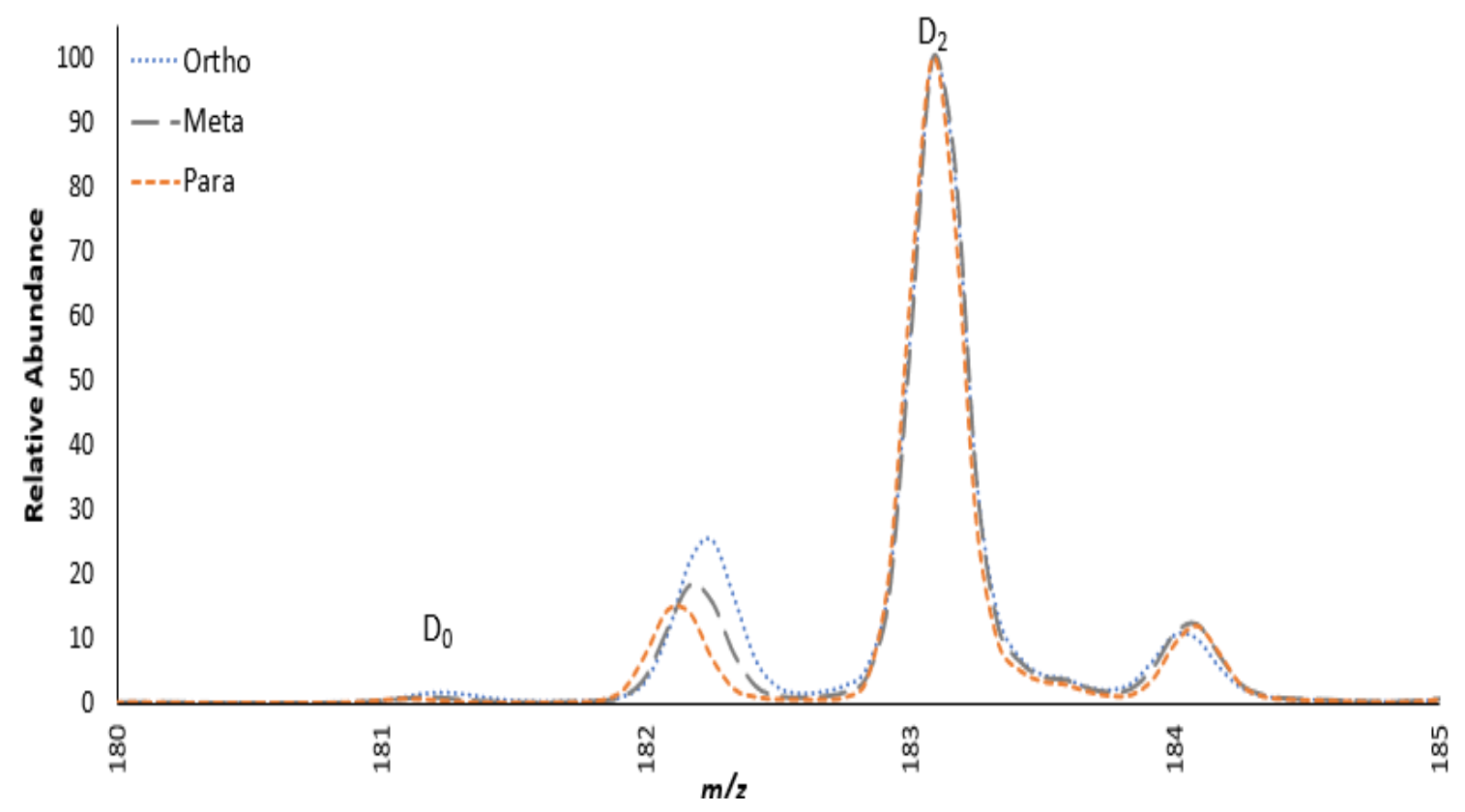

Figure 4.2a. Isotopic distributions for all fluorophenyl piperazine isomers when $\mathrm{D}_{2} \mathrm{O}$ is used as the reagent gas.

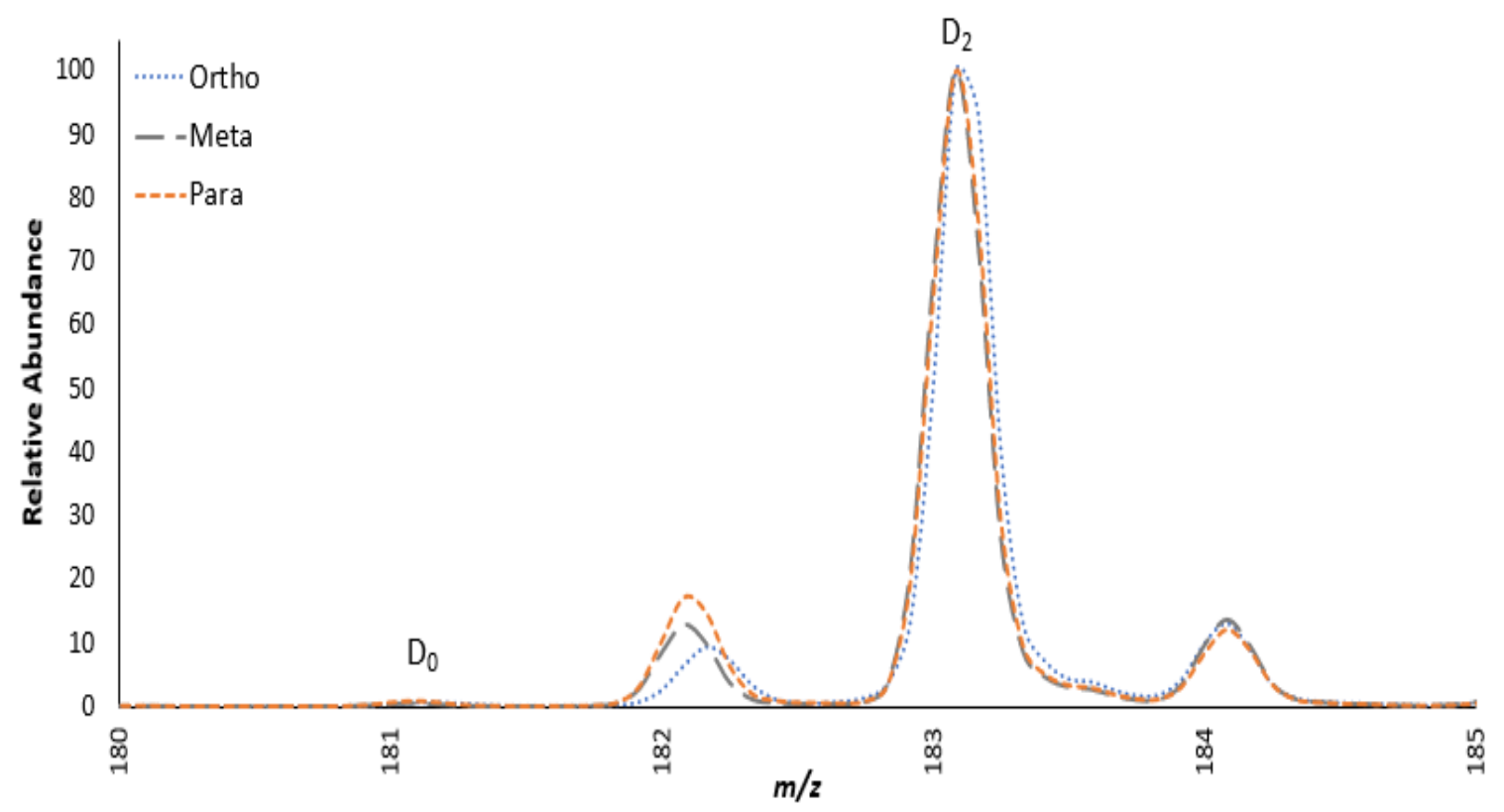

Figure 4.2b. Isotopic distributions for all fluorophenyl piperazine isomers when $\mathrm{ND}_{3}$ is used as the reagent gas.

With the deuterium uptake values, a question arises as to the utility in distinguishing the fluorophenyl piperazine isomers. As shown in Figure C3, the $\mathrm{D}_{2} \mathrm{O}$ deuterium uptake of the meta 
and para isomers are nearly identical, as is the $\mathrm{ND}_{3}$ deuterium uptake for the ortho and meta isomers. Each sample measurement, i.e. each isomer with each reagent gas, was recorded in triplicate $(n=3)$. A Tukey means comparison was performed using Origin (OriginLab, North Hampton, MA) at the $\alpha=0.05$ level shows that the meta-para $\mathrm{D}_{2} \mathrm{O}$ comparison and the ortho-meta $\mathrm{ND}_{3}$ comparison are not significantly different $(\mathrm{p}=0.97$ and $\mathrm{p}=0.87$, respectively). All other comparison pairings for both $\mathrm{D}_{2} \mathrm{O}$ and $\mathrm{ND}_{3}$ are significantly different with p-values in the E-6 to E-2 range. In short, the data from individual reagent gases does not necessarily allow for the discrimination of the fluorophenyl piperazine isomers but the combined data provides complete isomer discrimination at the $95 \%$ confidence interval. Additional studies should be performed to ensure inter-instrumentation discrimination; however, the data presented here demonstrates the ability to confidently discriminate between isomers with the current experimental setup.

The causes of the observed HDX differences when comparing reagent gases are difficult to elucidate. However, it was hypothesized that the substitution position on the aromatic ring within the fluorophenyl piperazine isomers could play a role in HDX kinetics. For exchange with $\mathrm{D}_{2} \mathrm{O}$, for example, the electronegative fluorine atom at the ortho position would render the shuttle nitrogen less basic suggesting less facile initial deuterium incorporation. For the para and meta isomers, this effect could be somewhat diminished resulting in higher efficiency of initial deuterium incorporation. For $\mathrm{HDX}$ with $\mathrm{ND}_{3}$, the stabilization of the onium ion intermediate and closer proximity of the fluorine constituent for the ortho and meta isomers could lead to increased exchange for these ions.

\subsubsection{Molecular Dynamics Simulations}

Molecular dynamics (MD) simulations were performed to determine if deuterium uptake differences could be further elucidated in light of structural fluctuations. In general, the 
theoretical CCS values were in good agreement with experimental values for all three fluorophenyl piperazine isomers (Table C2). The dihedral angle of each isomer, as defined in Scheme 1, was tracked for each replicate simulation (Figure 4.3). The meta and para isomer dihedral angles behaved somewhat as expected- angle populations at $\pm 0^{\circ}, 60^{\circ}$, and $180^{\circ}$. The ortho isomer, conversely, shows that the dihedral angle favors $<0^{\circ}$. The observance of values above $0^{\circ}$ suggests that although it is possible for the two rings to rotate a full $360^{\circ}$, angles $>0^{\circ}$ are less favorable. This was observed for all 3 simulations (Figure C5a). Essentially the ring structures favored an orthogonal positioning to one another, creating a 'limited angle' for rotation. This appears to result from the close proximity of the fluorine to the diamine ring.

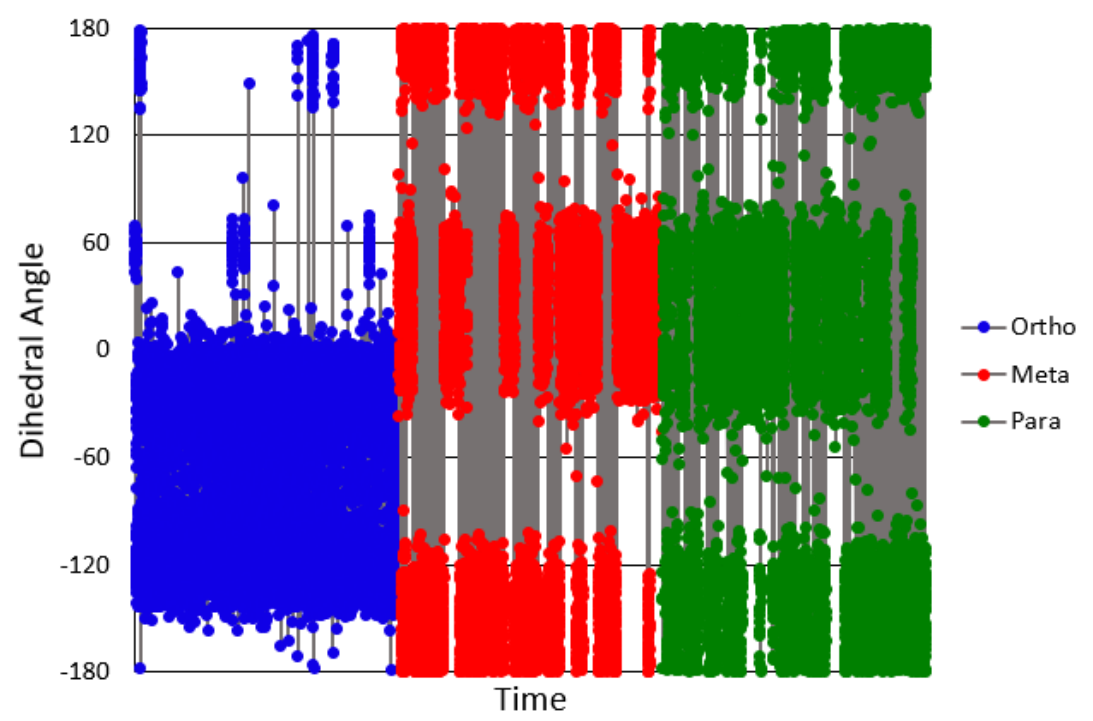

Figure 4.3. Dihedral angle between the aromatic ring and diamine ring for all fluorophenyl piperazine isomers.

The first simulation is shown for all isomers. The $\mathrm{x}$-axis is time, i.e. where each isomer starts at 0 ns and finishes at $20 \mathrm{~ns}$.

The reduced deuterium uptake from $\mathrm{D}_{2} \mathrm{O}$ of the ortho isomer, compared to the other isomers, may result from a hindrance to formation by this more rigid system. For example, the exchange complex formation may be destabilized on the side of the diamine ring in which the fluorine resides. The rigid system may also contribute to the larger error bars noted with the 
ortho isomer in the presence of $\mathrm{ND}_{3}$. This is in contrast to the meta and para isomers, where all naturally favored dihedral angles are sufficiently represented in the MD simulations signifying that these isomers experience a 'free' rotation between the ring systems. Such an explanation of rotation cannot be invoked for exchange by $\mathrm{ND}_{3}$, as the meta and para isomers exhibit different exchange levels. Rather, as mentioned above, the proximity of the fluorine may impart better stabilization of the onium ion complex for the former isomer.

\subsection{Conclusions}

Of the six NPSs initially evaluated for exchange propensity, one compound did not exchange with either reagent gas. It is hypothesized that the bulky structures surrounding the amine sites in AH-7921 reduce the accessibility of the reagent gas to the exchange site resulting in minimal exchange. Only one of the initial NPSs exchanged with both reagent gases, benzyl piperazine. The diamine ring of this NPS, and of the fluorophenyl piperazine isomers subsequently studied, provided a fixed length between the exchange site and the charge site. This likely facilitated the exchange with $\mathrm{D}_{2} \mathrm{O}$, which, despite being less efficient than $\mathrm{ND}_{3}$, was capable of exchange with at least one labile heteroatom hydrogen. A NPS capable of exchange in the presence of $\mathrm{D}_{2} \mathrm{O}$ suggests that a fixed length/position, or nearly fixed position, between the exchange position and the additional charge site is present. Structural inferences like these, can aid in compound identification.

Despite selecting NPSs with potentially labile heteroatom hydrogens, five of the nine NPSs studied did not exchange with $\mathrm{D}_{2} \mathrm{O}$. It is also important to note that NPSs without heteroatoms (which would not contain potentially labile hydrogens) have been identified. This novel application, however, demonstrates that discrimination is possible if the compound exchanges in the presence of both reagent gases. Even though IMS and MS is not capable of 
distinguishing the ortho-, meta-, and para-fluorophenyl piperazine isomers here, HDX is capable of discrimination at a $95 \%$ confidence interval with the current instrumentation. These findings suggest gas-phase HDX can add a level of discrimination for non-targeted, small molecule analysis.

Future work has several avenues of interest. Studies analyzing various piperazines, containing the diamine ring, with different halogens and/or substitutions will be pursued. This would identify if isomer discrimination with less electronegative substitutions is possible and will determine how substituents affect HDX on small synthetic molecules. As a final note, an inter-laboratory study should be conducted to evaluate HDX isomer discrimination among instruments and laboratories.

\subsection{Funding and Acknowledgments}

S. Valentine is grateful for support from the National Science Foundation (CHE1553021) for this work. This work was also supported, in part, by the United States Department of Commerce National Institute of Standards and Technology Award \# 70NANB16H105. The authors wish to thank Sandra Majuta for her help with calculating theoretical collisional cross sections.

\subsection{References}

1. Namera, A., Comprehensive review of the detection methods for synthetic cannabinoids and cathinones. Forensic Toxicology 2015, 33 (2), 175.

2. Pasin, D.; Cawley, A.; Bidny, S.; Fu, S., Current applications of high-resolution mass spectrometry for the analysis of new psychoactive substances: a critical review. Analytical and Bioanalytical Chemistry 2017, 409 (25), 5821-5836. 
3. Controlled Substances Act: Treatment of Controlled substance Analogues. In 21, 21 ed.; United States: 1986; Vol. 13B.

4. Zawilska, J. B.; Andrzejczak, D., Next generation of novel psychoactive substances on the horizon - A complex problem to face. Drug and Alcohol Dependence 2015, 157, 1-17.

5. Smith, J. P., An overview of recent developments in the analytical detection of new psychoactive substances (NPSs). Analyst 2015, 140 (15), 4932.

6. Kanu, A. B.; Brandt, S. D.; Williams, M. D.; Zhang, N.; Hill, H. H., Analysis of psychoactive cathinones and tryptamines by electrospray ionization atmospheric pressure ion mobility time-of-flight mass spectrometry. Analytical chemistry 2013, 85 (18), 8535-8542.

7. Midey, A. J.; Patel, A.; Moraff, C.; Krueger, C. A.; Wu, C., Improved detection of drugs of abuse using high-performance ion mobility spectrometry with electrospray ionization (ESIHPIMS) for urine matrices. Talanta 2013, 116, 77-83.

8. Joshi, M.; Cetroni, B.; Camacho, A.; Krueger, C.; Midey, A. J., Analysis of synthetic cathinones and associated psychoactive substances by ion mobility spectrometry. Forensic science international 2014, 244, 196-206.

9. Sysoev, A.; Poteshin, S.; Chernyshev, D.; Karpov, A.; Tuzkov, Y.; Kyzmin, V.; Sysoev, A., Analysis of new synthetic drugs by ion mobility time-of-flight mass spectrometry. European Journal of Mass Spectrometry 2014, 20 (2), 185-192.

10. Armenta, S.; Garrigues, S.; Guardia, M.; Brassier, J.; Alcalà, M.; Blanco, M.; PerezAlfonso, C.; Galipienso, N., Detection and characterization of emerging psychoactive substances by ion mobility spectrometry. Drug testing and analysis 2015, 7 (4), 280-289. 
11. Gwak, S.; Almirall, J. R., Rapid screening of 35 new psychoactive substances by ion mobility spectrometry (IMS) and direct analysis in real time (DART) coupled to quadrupole time-of-flight mass spectrometry (QTOF-MS). Drug Test. Anal. 2015, 7 (10), 884-893.

12. Majuta, S. N.; Maleki, H.; Kiani Karanji, A.; Attanyake, K.; Loch, E.; Valentine, S. J., Magnifying ion mobility spectrometry-mass spectrometry measurements for biomolecular structure studies. Current Opinion in Chemical Biology 2018, 42, 101-110.

13. Donohoe, G. C.; Maleki, H.; Arndt, J. R.; Khakinejad, M.; Yi, J.; McBride, C.; Nurkiewicz, T. R.; Valentine, S. J., A new ion mobility-linear ion trap instrument for complex mixture analysis. Analytical chemistry 2014, 86 (16), 8121-8128.

14. Stow, S. M.; Onifer, T. M.; Forsythe, J. G.; Nefzger, H.; Kwiecien, N. W.; May, J. C.; McLean, J. A.; Hercules, D. M., Structural Characterization of Methylenedianiline Regioisomers by Ion Mobility-Mass Spectrometry, Tandem Mass Spectrometry, and Computational Strategies. 2. Electrospray Spectra of 3-Ring and 4-Ring Isomers. Analytical chemistry 2015, 87 (12), 62886296.

15. Werres, T.; Leonhardt, J.; Jäger, M.; Teutenberg, T., Critical Comparison of Liquid Chromatography Coupled to Mass Spectrometry and Three Different Ion Mobility Spectrometry Systems on Their Separation Capability for Small Isomeric Compounds. Chromatographia 2019, $82(1), 251-260$.

16. Ouyang, H.; Bo, T.; Zhang, Z.; Guo, X.; He, M.; Li, J.; Yang, S.; Ma, X.; Feng, Y., Ion mobility mass spectrometry with molecular modelling to reveal bioactive isomer conformations and underlying relationship with isomerization. Rapid Communications in Mass Spectrometry 2018, 32 (22), 1931-1940. 
17. Kune, C.; Haler, J. R.; Far, J.; De Pauw, E., Effectiveness and limitations of computational chemistry and mass spectrometry in the rational design of target-specific shift reagents for ion mobility spectrometry. ChemPhysChem 2018, 19 (21), 2921-2930.

18. Mu, Y.; Schulz, B.; Ferro, V., Applications of Ion Mobility-Mass Spectrometry in Carbohydrate Chemistry and Glycobiology. Molecules 2018, 23 (10), 2557.

19. Harvey, D. J.; Struwe, W. B., Structural Studies of Fucosylated N-Glycans by Ion Mobility Mass Spectrometry and Collision-Induced Fragmentation of Negative Ions. Journal of The American Society for Mass Spectrometry 2018, 29 (6), 1179-1193.

20. Tsai, C. W.; Tipple, C. A.; Yost, R. A., Integration of paper spray ionization high-field asymmetric waveform ion mobility spectrometry for forensic applications. Rapid Communications in Mass Spectrometry 2018, 32 (7), 552-560.

21. Chouinard, C. D.; Nagy, G.; Webb, I. K.; Shi, T.; Baker, E. S.; Prost, S. A.; Liu, T.; Ibrahim, Y. M.; Smith, R. D., Improved Sensitivity and Separations for Phosphopeptides using Online Liquid Chromotography Coupled with Structures for Lossless Ion Manipulations Ion Mobility-Mass Spectrometry. Analytical chemistry 2018, 90 (18), 10889-10896.

22. Englander, S. W., Hydrogen exchange and mass spectrometry: A historical perspective. Journal of the American Society for Mass Spectrometry 2006, 17 (11), 1481-1489.

23. Konermann, L.; Vahidi, S.; Sowole, M. A., Mass spectrometry methods for studying structure and dynamics of biological macromolecules. Analytical chemistry 2013, 86 (1), 213232.

24. Masson, G. R.; Jenkins, M. L.; Burke, J. E., An overview of hydrogen deuterium exchange mass spectrometry (HDX-MS) in drug discovery. Expert opinion on drug discovery 2017, 12 (10), 981-994. 
25. Marciano, D. P.; Dharmarajan, V.; Griffin, P. R., HDX-MS guided drug discovery: small molecules and biopharmaceuticals. Current opinion in structural biology 2014, 28, 105-111.

26. Lee, J.-J.; Park, Y. S.; Lee, K.-J., Hydrogen-deuterium exchange mass spectrometry for determining protein structural changes in drug discovery. Archives of pharmacal research 2015, $38(10), 1737-1745$.

27. Liu, D. Q.; Hop, C. E.; Beconi, M. G.; Mao, A.; Chiu, S. H. L., Use of on-line hydrogen/deuterium exchange to facilitate metabolite identification. Rapid Communications in Mass Spectrometry 2001, 15 (19), 1832-1839.

28. Ohashi, N.; Furuuchi, S.; Yoshikawa, M., Usefulness of the hydrogen-deuterium exchange method in the study of drug metabolism using liquid chromatography-tandem mass spectrometry. Journal of pharmaceutical and biomedical analysis 1998, 18 (3), 325-334.

29. Lam, W.; Ramanathan, R., In electrospray ionization source hydrogen/deuterium exchange LC-MS and LC-MS/MS for characterization of metabolites. Journal of the American Society for Mass Spectrometry 2002, 13 (4), 345-353.

30. Suckau, D.; Shi, Y.; Beu, S. C.; Senko, M. W.; Quinn, J. P.; Wampler, F.; McLafferty, F. W., Coexisting stable conformations of gaseous protein ions. Proceedings of the National Academy of Sciences 1993, 90 (3), 790-793.

31. Valentine, S. J.; Clemmer, D. E., H/D exchange levels of shape-resolved cytochrome c conformers in the gas phase. Journal of the American Chemical Society 1997, 119 (15), 35583566.

32. Freitas, M.; Marshall, A., Rate and extent of gas-phase hydrogen/deuterium exchange of bradykinins: evidence for peptide zwitterions in the gas phase1. International journal of mass spectrometry 1999, 182, 221-231. 
33. Rand, K. D.; Pringle, S. D.; Murphy III, J. P.; Fadgen, K. E.; Brown, J.; Engen, J. R., Gas-phase hydrogen/deuterium exchange in a traveling wave ion guide for the examination of protein conformations. Analytical chemistry 2009, 81 (24), 10019-10028.

34. Khakinejad, M.; Kondalaji, S. G.; Tafreshian, A.; Valentine, S. J., Comprehensive gasphase peptide ion structure studies using ion mobility techniques: part 2. Gas-phase hydrogen/deuterium exchange for ion population estimation. Journal of The American Society for Mass Spectrometry 2017, 28 (5), 960-970.

35. Molano-Arevalo, J. C.; Jeanne Dit Fouque, K.; Pham, K.; Miksovska, J.; Ridgeway, M. E.; Park, M. A.; Fernandez-Lima, F., Characterization of intramolecular interactions of cytochrome c using hydrogen-deuterium exchange-trapped ion mobility spectrometry-mass spectrometry and molecular dynamics. Analytical chemistry 2017, 89 (17), 8757-8765.

36. Khakinejad, M.; Kondalaji, S. G.; Maleki, H.; Arndt, J. R.; Donohoe, G. C.; Valentine, S. J., Combining ion mobility spectrometry with hydrogen-deuterium exchange and top-down MS for peptide ion structure analysis. Journal of the American Society for Mass Spectrometry 2014, 25 (12), 2103-2115.

37. Maleki Hossein, H.; Karanji, A. K.; Majuta, S.; Maurer, M. M.; Valentine, S. J., Ion Mobility Spectrometry-Mass Spectrometry Coupled with Gas-Phase Hydrogen/Deuterium Exchange for Metabolomics Analyses. Journal of The American Society for Mass Spectrometry 29 (2), 230-241.

38. Uppal, S. S.; Beasley, S. E.; Scian, M.; Guttman, M., Gas-Phase Hydrogen/Deuterium Exchange for Distinguishing Isomeric Carbohydrate Ions. Analytical Chemistry 2017, 89 (8), 4737-4742. 
39. Hanwell, M. D.; Curtis, D. E.; Lonie, D. C.; Vandermeersch, T.; Zurek, E.; Hutchison, G. R., Avogadro: an advanced semantic chemical editor, visualization, and analysis platform. Journal of Cheminformatics 2012, 4 (1), 17.

40. Vanommeslaeghe, K.; Hatcher, E.; Acharya, C.; Kundu, S.; Zhong, S.; Shim, J.; Darian, E.; Guvench, O.; Lopes, P.; Vorobyov, I., CHARMM general force field: A force field for druglike molecules compatible with the CHARMM all-atom additive biological force fields. Journal of computational chemistry 2010, 31 (4), 671-690.

41. Yu, W.; He, X.; Vanommeslaeghe, K.; MacKerell, A. D., Extension of the CHARMM general force field to sulfonyl-containing compounds and its utility in biomolecular simulations. Journal of computational chemistry 2012, 33 (31), 2451-2468.

42. Vanommeslaeghe, K.; MacKerell Jr, A. D., Automation of the CHARMM General Force Field (CGenFF) I: bond perception and atom typing. Journal of chemical information and modeling 2012, 52 (12), 3144-3154.

43. Vanommeslaeghe, K.; Raman, E. P.; MacKerell Jr, A. D., Automation of the CHARMM General Force Field (CGenFF) II: assignment of bonded parameters and partial atomic charges. Journal of chemical information and modeling 2012, 52 (12), 3155-3168.

44. Phillips, J. C.; Braun, R.; Wang, W.; Gumbart, J.; Tajkhorshid, E.; Villa, E.; Chipot, C.; Skeel, R. D.; Kale, L.; Schulten, K., Scalable molecular dynamics with NAMD. Journal of computational chemistry 2005, 26 (16), 1781-1802.

45. Humphrey, W.; Dalke, A.; Schulten, K., VMD: visual molecular dynamics. Journal of molecular graphics 1996, 14 (1), 33-38. 
46. Mesleh, M.; Hunter, J.; Shvartsburg, A.; Schatz, G. C.; Jarrold, M., Structural information from ion mobility measurements: effects of the long-range potential. The Journal of Physical Chemistry 1996, 100 (40), 16082-16086.

47. Group, J. R. MOBCAL- A Program to Calculate Mobilities, Indiana University.

48. Gilbert, T. R.; Kirss, R. V.; Foster, N.; Davies, G., Chemistry: The science in context. WW Norton \& Company: 2013.

49. Campbell, S.; Rodgers, M.; Marzluff, E. M.; Beauchamp, J., Deuterium exchange reactions as a probe of biomolecule structure. Fundamental studies of gas phase H/D exchange reactions of protonated glycine oligomers with D2O, CD3OD, CD3CO2D, and ND3. Journal of the American Chemical Society 1995, 117 (51), 12840-12854.

50. Traldi, P.; Favretto, D.; Catinella, S.; Bortolini, O., Mass displacements in quadrupolar field analysers. Organic mass spectrometry 1993, 28 (7), 745-751.

51. Bortolini, O.; Spalluto, G.; Traldi, P., Relationship between mass displacements and dipole moments of para-substituted pyridine odd-electron molecular ions. Organic mass spectrometry 1994, 29 (5), 269-271. 


\section{Chapter 5: Conclusions and Future Directions}

\subsection{Conclusions}

Forensic drug chemistry is facing a unique and challenging environment created by the never-ending cycle of NPSs entering the drug market. Without standards, drug chemists are performing non-target analyses more often and relying on mass spectral library searches for identifications. During an initial evaluation of the inherent variation in GCMS mass spectra, it was shown that without frequent tuning the variation can be substantial ( $\leq 250 \% \mathrm{RSD})$. An additional source of uncertainty in GCMS identifications can result from inconsistent base peaks either within or between instruments. Because the signal intensity in mass spectra are reported relative to the most abundant peak, an inconsistent base peak within the same instrument results in increased uncertainty of all \%RAs while an inconsistent base peak between two instruments hinders mass spectral comparisons. Although employed for decades, passing the EPA's DFTPP criteria was not correlated with reduced variation, rather tuning frequency was associated with a reduction in ion \%RA variation. With frequent tuning, i.e. immediately prior to analysis, variation was reduced 5-fold. Finally, it was noted that variation in \%RAs is not independent of fragmentation patterns; however, thorough evaluation of this dependence was not possible with the current data set.

As a novel approach to non-target NPS analysis, HDX-IMS-MS was evaluated in a proof of concept study for the potential discrimination of isomers with minute structural differences. Ortho-, meta-, and para- fluorophenyl piperazine isomer discrimination was feasible at a 95\% CI with the current instrumentation when the data from both reagent gases was considered. Despite selecting NPSs with potentially labile heteroatom hydrogens, five of the six NPSs did not exchange with $\mathrm{D}_{2} \mathrm{O}$. A fixed length between the chargeable site and the exchange site was linked 
to the ability to exchange with $\mathrm{D}_{2} \mathrm{O}$; this observation suggests that non-targeted HDX analyses can allow for additional structural inferences. One NPS did not exchange in the presence of either reagent gas, AH-7921. Because both potential exchange sites are surrounded by bulky structures within the molecule, the lack of exchange in the presence of either reagent gas was likely due to inaccessible exchange sites. Although NPSs with potentially labile hydrogens were chosen for the study, the low exchange reactivity in the presence of either reagent gas is a clear limitation of the technique, as data from both reagent gases were required for isomer discrimination.

\subsection{Future Directions}

Three primary areas of interest remain for the evaluation of GCMS uncertainty. First, it would useful to investigate the frequency with which a library search returns the correct identification and, when incorrect, how often the correct NPS family is identified. This could be studied by analyzing NPSs, both with and without structurally similar analogues, known to be in the mass spectral library and conducting non-targeted library searches. Varying the structural similarity of the analytes will help identify how closely the search algorithm returns the correct NPS family. It would also be prudent to analyze all databases relevant to forensic drug chemistry including NIST, SWGDRUG, and Wiley Mass Spectra Database of Designer Drugs. Second, because the uncertainty of ion \%RAs was found to be dependent, to an unknown degree, on fragmentation, it is possible that utilizing EPA tuning criteria is not fit for purpose. This can be investigated by comparing the ion \%RA variation of DFTPP/BFB to NPSs; if the variation is sufficiently different, it may suggest that this tune check compound is not suitable for limiting the variation of NPSs. A study identifying a more relevant and fit for purpose tune check compound could be performed with the aim of developing a tune check criterion specifically for 
NPSs. Finally, compounds that produce the same $\mathrm{m} / \mathrm{z}$ value but have different structural features should be analyzed as an initial step to evaluate the dependence of ion \%RA variation on fragmentation patterns. This information is vital in order to calculate of the inherent uncertainty of GCMS analyses.

Because the HDX-IMS-MS study was proof of concept, there are a few research avenues which could be pursued and should focus on further evaluating the utility of HDX for small molecule non-target analyses. The first avenue to be pursued should be an interlaboratory study of isomer discrimination. Although the current instrumentation was shown to be suitable for isomer discrimination, other instrumentation should also be evaluated. Next, various phenyl piperazines with other halogen substitutions should be studied. Less electronegative halogens will undoubtedly affect the exchange reactivities, therefore, isomer discrimination should be evaluated with a variety of substitutions. The sum of these studies will provide a thorough basis evaluating the utility of this technique for isomer discrimination. 


\section{Appendix A. \\ Chapter 2: Evaluation of the Reproducibility and Repeatability of GCMS Retention Indices and Mass Spectra of Novel Psychoactive Substances - Supplemental Information}

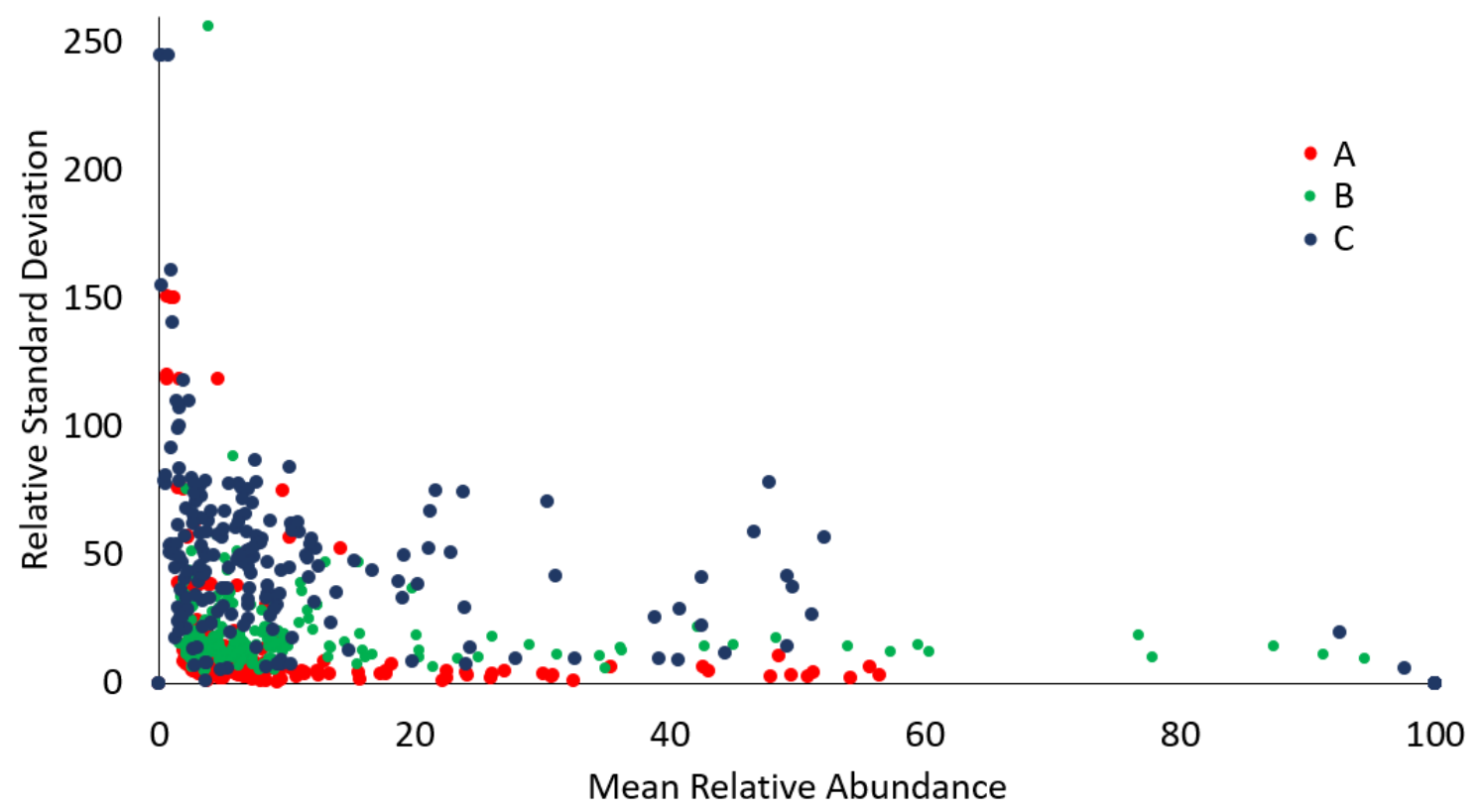

Figure A1a. \% RSD plotted as a function of mean \%RA for all three instruments.

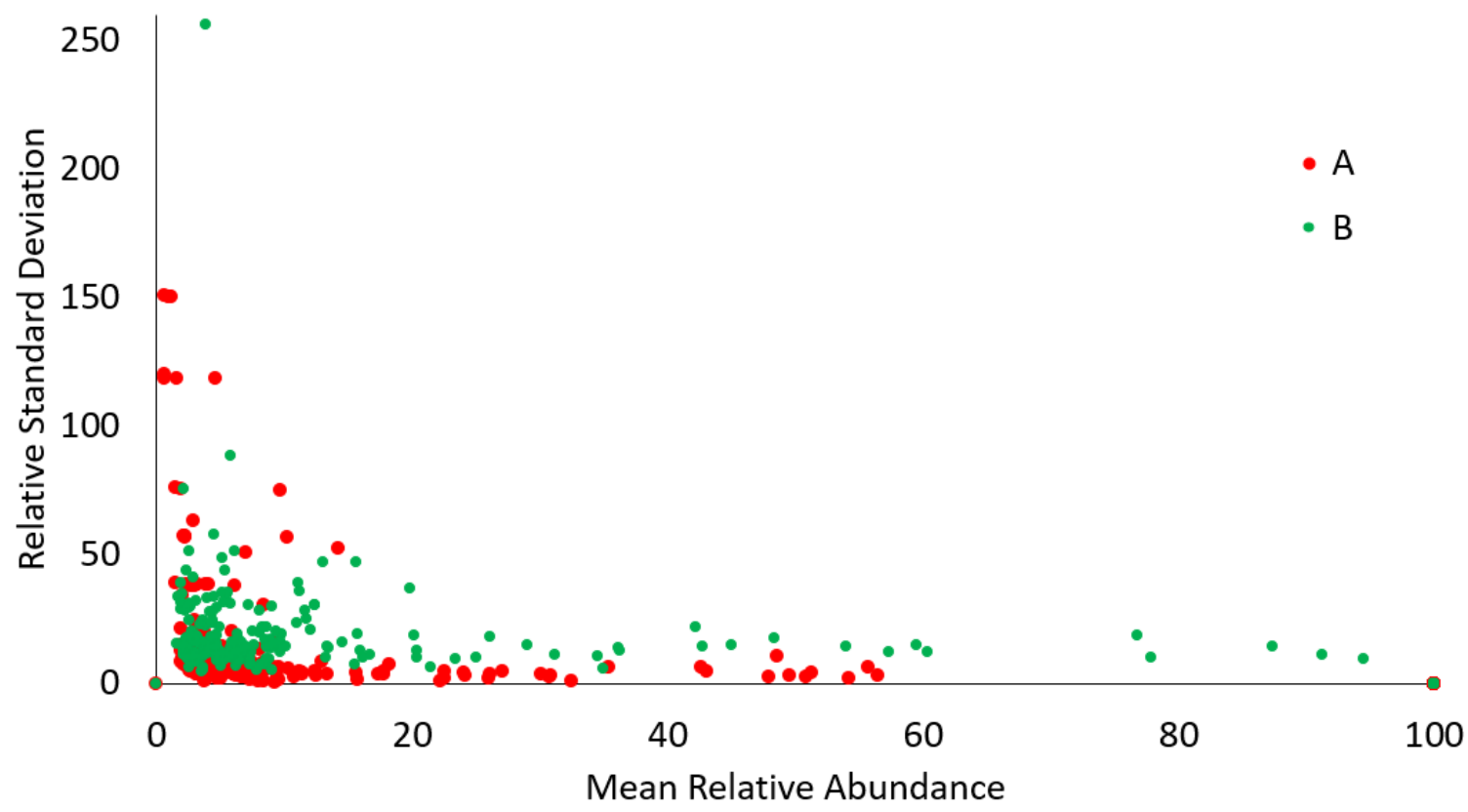

Figure A1b. \%RSD plotted as a function of mean \%RA for instruments A and B.

Data from instrument $\mathrm{C}$ was removed to highlight the exponential decay pattern elucidated. 


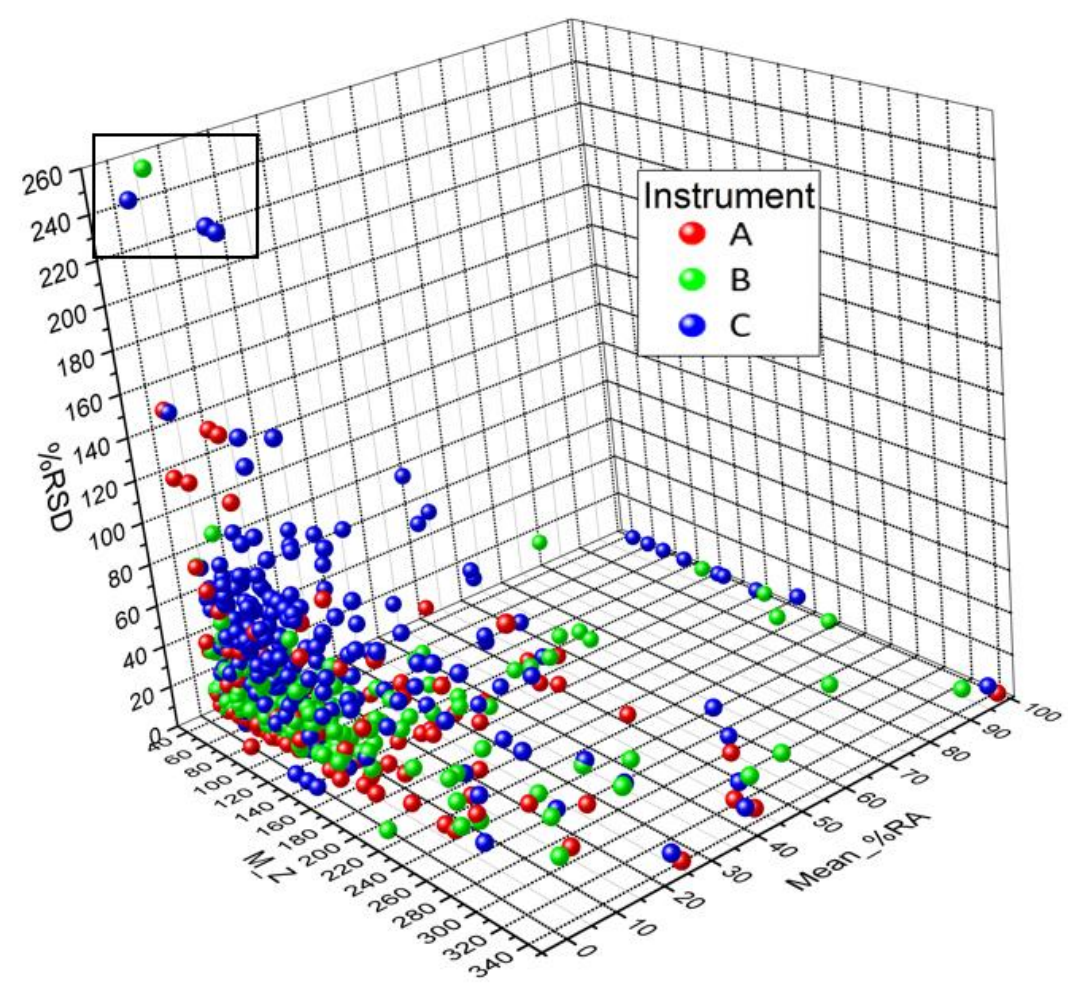

Figure A2. Three-dimensional plot comparing $\mathrm{m} / \mathrm{z}$, mean $\%$ RA and $\%$ RSD. Boxed data points are outliers which were investigated.

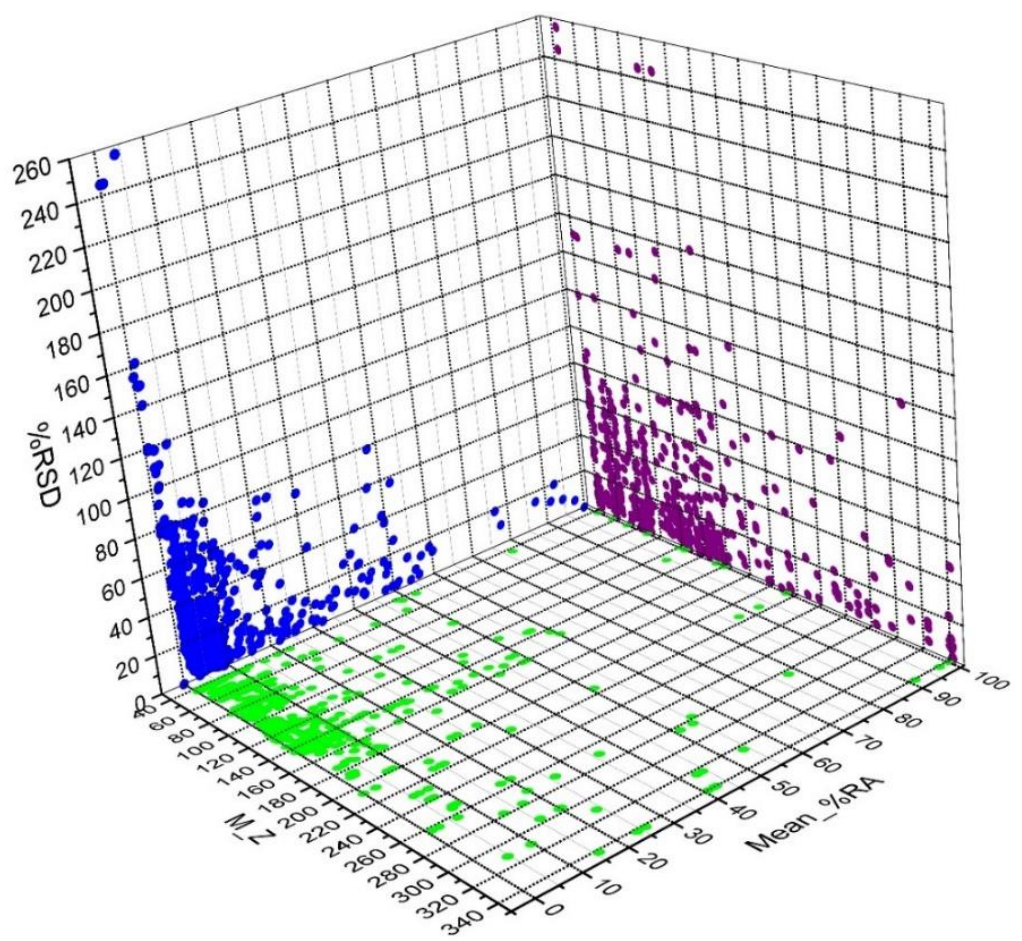

Figure A3. 3-dimensional wall projection. 
Table A1. Descriptive statistics for RIs for all NPSs.

The number of replicates for each system was: A $(n=9), B(n=14)$, and C $(n=6)$.

\begin{tabular}{|c|c|c|c|c|c|}
\hline System & Mean & Std. Dev. & $\%$ RSD & Upper $95 \%$ CI & Lower $95 \%$ CI \\
\hline \multicolumn{6}{|c|}{ 1-(3-chlorophenyl) piperazine } \\
\hline A & 1751.1 & 2.2 & 0.13 & 1749.4 & 1752.8 \\
\hline $\mathrm{B}$ & 1749.2 & 1.7 & 0.10 & 1748.3 & 1750.2 \\
\hline $\mathrm{C}$ & 1757.6 & 1.0 & 0.06 & 1756.5 & 1758.6 \\
\hline \multicolumn{6}{|c|}{ 2,5-dimethoxy-4-iodoamphetamine } \\
\hline A & 1939.4 & 1.3 & 0.07 & 1938.4 & 1940.4 \\
\hline B & 1922.0 & 1.9 & 0.10 & 1920.9 & 1923.1 \\
\hline $\mathrm{C}$ & 1944.2 & 1.1 & 0.05 & 1943.1 & 1945.3 \\
\hline \multicolumn{6}{|c|}{ 4-fluoroamphetamine } \\
\hline A & 1148.4 & 2.4 & 0.21 & 1146.6 & 1150.2 \\
\hline $\mathrm{B}$ & 1147.3 & 0.7 & 0.06 & 1146.9 & 1147.7 \\
\hline $\mathrm{C}$ & -- & -- & -- & -- & -- \\
\hline \multicolumn{6}{|c|}{ 25C-NBOMe } \\
\hline A & 2009.4 & 6.0 & 0.30 & 2004.9 & 2014.0 \\
\hline B & 1999.0 & 2.2 & 0.11 & 1997.8 & 2000.3 \\
\hline $\mathrm{C}$ & 2013.6 & 1.3 & 0.07 & 2012.2 & 2015.0 \\
\hline \multicolumn{6}{|c|}{ 25I-NBOMe } \\
\hline A & 2620.9 & 3.3 & 0.13 & 2618.4 & 2623.5 \\
\hline B & 2600.2 & 3.2 & 0.12 & 2598.3 & 2602.0 \\
\hline $\mathrm{C}$ & 2631.3 & 1.7 & 0.06 & 2629.6 & 2633.1 \\
\hline \multicolumn{6}{|c|}{ AH-7921 } \\
\hline A & 2828.9 & 3.0 & 0.10 & 2826.6 & 2831.2 \\
\hline $\mathrm{B}$ & 2805.7 & 3.2 & 0.12 & 2803.9 & 2807.6 \\
\hline $\mathrm{C}$ & 2842.7 & 1.7 & 0.06 & 2841.0 & 2844.5 \\
\hline \multicolumn{6}{|c|}{ Amphetamine } \\
\hline A & 2643.0 & 3.3 & 0.13 & 2640.5 & 2645.6 \\
\hline B & 2634.8 & 3.2 & 0.12 & 2633.0 & 2636.6 \\
\hline $\mathrm{C}$ & 2657.1 & 1.6 & 0.06 & 2655.5 & 2658.7 \\
\hline \multicolumn{6}{|c|}{ Benzyl piperazine } \\
\hline A & 1137.1 & 1.2 & 0.11 & 1136.2 & 1138.0 \\
\hline B & 1135.7 & 0.8 & 0.07 & 1135.2 & 1136.1 \\
\hline $\mathrm{C}$ & -- & -- & -- & -- & -- \\
\hline \multicolumn{6}{|c|}{ Butylone } \\
\hline A & 1502.5 & 4.9 & 0.33 & 1498.7 & 1506.2 \\
\hline $\mathrm{B}$ & 1498.8 & 1.1 & 0.07 & 1498.1 & 1499.4 \\
\hline $\mathrm{C}$ & 1503.0 & 1.5 & 0.10 & 1501.4 & 1504.6 \\
\hline \multicolumn{6}{|c|}{ N,N-Dimethyltryptamine } \\
\hline A & 1788.4 & 1.4 & 0.08 & 1787.3 & 1789.5 \\
\hline $\mathrm{B}$ & 1783.7 & 2.0 & 0.11 & 1782.6 & 1784.8 \\
\hline $\mathrm{C}$ & 1794.6 & 0.9 & 0.05 & 1793.6 & 1795.6 \\
\hline
\end{tabular}




\begin{tabular}{llllll}
\hline \multicolumn{5}{c}{ JWH-018 } \\
A & 1799.1 & 1.9 & 0.11 & 1797.7 & 1800.6 \\
B & 1794.7 & 1.9 & 0.11 & 1793.6 & 1795.8 \\
C & 1804.7 & 1.1 & 0.06 & 1803.6 & 1805.9 \\
\hline \multicolumn{5}{c}{ Mephedrone } \\
A & 3248.4 & 3.6 & 0.11 & 3245.7 & 3251.2 \\
B & 3233.4 & 5.5 & 0.17 & 3230.2 & 3236.6 \\
C & 3263.3 & 2.1 & 0.06 & 3261.1 & 3265.5 \\
\hline \multicolumn{7}{c}{ Methylone } \\
B & 1462.8 & 1.8 & 0.12 & 1461.4 & 1464.1 \\
C & 1460.5 & 1.3 & 0.09 & 1459.7 & 1461.2 \\
\hline A & 1466.4 & 1.1 & 0.08 & 1465.2 & 1467.5 \\
B & 1717.6 & 1.2 & 0.07 & 1716.7 & 1718.5 \\
C & 1712.0 & 2.5 & 0.15 & 1710.6 & 1713.5 \\
\hline
\end{tabular}

Table A2. Descriptive statistics for five most abundant ions for select NPSs. The number of replicates for each system was: A $(n=9), B(n=14)$, and C $(n=6)$.

\begin{tabular}{|c|c|c|c|c|c|c|}
\hline $\begin{array}{c}\text { Ion } \\
(m / z) \\
\end{array}$ & System & $\begin{array}{c}\text { Mean } \\
\text { RA } \\
\end{array}$ & $\begin{array}{l}\text { Std. } \\
\text { Dev. }\end{array}$ & \% RSD & $\begin{array}{c}\text { Lower } 95 \% \\
\text { CI } \\
\end{array}$ & $\begin{array}{c}\text { Upper } 95 \% \\
\text { CI }\end{array}$ \\
\hline \multicolumn{7}{|c|}{ 1-(3-chlorophenyl)piperazine } \\
\hline \multirow{3}{*}{156} & A & 32.5 & 0.3 & 0.76 & 32.3 & 32.7 \\
\hline & $\mathrm{B}$ & 35.0 & 2.1 & 5.96 & 33.8 & 36.2 \\
\hline & $\mathrm{C}$ & 32.6 & 3.1 & 9.35 & 29.4 & 35.8 \\
\hline \multirow{3}{*}{196} & $\mathrm{~A}$ & 22.2 & 0.2 & 0.78 & 22.1 & 22.3 \\
\hline & $\mathrm{B}$ & 23.4 & 2.3 & 9.67 & 22.1 & 24.7 \\
\hline & $\mathrm{C}$ & 27.9 & 2.6 & 9.32 & 25.2 & 30.7 \\
\hline \multirow{3}{*}{56} & $\mathrm{~A}$ & 8.7 & 0.5 & 6.24 & 8.3 & 9.1 \\
\hline & B & 13.3 & 1.3 & 10.05 & 12.5 & 14.1 \\
\hline & $\mathrm{C}$ & 46.7 & 28 & 58.96 & 17.8 & 75.5 \\
\hline \multirow{3}{*}{138} & A & 12.5 & 0.4 & 3.10 & 12.2 & 12.8 \\
\hline & B & 15.9 & 2.0 & 12.70 & 14.8 & 17.1 \\
\hline & $\mathrm{C}$ & 16.8 & 7.4 & 43.90 & 9.0 & 24.5 \\
\hline \multirow{3}{*}{111} & $\mathrm{~A}$ & 11.4 & 0.4 & 3.88 & 11.1 & 11.8 \\
\hline & B & 13.4 & 1.9 & 13.84 & 12.4 & 14.5 \\
\hline & $\mathrm{C}$ & 19.2 & 9.5 & 49.69 & 9.2 & 29.2 \\
\hline \multicolumn{7}{|c|}{ 2,5-dimethoxy-4-iodoamphetamine } \\
\hline \multirow{3}{*}{278} & $\mathrm{~A}$ & 55.7 & 3.6 & 6.53 & 52.9 & 58.5 \\
\hline & $\mathrm{B}$ & 16.7 & 1.8 & 10.98 & 15.7 & 17.8 \\
\hline & $\mathrm{C}$ & 12.5 & 5.7 & 45.53 & 6.5 & 18.5 \\
\hline \multirow{3}{*}{77} & $\mathrm{~A}$ & 9.2 & 0.6 & 6.54 & 8.7 & 9.6 \\
\hline & $\mathrm{B}$ & 5.2 & 0.6 & 11.50 & 4.8 & 5.5 \\
\hline & $\mathrm{C}$ & 3.4 & 0.8 & 21.85 & 2.6 & 4.2 \\
\hline
\end{tabular}




\begin{tabular}{|c|c|c|c|c|c|c|}
\hline \multirow{3}{*}{91} & A & 6.8 & 0.4 & 5.61 & 6.5 & 7.1 \\
\hline & $\mathrm{B}$ & 3.8 & 0.5 & 13.32 & 3.5 & 4.1 \\
\hline & $\mathrm{C}$ & 2.2 & 0.5 & 21.52 & 1.7 & 2.7 \\
\hline \multirow{3}{*}{263} & A & 6.4 & 0.4 & 5.64 & 6.2 & 6.7 \\
\hline & $\mathrm{B}$ & 2.4 & 0.4 & 17.70 & 2.2 & 2.7 \\
\hline & $\mathrm{C}$ & 0.9 & 0.8 & 91.84 & 0.0 & 1.8 \\
\hline \multirow{3}{*}{42} & A & 3.3 & 0.2 & 7.11 & 3.1 & 3.5 \\
\hline & B & 2.7 & 0.4 & 15.91 & 2.4 & 2.9 \\
\hline & $\mathrm{C}$ & 3.8 & 2.4 & 63.16 & 1.3 & 6.4 \\
\hline \multicolumn{7}{|c|}{ 25C-NBOMe } \\
\hline \multirow{3}{*}{150} & A & 35.4 & 2.2 & 6.16 & 33.7 & 37.0 \\
\hline & $\mathrm{B}$ & 42.7 & 6.1 & 14.37 & 39.2 & 46.3 \\
\hline & $\mathrm{C}$ & 39.2 & 3.8 & 9.76 & 35.2 & 43.2 \\
\hline \multirow{3}{*}{91} & $\mathrm{~A}$ & 30.9 & 1.0 & 3.17 & 30.2 & 31.7 \\
\hline & $\mathrm{B}$ & 34.6 & 3.8 & 10.84 & 32.4 & 36.7 \\
\hline & $\mathrm{C}$ & 49.2 & 21 & 41.89 & 27.6 & 70.8 \\
\hline \multirow{3}{*}{122} & $\mathrm{~A}$ & 9.4 & 0.2 & 2.07 & 9.3 & 9.6 \\
\hline & $\mathrm{B}$ & 9.1 & 0.5 & 5.36 & 8.8 & 9.4 \\
\hline & $\mathrm{C}$ & 9.6 & 0.9 & 8.85 & 8.7 & 10.5 \\
\hline \multirow{3}{*}{77} & $\mathrm{~A}$ & 6.4 & 0.8 & 12.94 & 5.8 & 7.0 \\
\hline & $\mathrm{B}$ & 6.9 & 0.9 & 12.43 & 6.4 & 7.4 \\
\hline & $\mathrm{C}$ & 11.0 & 6.5 & 58.99 & 4.2 & 17.8 \\
\hline \multirow{3}{*}{65} & $\mathrm{~A}$ & 4.1 & 1.6 & 38.63 & 2.9 & 5.3 \\
\hline & $\mathrm{B}$ & 6.3 & 0.8 & 12.21 & 5.8 & 6.7 \\
\hline & $\mathrm{C}$ & 12.0 & 6.7 & 56.19 & 4.9 & 19.1 \\
\hline \multicolumn{7}{|c|}{ "Amphetamine } \\
\hline \multirow{3}{*}{91} & A & 24.0 & 1.0 & 4.07 & 23.3 & 24.8 \\
\hline & $\mathrm{B}$ & 15.6 & 1.2 & 7.62 & 14.9 & 16.2 \\
\hline & $\mathrm{C}$ & 9.5 & 3.3 & 34.67 & 6.0 & 12.9 \\
\hline \multirow{3}{*}{65} & $\mathrm{~A}$ & 12.4 & 0.6 & 4.60 & 11.9 & 12.8 \\
\hline & $\mathrm{B}$ & 7.9 & 0.4 & 5.17 & 7.6 & 8.1 \\
\hline & $\mathrm{C}$ & 7.3 & 3.1 & 42.67 & 4.0 & 10.5 \\
\hline \multirow{3}{*}{42} & $\mathrm{~A}$ & 6.0 & 0.3 & 4.35 & 5.8 & 6.2 \\
\hline & B & 5.1 & 0.4 & 7.08 & 4.9 & 5.3 \\
\hline & $\mathrm{C}$ & 6.9 & 4.1 & 58.78 & 2.7 & 11.2 \\
\hline \multirow{3}{*}{51} & $\mathrm{~A}$ & 4.8 & 0.3 & 5.60 & 4.6 & 5.0 \\
\hline & B & 3.2 & 0.3 & 8.39 & 3.1 & 3.4 \\
\hline & $\mathrm{C}$ & 3.6 & 1.8 & 49.14 & 1.7 & 5.5 \\
\hline \multirow{3}{*}{63} & $\mathrm{~A}$ & 4.5 & 0.3 & 6.35 & 4.3 & 4.8 \\
\hline & B & 3.1 & 0.4 & 11.92 & 2.9 & 3.3 \\
\hline & $\mathrm{C}$ & 2.8 & 1.8 & 64.55 & 0.9 & 4.8 \\
\hline
\end{tabular}




\begin{tabular}{|c|c|c|c|c|c|c|}
\hline \multicolumn{7}{|c|}{ JWH-018 } \\
\hline \multirow{3}{*}{341} & A & 100.0 & 0.0 & 0.00 & 100.0 & 100.0 \\
\hline & $\mathrm{B}$ & 91.3 & 10 & 10.99 & 85.5 & 97.1 \\
\hline & $\mathrm{C}$ & 97.7 & 5.7 & 5.79 & 91.8 & 103.6 \\
\hline \multirow{3}{*}{284} & A & 54.3 & 1.1 & 2.03 & 53.4 & 55.1 \\
\hline & $\mathrm{B}$ & 77.9 & 7.9 & 10.18 & 73.3 & 82.5 \\
\hline & $\mathrm{C}$ & 51.2 & 14 & 26.71 & 36.9 & 65.6 \\
\hline \multirow{3}{*}{214} & $\mathrm{~A}$ & 50.8 & 1.4 & 2.76 & 49.7 & 51.9 \\
\hline & $\mathrm{B}$ & 87.4 & 13 & 14.43 & 80.1 & 94.6 \\
\hline & $\mathrm{C}$ & 31.1 & 13 & 41.79 & 17.5 & 44.8 \\
\hline \multirow{3}{*}{127} & A & 51.2 & 2.1 & 4.01 & 49.7 & 52.8 \\
\hline & $\mathrm{B}$ & 94.5 & 8.8 & 9.30 & 89.5 & 99.6 \\
\hline & $\mathrm{C}$ & 42.5 & 9.5 & 22.27 & 32.6 & 52.4 \\
\hline \multirow{3}{*}{324} & A & 43.0 & 2.1 & 4.92 & 41.4 & 44.7 \\
\hline & $\mathrm{B}$ & 54.0 & 7.9 & 14.60 & 49.5 & 58.6 \\
\hline & $\mathrm{C}$ & 44.3 & 5.2 & 11.66 & 38.9 & 49.8 \\
\hline \multicolumn{7}{|c|}{ 5-MeO-AMT } \\
\hline \multirow{3}{*}{161} & A & 100.0 & 0.0 & 0.00 & 100.0 & 100.0 \\
\hline & $\mathrm{B}$ & 100.0 & 0.0 & 0.00 & 100.0 & 100.0 \\
\hline & $\mathrm{C}$ & 42.5 & 18 & 41.19 & 24.1 & 60.9 \\
\hline \multirow{3}{*}{44} & $\mathrm{~A}$ & 48.6 & 5.3 & 10.83 & 44.5 & 52.6 \\
\hline & $\mathrm{B}$ & 76.8 & 14 & 18.73 & 68.5 & 85.1 \\
\hline & $\mathrm{C}$ & 100.0 & 0.0 & 0.00 & 100.0 & 100.0 \\
\hline \multirow{3}{*}{160} & $\mathrm{~A}$ & 49.6 & 1.6 & 3.13 & 48.4 & 50.8 \\
\hline & B & 48.3 & 8.6 & 17.84 & 43.4 & 53.3 \\
\hline & $\mathrm{C}$ & 19.1 & 6.4 & 33.27 & 12.4 & 25.8 \\
\hline \multirow{3}{*}{146} & $\mathrm{~A}$ & 24.2 & 0.7 & 2.86 & 23.6 & 24.7 \\
\hline & $\mathrm{B}$ & 25.0 & 2.5 & 10.16 & 23.5 & 26.5 \\
\hline & $\mathrm{C}$ & 9.6 & 4.2 & 43.78 & 5.2 & 14.0 \\
\hline \multirow{3}{*}{117} & $\mathrm{~A}$ & 17.8 & 0.9 & 4.80 & 17.2 & 18.5 \\
\hline & $\mathrm{B}$ & 20.2 & 3.8 & 18.82 & 18.0 & 22.4 \\
\hline & $\mathrm{C}$ & 12.2 & 3.8 & 31.38 & 8.2 & 16.3 \\
\hline \multicolumn{7}{|c|}{ UR-144 } \\
\hline \multirow{3}{*}{214} & A & 100.0 & 0.0 & 0.00 & 100.0 & 100.0 \\
\hline & $\mathrm{B}$ & 100.0 & 0.0 & 0.00 & 100.0 & 100.0 \\
\hline & $\mathrm{C}$ & 92.6 & 18 & 19.56 & 73.6 & 111.6 \\
\hline \multirow{3}{*}{144} & $\mathrm{~A}$ & 22.6 & 0.5 & 1.97 & 22.2 & 22.9 \\
\hline & B & 31.2 & 3.5 & 11.22 & 29.2 & 33.2 \\
\hline & $\mathrm{C}$ & 24.4 & 3.4 & 14.04 & 20.8 & 28.0 \\
\hline \multirow{3}{*}{43} & $\mathrm{~A}$ & 7.4 & 0.6 & 8.25 & 6.9 & 7.9 \\
\hline & B & 15.7 & 31 & 19.44 & 13.9 & 17.5 \\
\hline & $\mathrm{C}$ & 52.2 & 30 & 56.84 & 21.1 & 83.3 \\
\hline
\end{tabular}




\begin{tabular}{ccccccc}
\hline \multirow{3}{*}{296} & A & 15.7 & 0.7 & 4.33 & 15.2 & 16.2 \\
& B & 19.9 & 7.4 & 37.12 & 15.6 & 24.1 \\
& C & 20.3 & 7.9 & 38.80 & 12.0 & 28.6 \\
\hline \multirow{3}{*}{41} & A & 6.1 & 0.6 & 9.47 & 5.6 & 6.5 \\
& B & 12.4 & 3.8 & 30.76 & 10.2 & 14.6 \\
& C & 47.9 & 37 & 78.35 & 8.5 & 87.2 \\
\hline
\end{tabular}

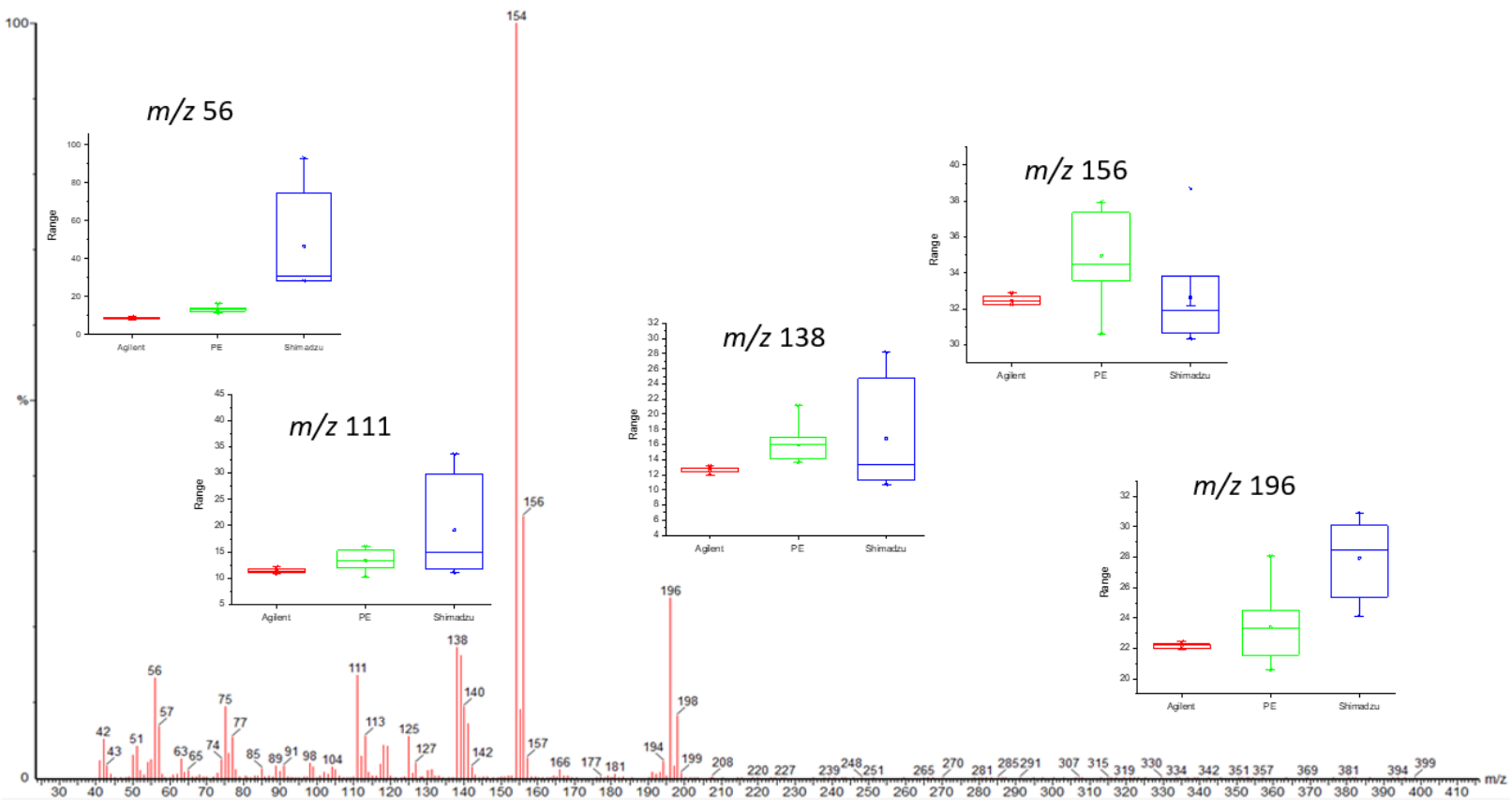

Figure A4. 1-(3-chlorophenyl)piperazine mass spectra with box comparison charts of the top five ions. 


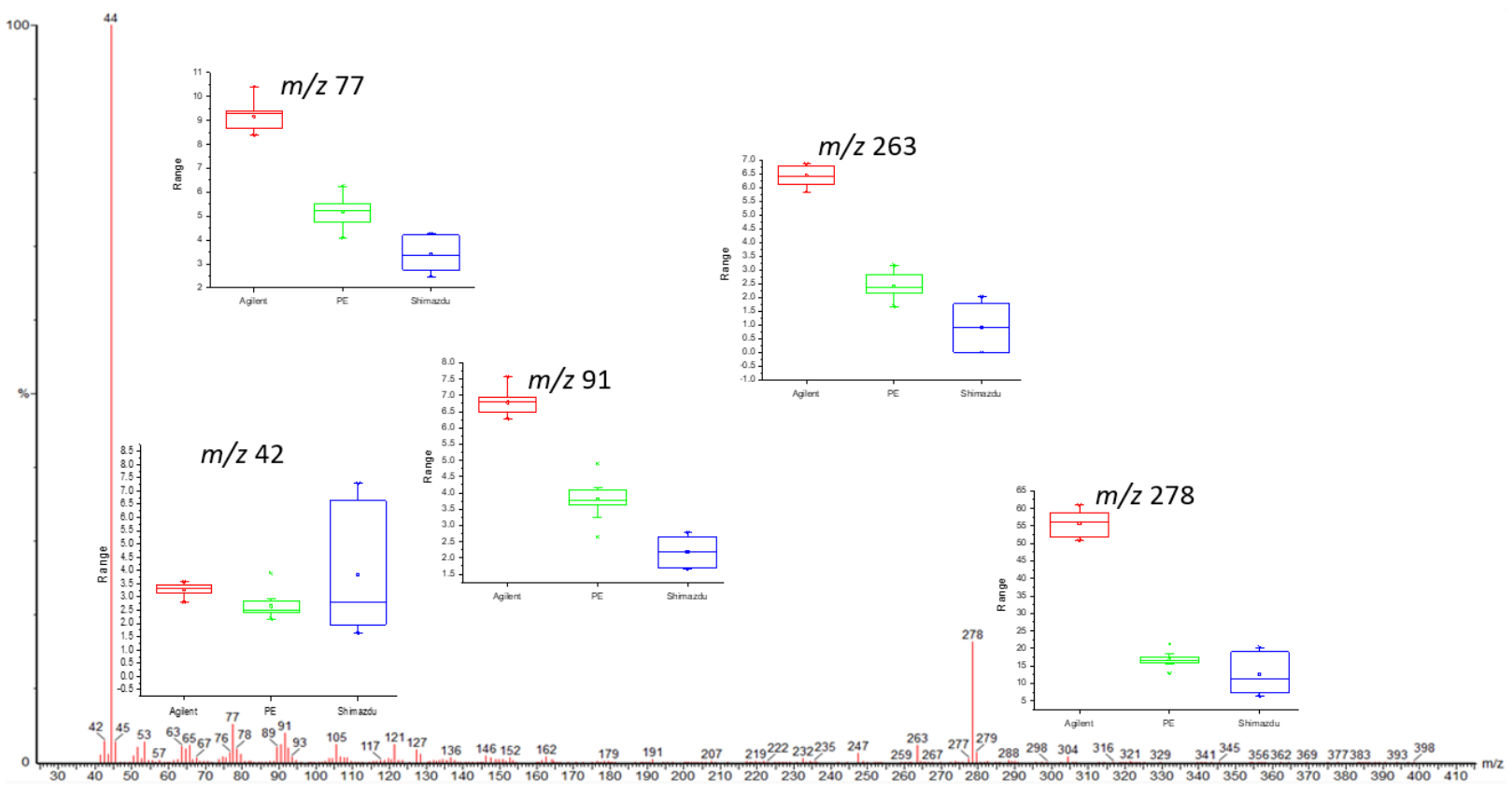

Figure A5. 2,5-dimethoxy-4-iodoamphetamine mass spectra with box comparison charts of the top five ions.

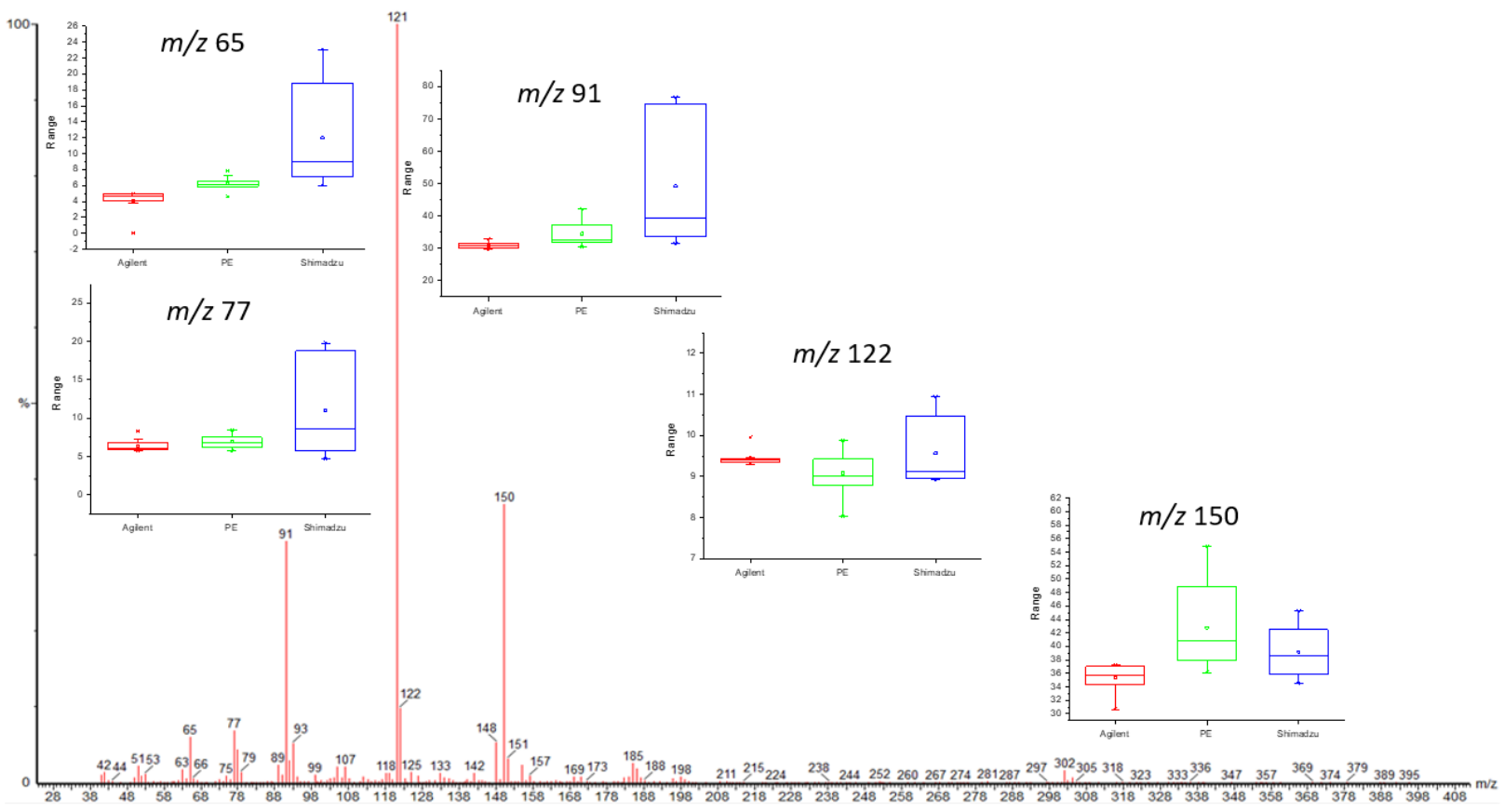

Figure A6. 25C-NBOMe mass spectra with box comparison charts of the top five ions. 


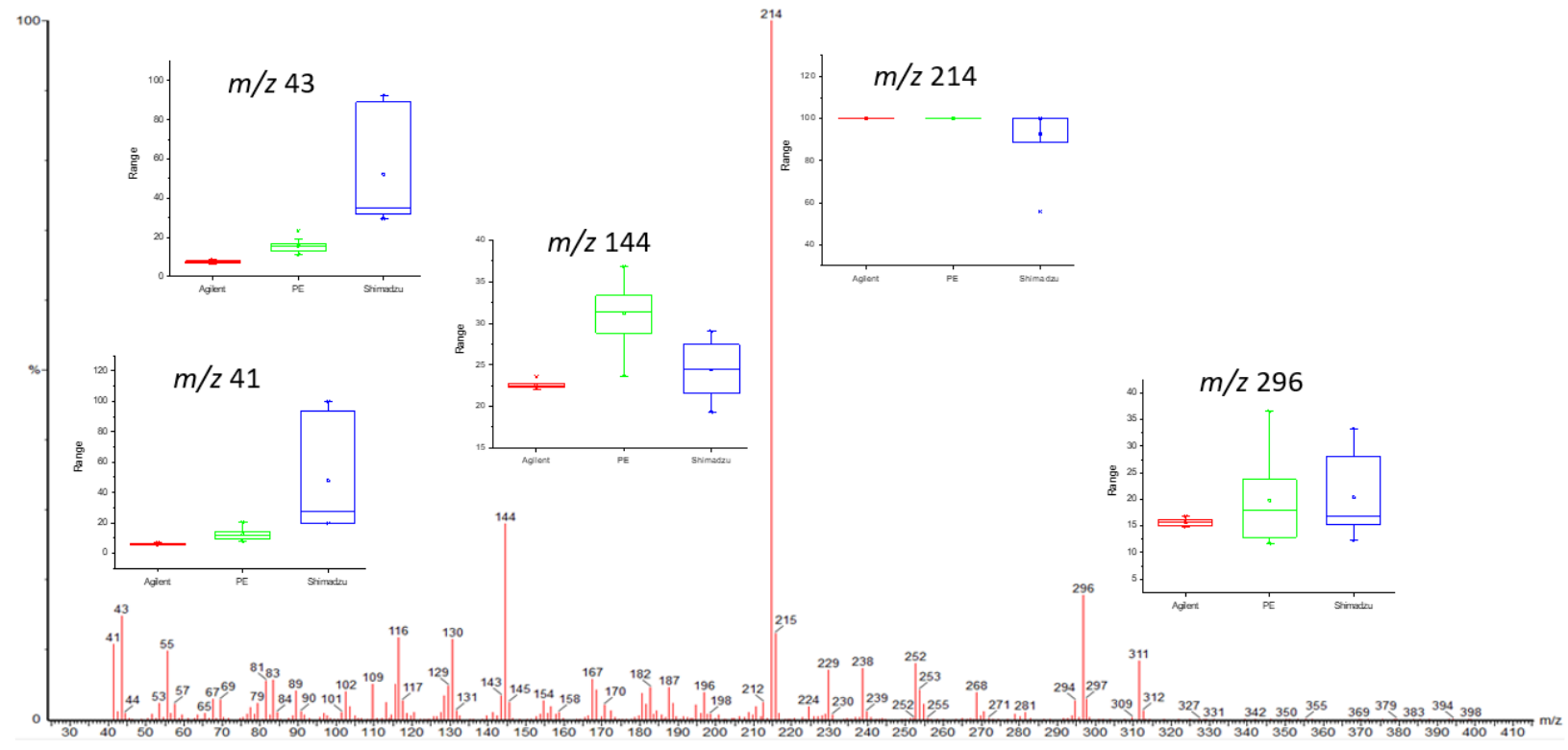

Figure A7. UR-144 mass spectra with box comparison charts for top five most abundant ions.

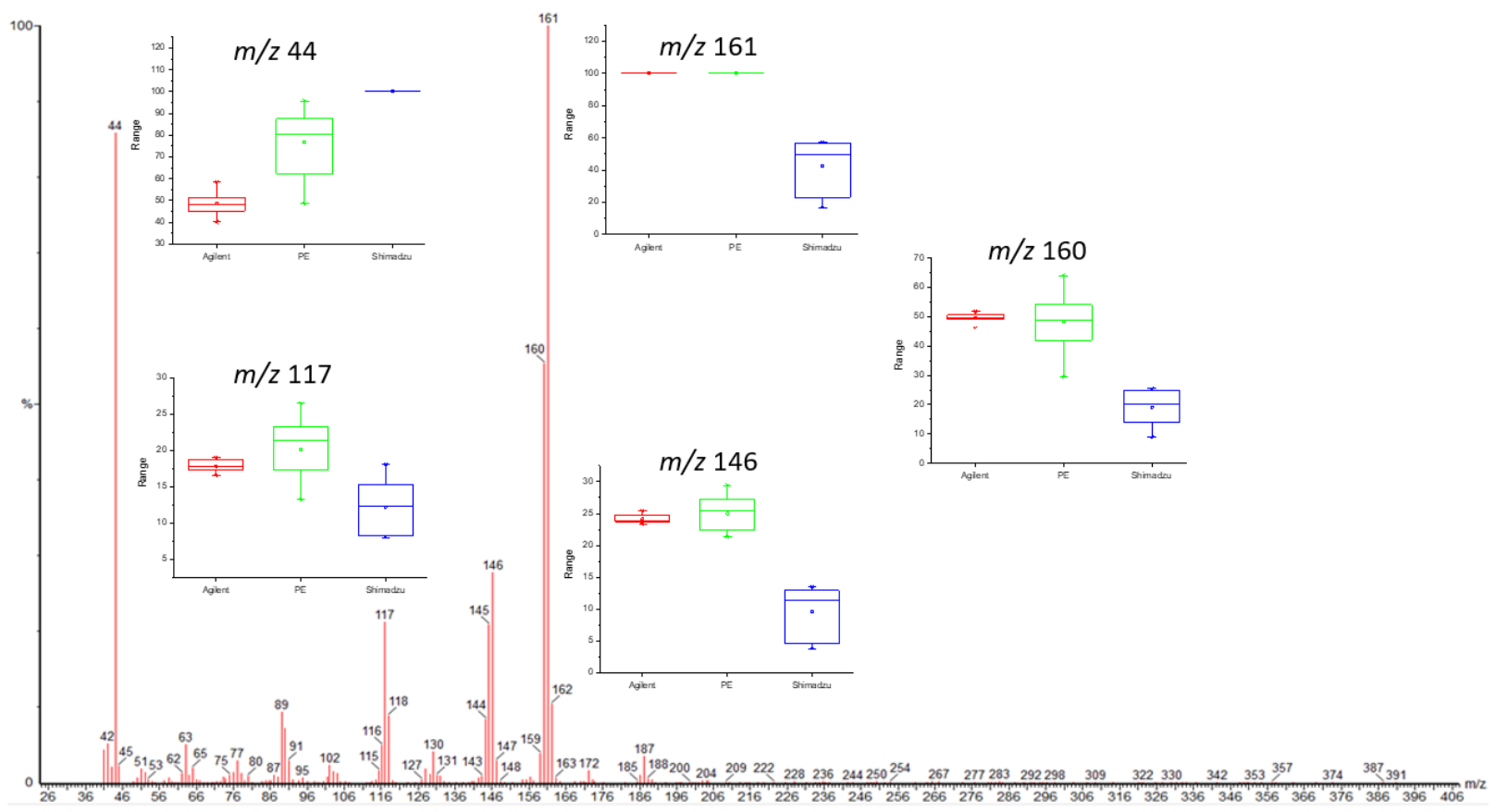

Figure A8. 5-MeO-AMT mass spectra with box comparison charts for top five most abundant ions. 


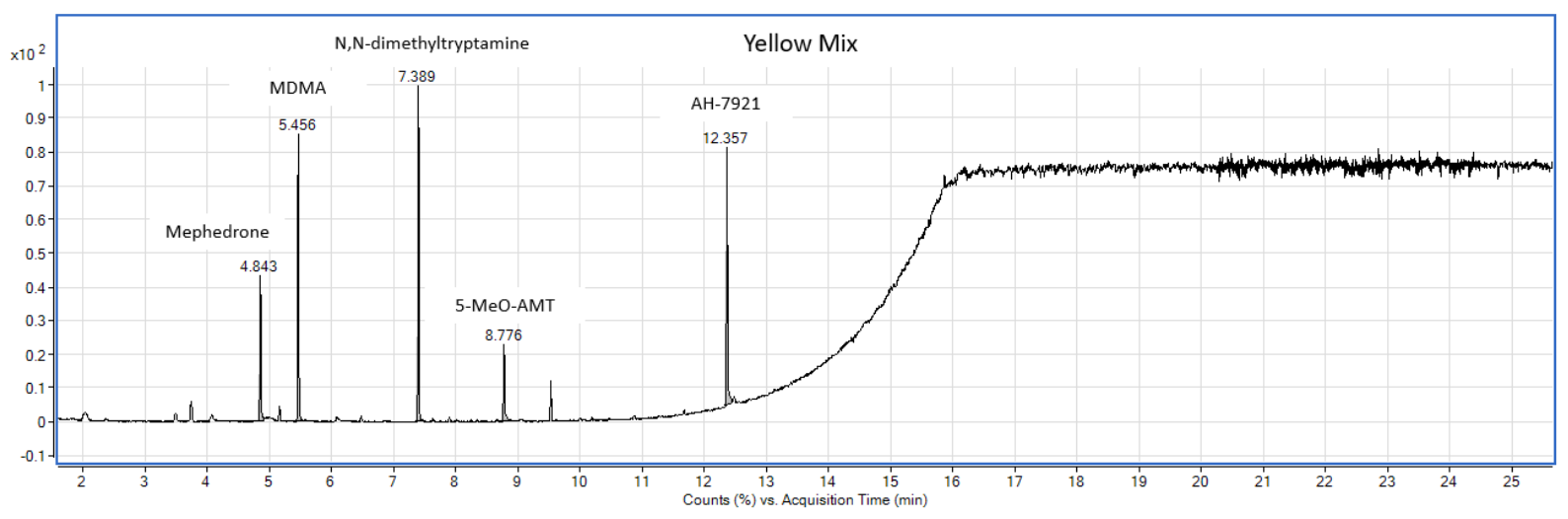

Figure A9a. Chromatogram from Yellow mix.

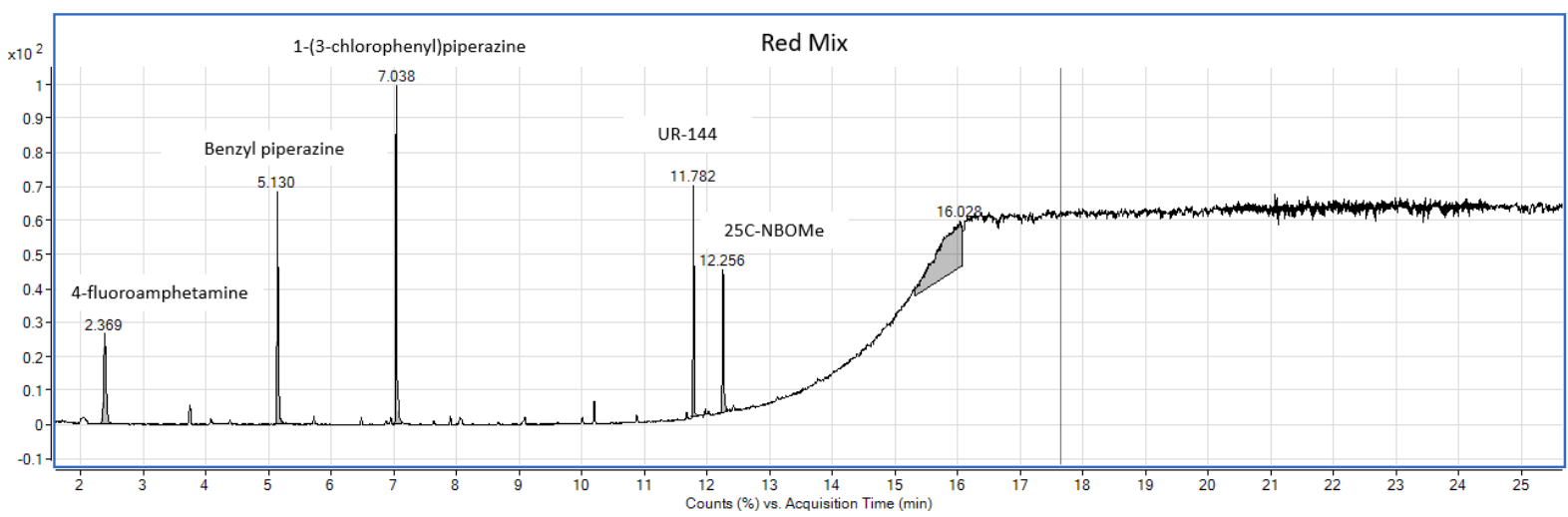

Figure A9b. Chromatogram from Red mix.

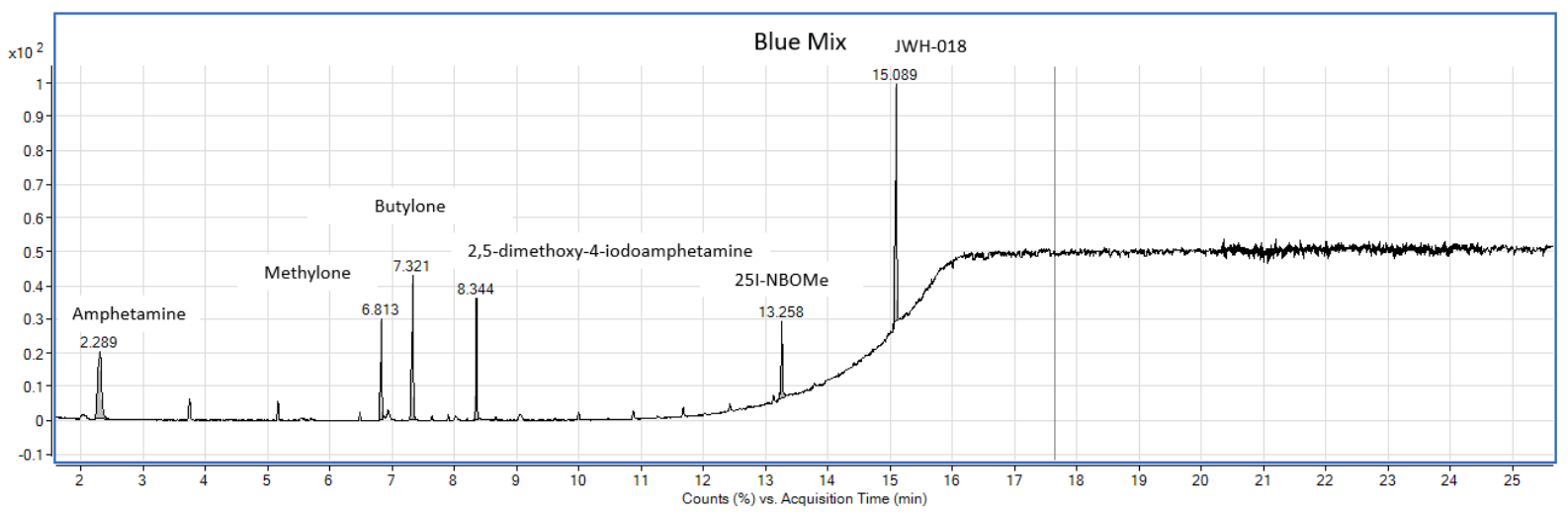

Figure A9c. Chromatogram from Blue mix.

The overall signal is low ( $10^{2}$ on all instruments), which is expected as the concentrations of individual compounds chosen was low. The overall width for 4-fluoroamphetamine and amphetamine was consistently wider than all other compounds; it is postulated that this widening was due to the compounds eluting near the solvent. Furthermore, these were the only peaks which showed a slight amount of tailing. All other compounds showed narrow and symmetrical 
peaks. The compound concentrations were optimized so that the chromatogram peak was $\sim 30 \%$ normalized; however, some peak heights varied and would occasionally be present below this threshold (see 5-MeO-AMT in yellow mix). 


\section{Appendix B.}

\section{Chapter 3: The Effect of Mass Spectrometry Tuning Frequency and Criteria on Ion Relative Abundances of Cathinones and Cannabinoids - Supplemental Information}

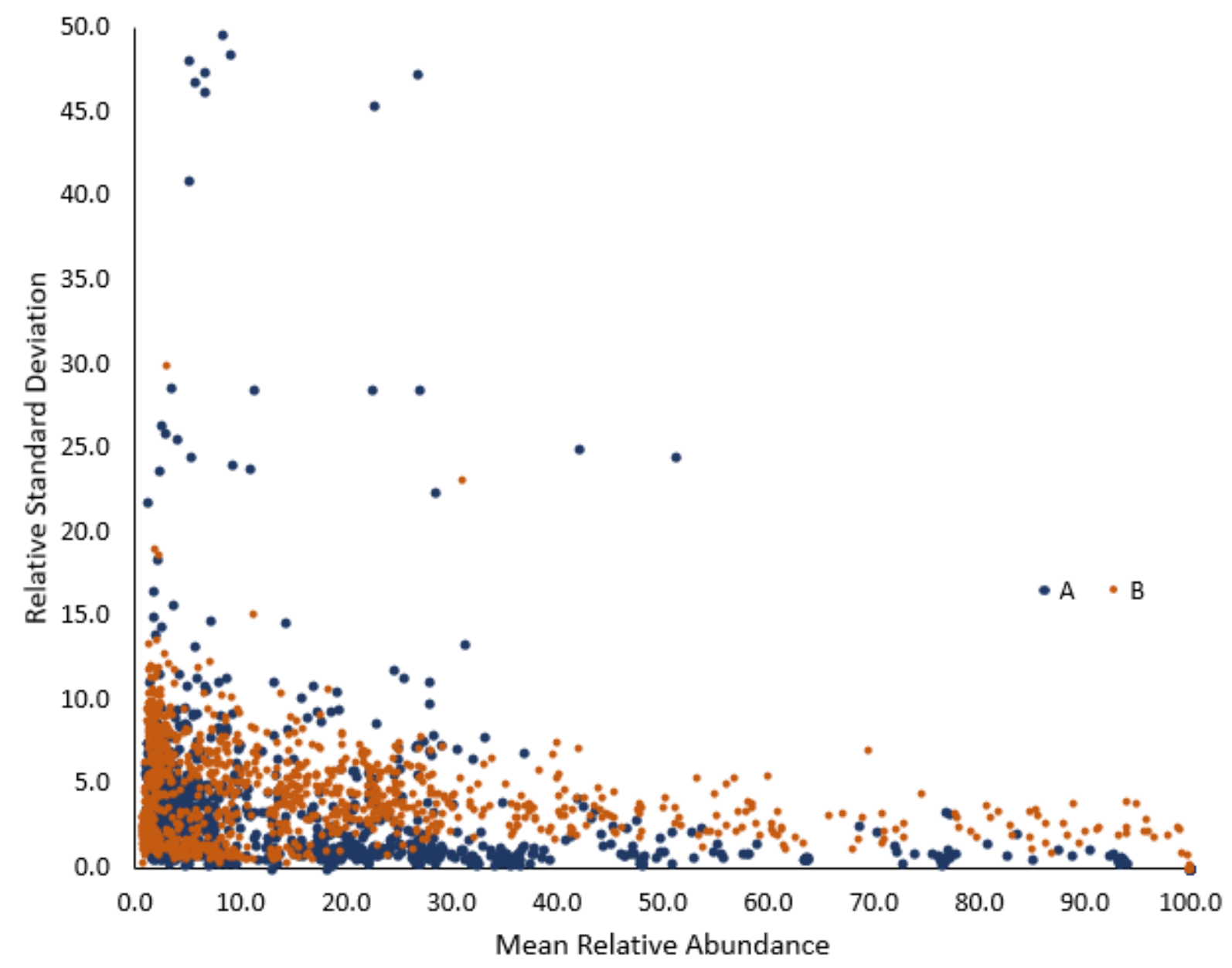

Figure B1. \% RSD plotted as a function of the mean RA from single day analysis sets from all NPSs and separated by instruments.

( $n=3$ to 10 depends on the individual data point, i.e. the ion was recorded a minimum of 3 times in a single day). Instrument A markers are enlarged by $0.25 \mathrm{pts}$ to increase visibility of data points located behind instrument $B$ data points. 


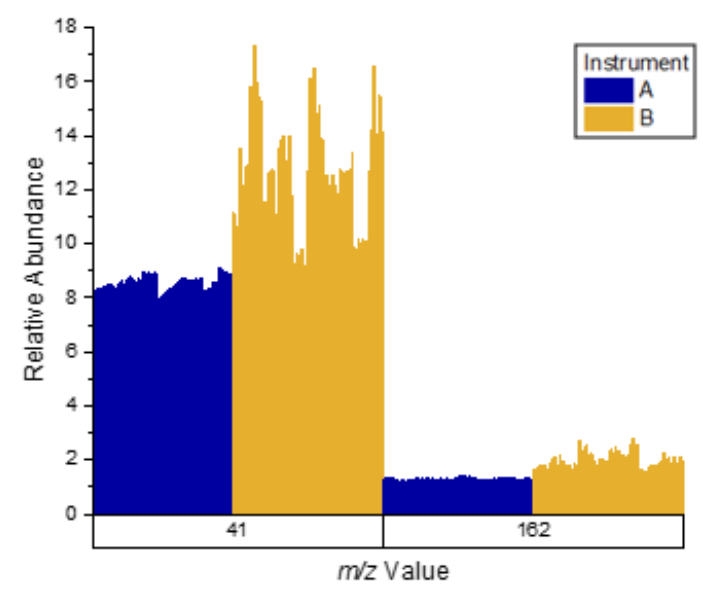

Figure B2a. All recorded RAs for select ions from NEB.

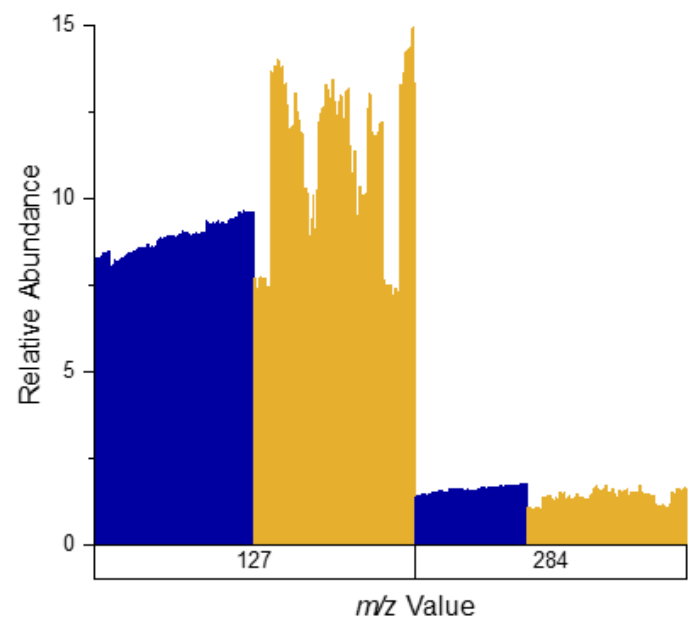

Figure B2b. All recorded RAs for select ions from JWH-200.

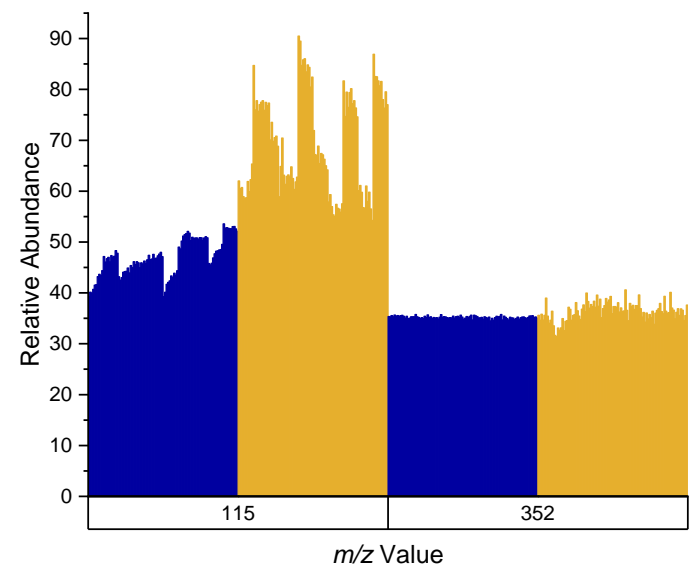

Figure B2c. All recorded RAs for select ions from JWH-149.

Note y-axis scale change. 


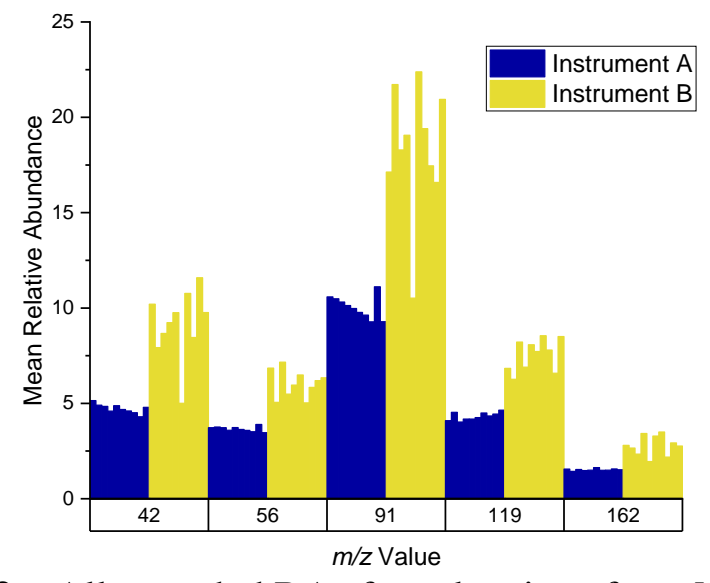

Figure B3a. All recorded RAs for select ions from MeMABP.

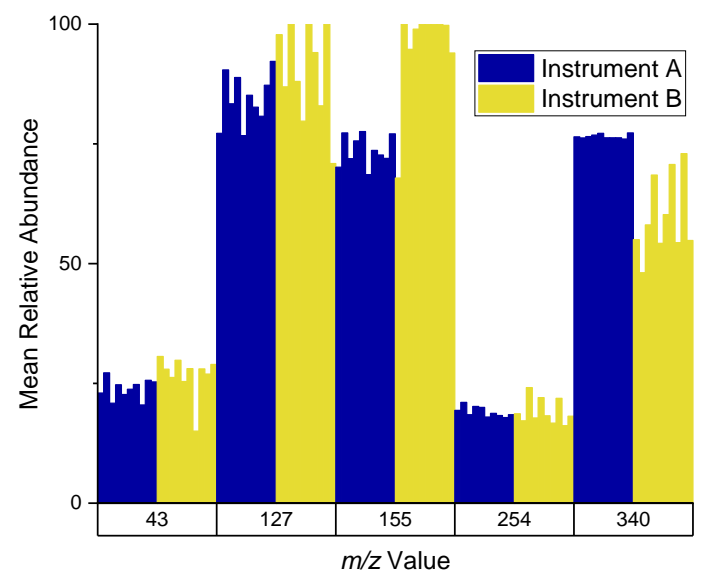

Figure B3b. All recorded RAs for select ions from JWH-116.

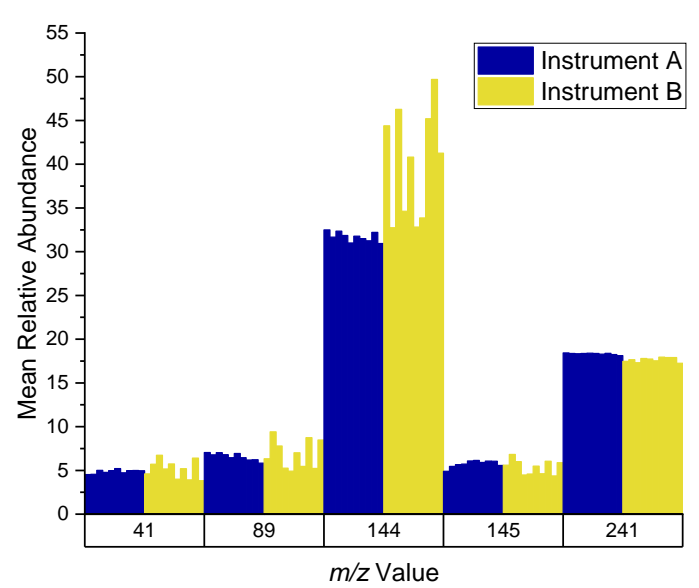

Figure B3c. All recorded RAs for select ions from BB-22.

Note $y$-axis scale change. $n=3$ to 10 depending how many times the ion was recorded each day. 

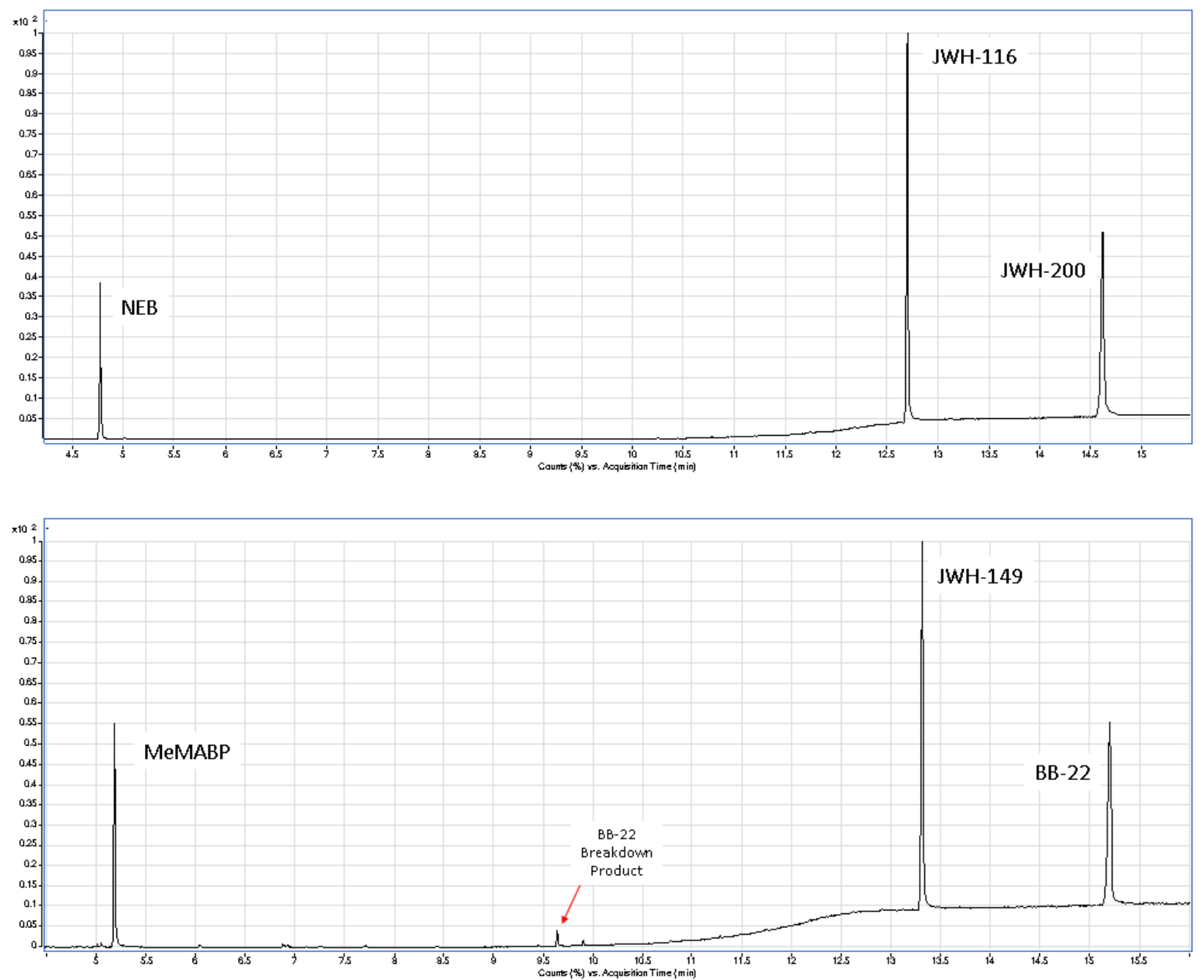

Figure B4. Sample chromatogram for NPS solutions. 


\section{Appendix C.}

\section{Chapter 4: Synthetic Small Molecule Characterization and Isomer Discrimination Using Gas-phase Hydrogen Deuterium Exchange IMS-MS - Supplemental Information}

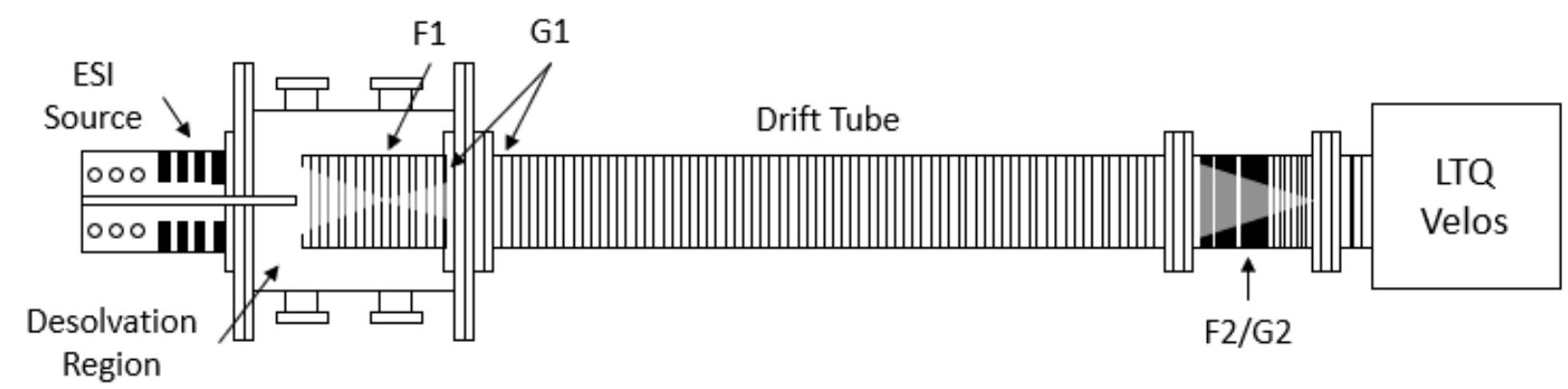

Figure C1. Schematic of the home-build drift tube used.

The desolvation region contains funnel 1 (F1) and gate 1 (G1) where the ions are focused and stored before entering the drift tube. Funnel 2 (F2) focuses the ion beams and the gating pulse at gate $2(\mathrm{G} 2)$ is used to filter ions based on drift time.

Table C1. Experimental parameters used for CCS calculations.

Thick horizontal line separates the initial evaluation of HDX and the compounds used for isomer discrimination investigation. Additional parameters (abbreviations correspond to those defined in equation 1): $T=296 \mathrm{~K}, E=800 \mathrm{~V}$, and $m_{b}=4 \mathrm{~g} / \mathrm{mol}$.

\begin{tabular}{|c|c|c|c|c|c|}
\hline Compound & Structure & $\begin{array}{l}\text { M.W. } \\
\text { (g/mol) }\end{array}$ & Parameter & Trial 1 & Trial 2 \\
\hline 4-fluoroamphetamine & & 154 & $\begin{array}{c}\mathrm{P} \text { (torr) } \\
\mathrm{L}(\mathrm{m}) \\
\mathrm{t}_{\mathrm{D}}(\mathrm{ms})\end{array}$ & $\begin{array}{l}2.63 \\
0.99 \\
4.95\end{array}$ & $\begin{array}{l}2.54 \\
0.93 \\
4.36\end{array}$ \\
\hline Benzyl piperazine & & 177 & $\begin{array}{c}\mathrm{P}(\text { torr }) \\
\mathrm{L}(\mathrm{m}) \\
\mathrm{t}_{\mathrm{D}}(\mathrm{ms})\end{array}$ & $\begin{array}{l}2.70 \\
0.99 \\
5.60 \\
\end{array}$ & $\begin{array}{l}2.54 \\
0.93 \\
4.80 \\
\end{array}$ \\
\hline 5-MeO-AMT & & 205 & $\begin{array}{c}\mathrm{P} \text { (torr) } \\
\mathrm{L}(\mathrm{m}) \\
\mathrm{t}_{\mathrm{D}}(\mathrm{ms})\end{array}$ & $\begin{array}{l}2.69 \\
0.99 \\
6.06\end{array}$ & $\begin{array}{l}2.57 \\
0.93 \\
5.28\end{array}$ \\
\hline Methylone & & 208 & $\begin{array}{c}\mathrm{P} \text { (torr) } \\
\mathrm{L}(\mathrm{m}) \\
\mathrm{t}_{\mathrm{D}}(\mathrm{ms})\end{array}$ & $\begin{array}{l}2.67 \\
0.99 \\
5.80\end{array}$ & $\begin{array}{l}2.57 \\
0.93 \\
5.03\end{array}$ \\
\hline АН-7921 & & 329 & $\begin{array}{c}\mathrm{P} \text { (torr) } \\
\mathrm{L}(\mathrm{m}) \\
\mathrm{t}_{\mathrm{D}}(\mathrm{ms})\end{array}$ & $\begin{array}{l}2.66 \\
0.99 \\
7.79\end{array}$ & $\begin{array}{l}2.57 \\
0.93 \\
6.84\end{array}$ \\
\hline
\end{tabular}




\begin{tabular}{|c|c|c|c|c|}
\hline 25C-NBOMe & 336 & $\begin{array}{c}\mathrm{P} \text { (torr) } \\
\mathrm{L}(\mathrm{m}) \\
\mathrm{t}_{\mathrm{D}}(\mathrm{ms})\end{array}$ & $\begin{array}{l}2.58 \\
0.99 \\
8.02\end{array}$ & $\begin{array}{l}2.57 \\
0.93 \\
7.05\end{array}$ \\
\hline Ortho-* & 181 & $\begin{array}{c}\mathrm{P}(\text { torr }) \\
\mathrm{L}(\mathrm{m}) \\
\mathrm{t}_{\mathrm{D}}(\mathrm{ms})\end{array}$ & $\begin{array}{l}2.54 \\
0.93 \\
4.77\end{array}$ & $\begin{array}{l}2.54 \\
0.93 \\
4.85\end{array}$ \\
\hline Meta-* & 181 & $\begin{array}{c}\mathrm{P} \text { (torr) } \\
\mathrm{L}(\mathrm{m}) \\
\mathrm{t}_{\mathrm{D}}(\mathrm{ms})\end{array}$ & $\begin{array}{l}2.54 \\
0.93 \\
4.90\end{array}$ & $\begin{array}{l}2.54 \\
0.93 \\
4.88\end{array}$ \\
\hline Para-* & 181 & $\begin{array}{c}\mathrm{P}(\text { torr }) \\
\mathrm{L}(\mathrm{m}) \\
\mathrm{t}_{\mathrm{D}}(\mathrm{ms})\end{array}$ & $\begin{array}{l}2.54 \\
0.93 \\
4.80 \\
\end{array}$ & $\begin{array}{l}2.54 \\
0.93 \\
4.89 \\
\end{array}$ \\
\hline
\end{tabular}

*Refers to fluorophenyl piperazine isomers

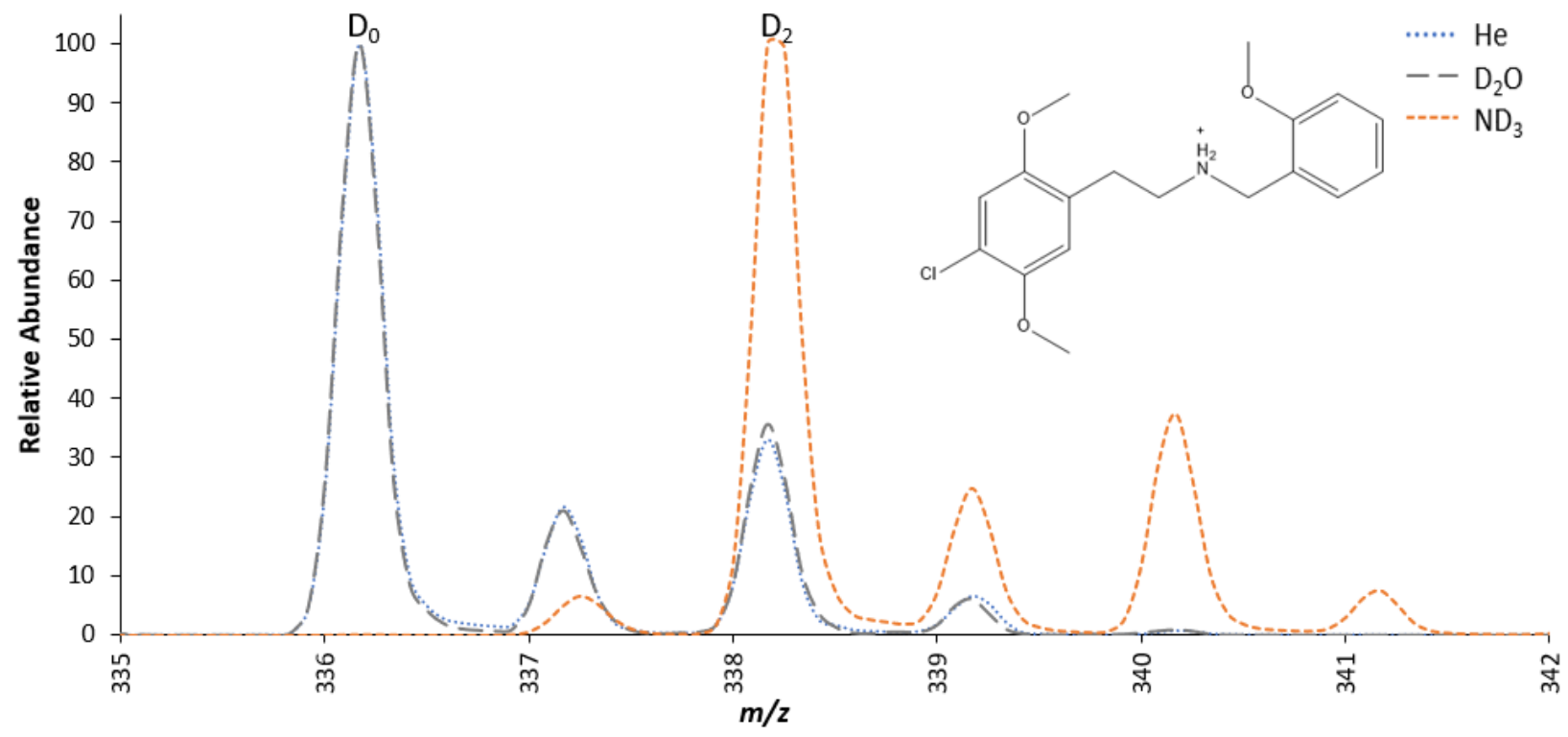

Figure C2a. Isotopic distribution for 25C-NBOMe.

No deuterium uptake occurs in the presence of $\mathrm{D}_{2} \mathrm{O}$. In the presence of $\mathrm{ND}_{3}$, ions exchanged 2 hydrogens; the peak at $\mathrm{m} / \mathrm{z} 340$ is the chlorine isotopic peak and is not the result of additional deuterium exchange. 


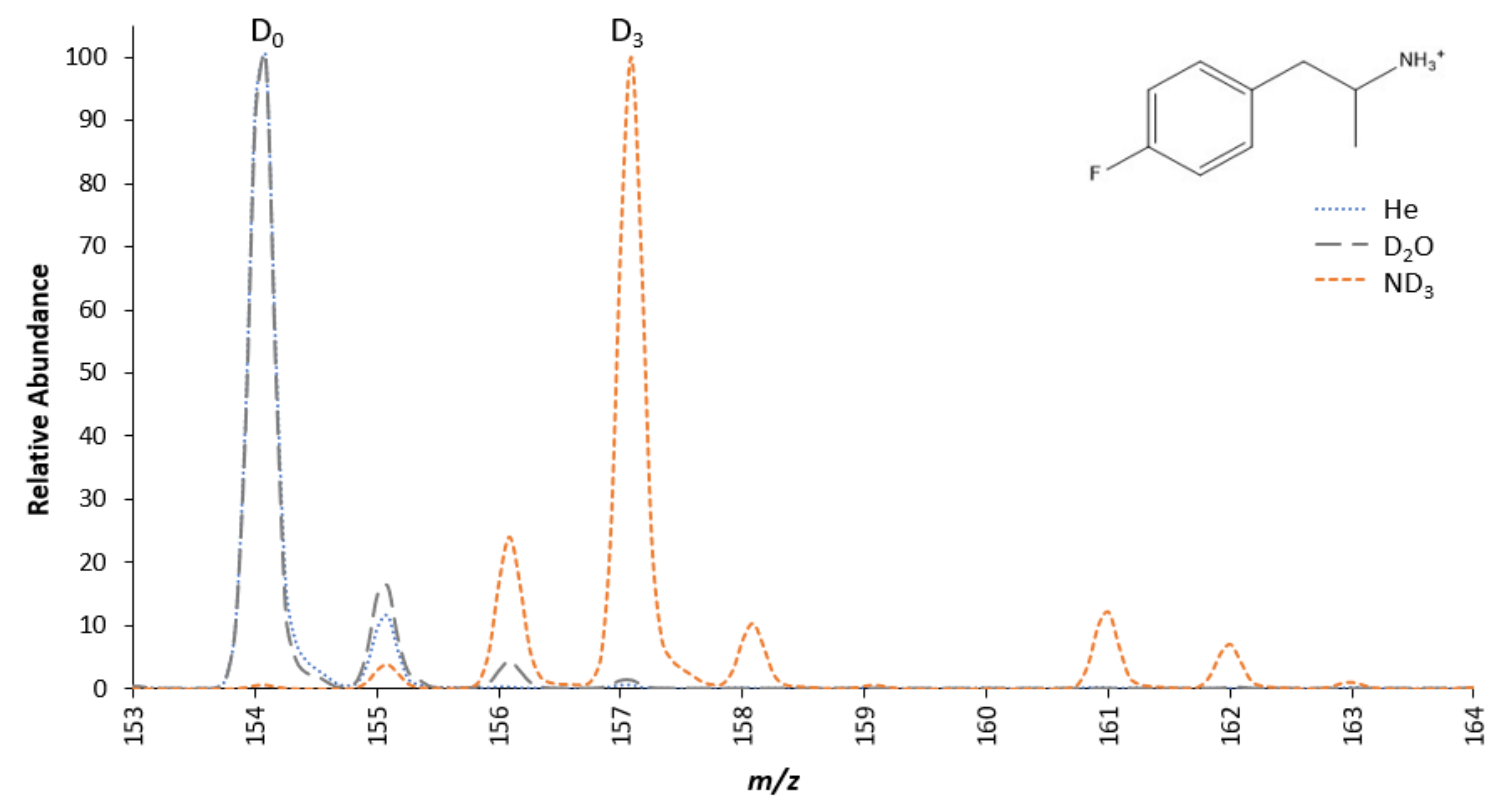

Figure C2b. Isotopic distribution for 4-fluoroamphetamine.

There is no deuterium uptake with $\mathrm{D}_{2} \mathrm{O}$, but a majority of ions exchanged 3 hydrogens in the presence of $\mathrm{ND}_{3}$.

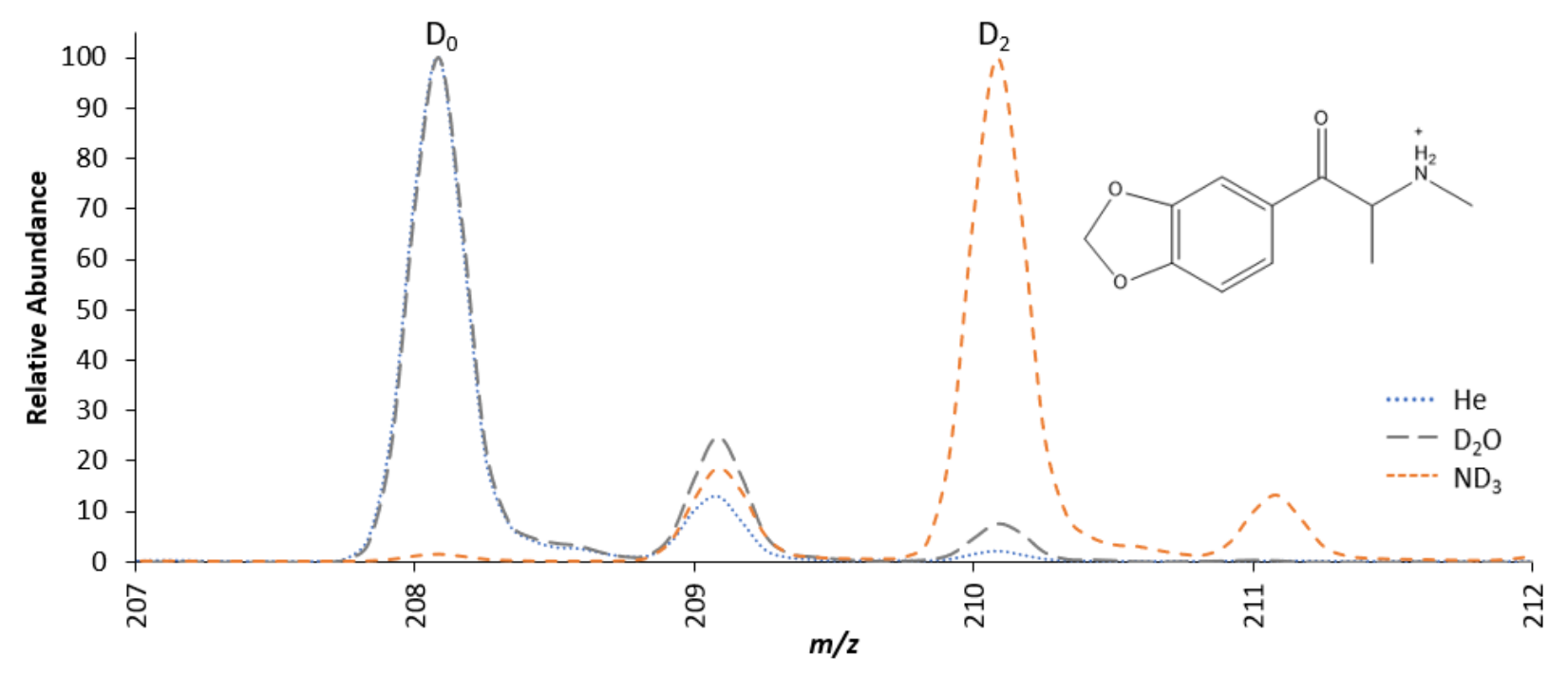

Figure C2c. Isotopic distributions for methylone.

There is minimal deuterium uptake with $\mathrm{D}_{2} \mathrm{O}$, but a majority of ions exchanged 2 hydrogens in the presence of $\mathrm{ND}_{3}$. 


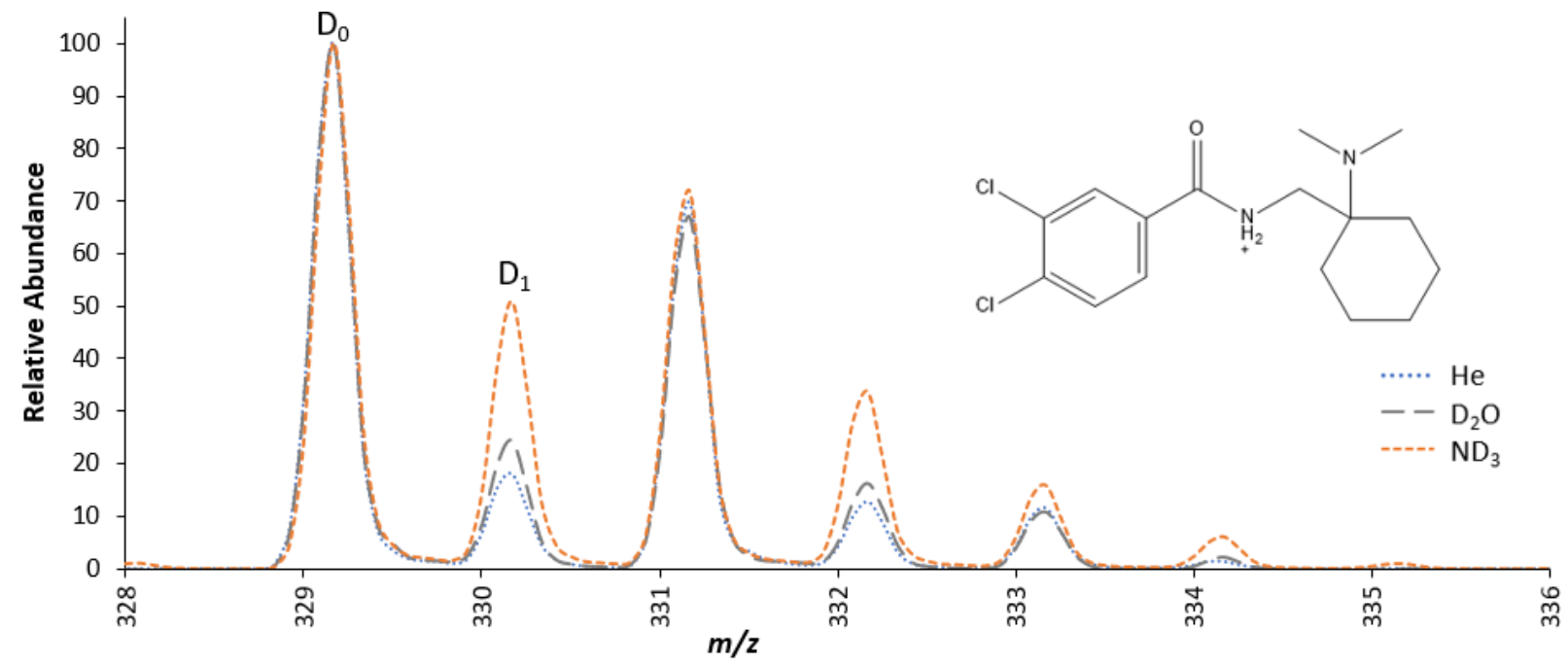

Figure C2d. Isotopic distributions for AH-7921.

There is no deuterium uptake in the presence of $\mathrm{D}_{2} \mathrm{O}$ and minimal deuterium uptake in the presence of $\mathrm{ND}_{3}$, as evidenced by the increased $m / z, 330$ abundance. The $\mathrm{m} / \mathrm{z} 133$ is the chlorine isotopic peak and is not the result of deuterium uptake.

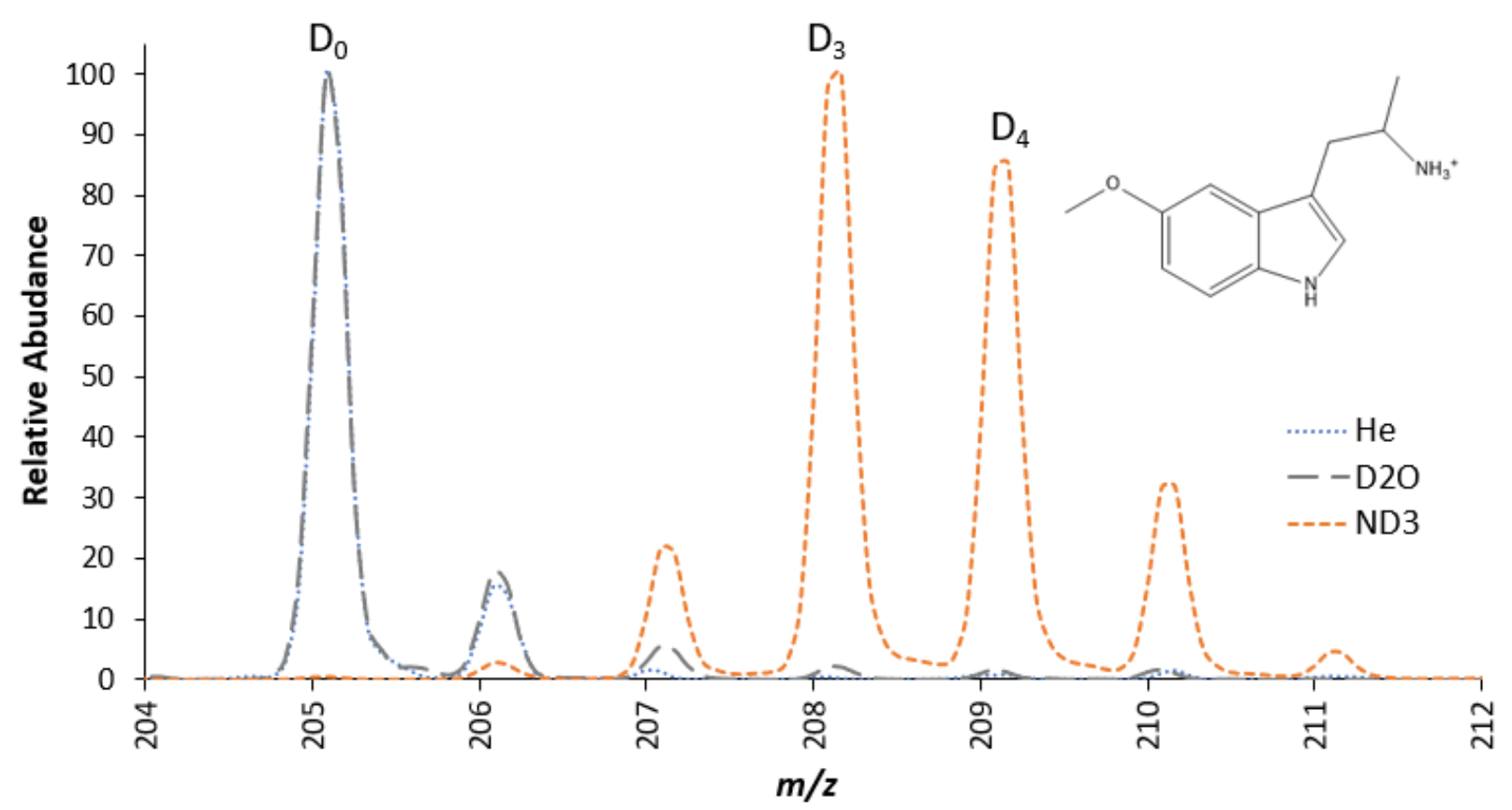

Figure C2e. Isotopic distributions for 5-MeO-AMT.

For reference, the $\mathrm{D}_{0}, \mathrm{D}_{3}$, and $\mathrm{D}_{4}$ isotopologue peaks are labeled and the protonated molecular structures are provided as insets. 


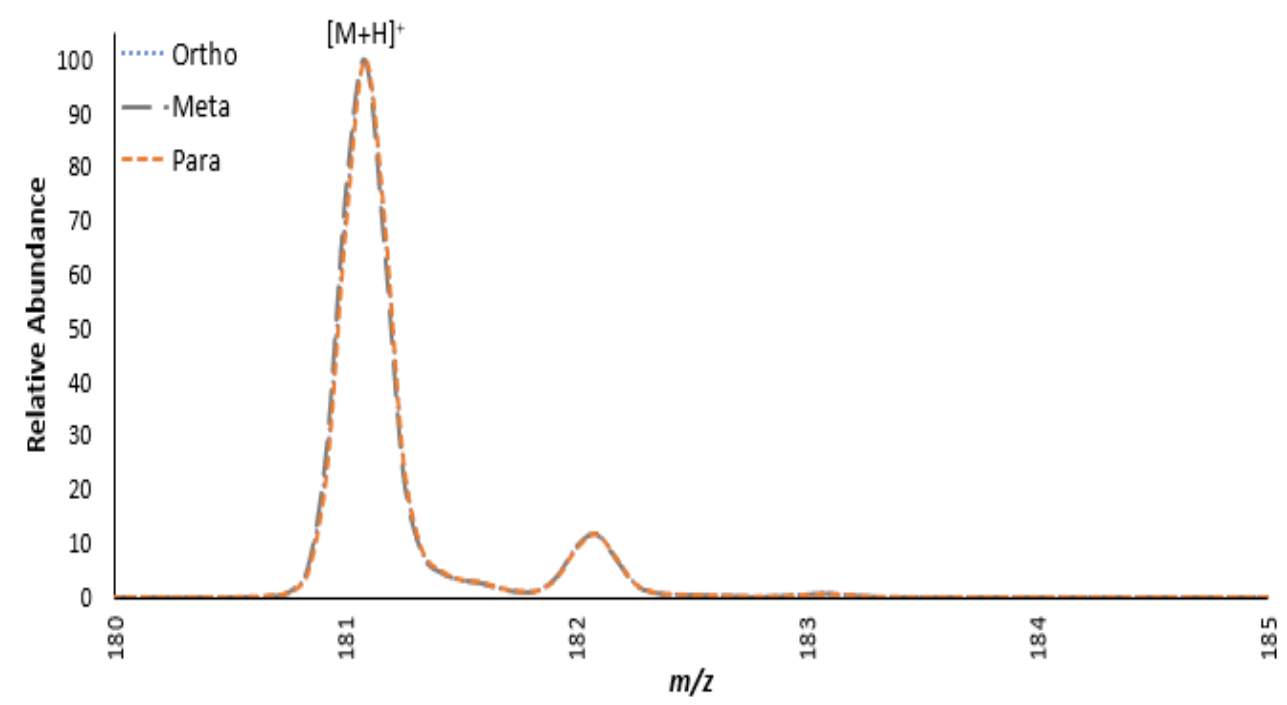

Figure C3. Isotopic distributions for all fluorophenyl piperazine isomers in buffer gas.

No reagent gas is present.

Table C2. Theoretical CCS mean and 95\% confidence interval range and dipole moments for the three fluorophenyl piperazine isomers.

These values are in good agreement with experimental CCSs reported. Theoretical dipole moments calculated for all saved coordinates in the first simulation for each isomer.

\begin{tabular}{|c|c|c|c|}
\hline \multirow[b]{2}{*}{ Isomer } & \multicolumn{2}{|c|}{ CCS } & \multirow{2}{*}{$\begin{array}{l}\text { Dipole Moment } \\
\text { Mean (Debye) }\end{array}$} \\
\hline & $\operatorname{Mean}\left(\dot{\mathrm{A}}^{2}\right)$ & 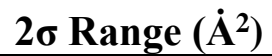 & \\
\hline Ortho & 77 & $74-80$ & 12.5 \\
\hline Meta & 78 & $75-81$ & 15.1 \\
\hline Para & 78 & $75-81$ & 16.0 \\
\hline
\end{tabular}




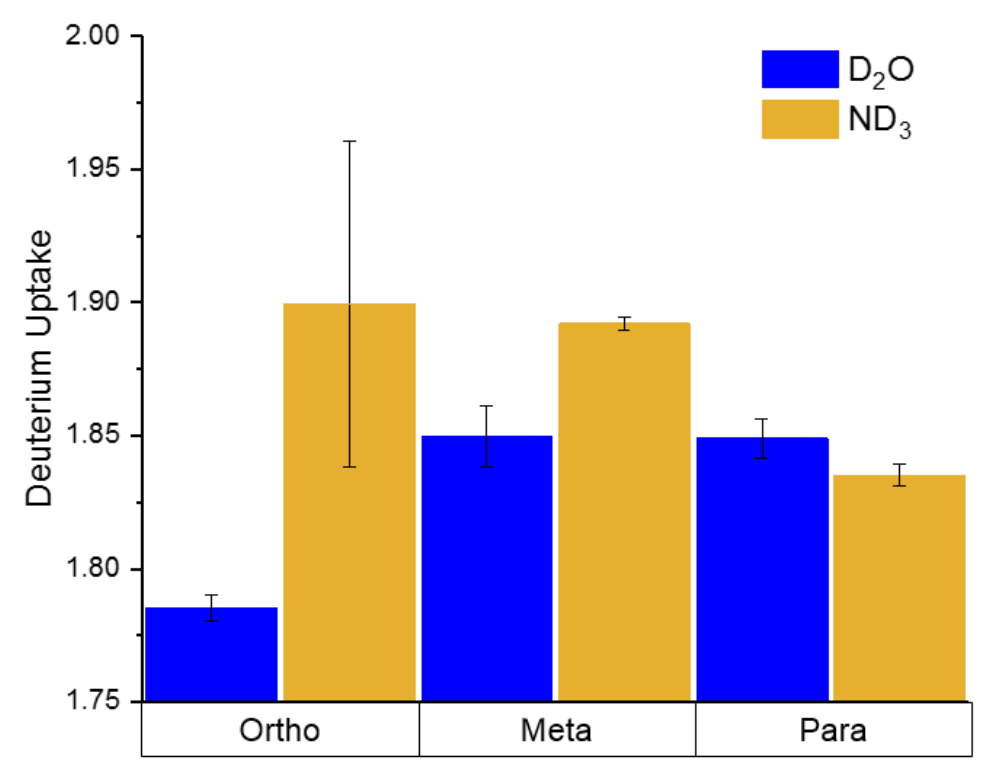

Figure C4. Comparison of deuterium uptake for the fluorophenyl piperazine isomers. Error bars are two standard deviations.

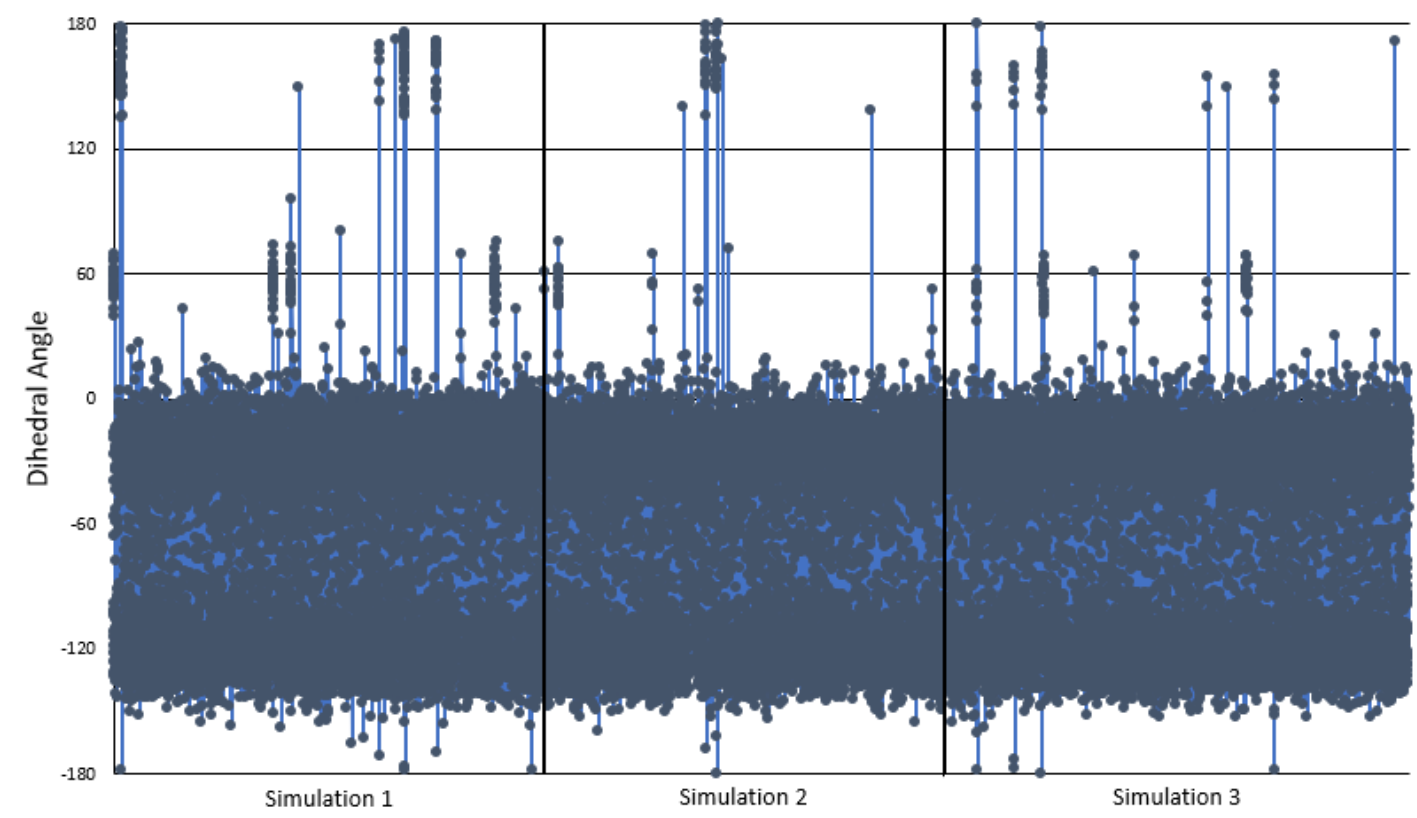

Figure C5a. Dihedral angle between the aromatic ring and the diamine ring for orthofluorophenyl piperazine.

Simulation triplicates shown. $\mathrm{X}$-axis is time, where each simulation starts at $0 \mathrm{~ns}$ and ends at 20 ns. 


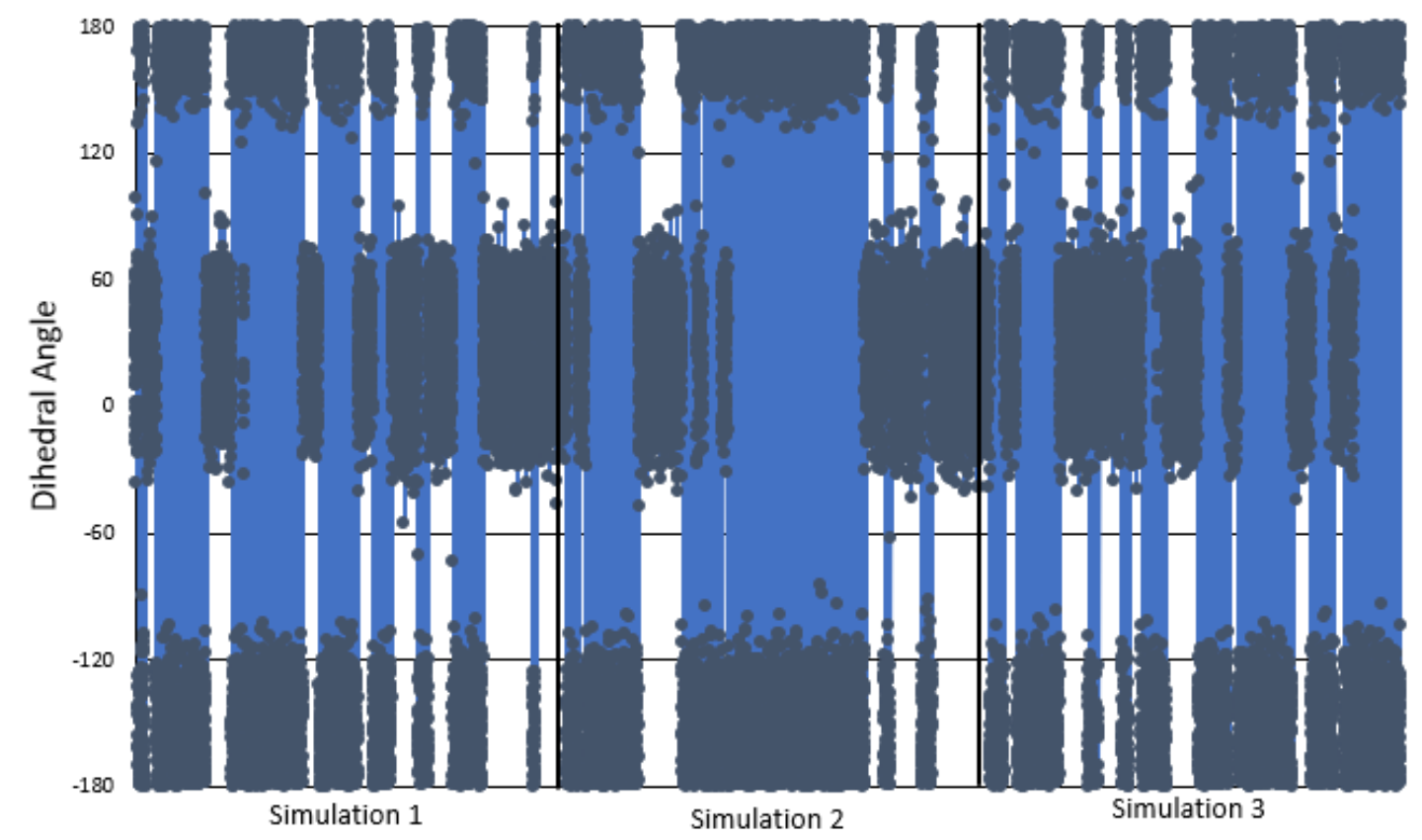

Figure C5b. Dihedral angle between the aromatic ring and the diamine ring for metafluorophenyl piperazine.

Simulation triplicates shown. $\mathrm{X}$-axis is time, where each simulation starts at $0 \mathrm{~ns}$ and ends at 20 ns.

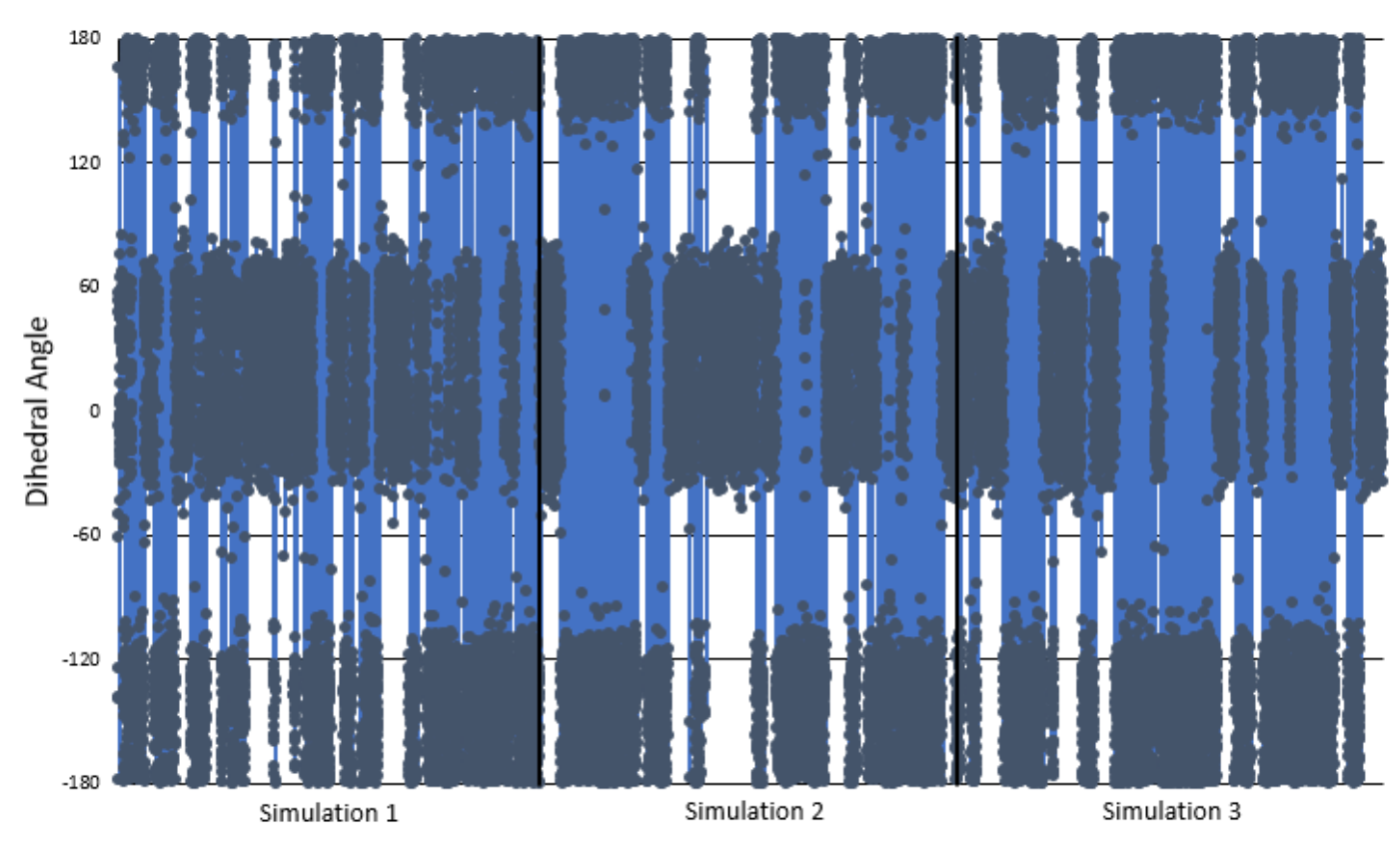

Figure C5a. Dihedral angle between the aromatic ring and the diamine ring for parafluorophenyl piperazine.

Simulation triplicates shown. $\mathrm{X}$-axis is time, where each simulation starts at $0 \mathrm{~ns}$ and ends at 20 ns. 